\title{
ABSTRACTS
}

\section{CGS 2020 \\ Book of Abstracts}

https://doi.org/10.5770/cgj.23.471

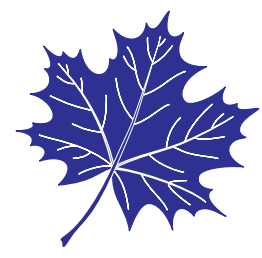

\section{FINALISTS FOR THE WILLARD AND PHOEBE THOMPSON AWARD}

\section{Understanding Geriatrician Practices in the Assessment and Management of Unintentional Weight Loss in Older Adults and the Use of Ice Cream to Address It \\ M. Gyenes ${ }^{1}$, S. K. Sinha ${ }^{2} .{ }^{1}$ Royal College of Surgeons in Ireland; ${ }^{2}$ University of Toronto.}

Background: Unintentional weight loss (UIWL) is common among older adults, but with few standards for its diagnosis and management. We conducted a global survey to examine how geriatricians screen for, diagnose, and manage UIWL, and their opinions around the use of ice cream to address it.

Methods: An online English language survey was developed and field-tested before geriatricians internationally were invited to complete it over a 16 -week period. Respondents were asked for demographic information, use of screening tools, diagnostic investigations, and use of pharmacological and non-pharmacological approaches to address UIWL, including recommending ice cream.

Results: 1131 geriatricians across 51 countries participated. $89.1 \%$ reported that they frequently see UIWL and that their most common Methods: used to evaluate it were performing a comprehensive history and physical examination (97.4\%) and assessing for cognitive impairment (86.5\%). $74.2 \%$ noted that they routinely prescribed oral nutritional supplements (ONS) and involved non-medical professional(s) (71.6\%) to manage UIWL. 50.4\% reported recommending ice cream to their patients with UIWL, although only $30.6 \%$ reported being aware of other colleagues doing so. Geriatricians practicing for $30+$ years were significantly more likely to recommend ice cream $(\mathrm{P}<0.05)$. A thematic analysis of free-text responses identified that prescribing ice cream supported patient preferences and socio-economic realities.

\section{Discussion: N/A}

Conclusion: UIWL is commonly seen amongst geriatricians worldwide. While a majority of the geriatricians surveyed routinely prescribe ONS and involve others to help manage
UIWL, at least half are also recommending ice cream to address it. This is the first study to examine geriatrician practices around managing UIWL. A practice amongst experienced geriatricians, the use of ice cream could be acknowledged and supported as a practical and cost-effective way to address UIWL that may better align with patient preferences.

\section{Quality Improvement Outcomes from the Introduction of a Formalized Geriatrician Presence in a Rehabilitation Setting}

S. Fitzgerald ${ }^{1}$, S. Sinha ${ }^{2}$, L. Romanovsky ${ }^{3} .{ }^{1}$ University of Toronto Faculty of Medicine; ${ }^{2}$ Mount Sinai Hospital and University Health Network; ${ }^{3}$ Mount Sinai Hospital.

Background: Currently, no known objective evidence exists to quantify the impact that Geriatricians have on patient and system outcomes when formally working in a rehabilitation setting. We studied the effect of adding the services of a geriatric medicine consultation in a geriatric-focused rehabilitation setting providing care to dialysis and non-dialysis patients.

Methods: A retrospective observational cohort study was conducted at an inpatient rehabilitation centre in Toronto, Ontario. Two geriatric groups were studied: rehabilitation $(\bar{X}=79.9$ years, $n=1330,51.9 \%$ female $)$ and dialysis patients $(\bar{X}=72.7$ years, $n=823,42.9 \%$ female). We analyzed data from a comprehensive geriatric assessment (CGA) exposure of all consecutively admitted patients between January 2009-June 2019. The primary outcome of interest was a change in the Functional Independence Measure (FIM) score before and after implementation of the formalized consult service and ongoing management. Secondary outcomes of interest included reduced numbers of service interruptions and reduced lengths of stay. QI Macros Software was used to construct statistical process control charts to analyse the data, in addition to descriptive statistics.

Results: X Bar-S Charts demonstrated a 96\% improvement in FIM Efficiency in both groups when comparing pre and post exposure. Similarly, the number of service interruptions was significantly reduced in both groups: $43.1 \%$ in geri-rehab and $57.1 \%$ in geri-dialysis. Length of stay was reduced by $10.6(28.1 \%)$ and $10.8(24.9 \%)$ days in geri-rehab and geridialysis, respectively. 
Discussion: This is the first study to objectively demonstrate that a geriatric consult service and ongoing management can uniquely improve quality-related patient and system outcome measures.

Conclusion: Introduction of comprehensive geriatric assessment among geriatric rehabilitation inpatients resulted in significant improvement in FIM Efficiency, and reductions in the number of service interruptions and lengths of stay.

\section{Managing Incontinence -A Community Based Participatory Research Approach}

\section{Kirillovich, S. Rajabali, A. Wagg. University of Alberta.}

Background: Urinary incontinence (UI), the complaint of involuntary leakage of urine, has a substantial impact on the quality of life of older adults. Most UI research is driven by researchers and lacks the patient perspective. The goal of this study was to gain the perspective of older adults in formulating a research agenda, tailored to address their questions and improve their experience with managing UI.

Methods: Using community based participatory research Methods: , an advisory group of eight older adults with UI was recruited to be on the research team. An initial focus group was conducted to learn about their research needs. Nominal Group Technique was used and data was analyzed thematically. Employing the Delphi consensus method, an online questionnaire, containing 20 priorities for future UI research and education was developed. The online questionnaire was advertised across Canada.

Results: Fifty nine older adults with UI rated each priority on a Likert scale in the first round. The second round received an $85 \%$ response rate. A total of 11 priorities of $>80 \%$ agreement were retained. The highest rated priorities included relationship between physical activity and UI, support for those with UI, causes of UI and its management, public restroom accessibility and sleep and UI.

Discussion: The highest rated priorities, while identifying the research needs of older adults with UI, also reflected a lack of knowledge amongst older adults regarding UI despite extensive research available on these topics. This may be due to lack of effective knowledge translation strategies through a media that is accessible to older adults.

Conclusion: Involving older adults through CBPR is a practical method to gain their perspective and help researchers and health care professionals formulate a research and education agenda to address the concerns of older adults with UI.

\section{Assessing Geriatric Trauma Patient Outcomes and the Effect of Antithrombotic Medications}

A. Morgan ${ }^{1}$, N. Beauregard ${ }^{2}$, E. Saidenberg ${ }^{3}$, J. Lampron ${ }^{3}$.

${ }^{1}$ University of Ottawa; ${ }^{2}$ Ottawa Hospital Research

Institute; ${ }^{3}$ The Ottawa Hospital.
Background: to assess the outcomes of geriatric (over 60 years old) trauma patients and determine the impact of antithrombotic medications on these patients.

Methods: We conducted a retrospective review collecting demographic and outcome data from a prospectively maintained level 1 trauma centre registry from January 2017 to December 2018. Descriptive statistics were used to evaluate and compare outcomes.

Results: Of the 1616 patients included in this study, 745 patients were over 60 years old. Older patients had higher mean injury severity scores (ISS, 17.9 \pm 8.0 ) and longer hospital stays (12.9 \pm 15.9 days) than younger patients $(\mathrm{p}=0.04$ and $\mathrm{p}=0.008$, respectively). Older patients overall were less likely to receive surgery or angioembolization, tranexamic acid (TXA), and blood products than younger patients $(\mathrm{p}<0.001)$. Despite similar ISS, older patients taking antithrombotic medications were less likely to receive surgery and/or angioembolization or be discharged home than those not taking antithrombotic medications ( $p=0.03$ and $p=0.02$, respectively). In-hospital mortality rates increased with age, but rates between older patients on antithrombotic therapy and those who were not.

Discussion: The study showed that, in general, older trauma patients experience worse outcomes and receive different treatment than younger trauma patients. Given the known benefits of anti-fibrinolytic therapy in traumatic bleeding, it is surprising that older patients were less likely to receive TXA despite higher ISS. Interestingly, there was no difference in mortality for geriatric patients taking antithrombotic therapy.

Conclusion: Older adult trauma patients received fewer surgical interventions, anti-fibrinolytics, and transfusions than younger patients and had worse outcomes overall. Further research exploring the factors driving the differences identified in this study is necessary for improving geriatric patient care.

\section{Is Opioid Agonist Therapy Age Friendly? A Systematic Review of Older Adult Experience}

M. Wylie, L. Nixon, K. A. Hayden. University of Calgary.

Background: Older adults with an opioid use disorder (OUD) are growing in number. Meanwhile, opioid agonist therapy (OAT) services have largely been developed with the younger population in mind, leaving a gap in treatment programs tailored to the needs of older adults. The current systematic review aims to gain an understanding of older adult (50+) service user experiences during OAT. This review is underway to 1) Examine current OAT service delivery compatibility for older adults by considering patient experience from the literature using a sensitizing age-friendly service framework with aim to 2) Inform policy and program changes to improve OAT service delivery for older adults.

Methods: Systematic search focusing on three concepts: 1) opioid agonist therapy (e.g. methadone and 
buprenorphine-naloxone), 2) older adults, and 3) patient experiences. Six databases were searched. Articles screened based on title and abstract; selected full text articles screened by two reviewers independently. Framework synthesis analysis of included studies is underway. Joanna Briggs Institute Appraisal Tools guided quality assessment.

Results: 3235 articles screened (title/abstract), 72 reviewed (full text). Preliminary findings include barriers related to agefriendly domains: respect and social inclusion, social isolation, and community support and health services. Barriers will be synthesized across identified literature to generate program change recommendations.

Discussion: Negative experiences exist for older adults in OAT relating to program-required direct-observed/daily dosing, stable housing, and opioid abstinence. Older adults in OAT experience multiple intersecting but distinct stigmas having to do with: older age, drug use, and OAT itself.

Conclusion: OAT is an evidence-based and potentially lifesaving treatment for people with OUD. Early analysis suggests delivery of OAT services is not sensitive to the needs of older adults and can be expected to contribute to inequitable and negative health outcomes.

\section{FINALISTS FOR THE RÉJEAN HÉBERT CANADIAN INSTITUTES OF HEALTH RESEARCH-INSTITUTE ON AGING PRIZE}

\section{The Role of Medical Assistance in Dying in Patients with Dementia: Assessing the Attitudes of Clinicians in Vancouver, British Columbia Through Analysis of Quantitative and Qualitative Survey Data \\ L. Cuthbertson ${ }^{1}$, A. Nakanishi ${ }^{1}$, J. Chase ${ }^{2} .{ }^{1}$ University of British Columbia; ${ }^{2}$ Providence Health Care, Division of Geriatric Medicine.}

Background: Medical Assistance in Dying (MAiD) became legal in Canada in June 2016. Current legislation requires people to have the capacity to confirm their consent immediately prior to the procedure. As such, many Canadians living with dementia are excluded from accessing MAiD. In January 2020, the Government of Canada launched public consultation to give Canadians the opportunity to provide their views on changes to the legislation that would include advanced requests for MAiD. This study explores the ethical and medical concerns held by healthcare providers related to MAiD and dementia.

Methods: Vancouver clinicians, including residents, physicians and nurse practitioners who care for older adults, completed an online survey using the UBC Qualtrics platform.
The survey included questions assessing knowledge and attitudes about MAiD in dementia, as well as four fictional clinical vignettes designed to solicit participant reasoning around access to MAiD for persons with dementia, including advance requests. Quantitative data was graded on a Likert scale, and narrative responses were coded and analyzed by two independent researchers using thematic analysis. The project was approved by the UBC Behavioural Research Ethics Board.

Results: Of 87 survey respondents, 73 (85.9\%) were practicing physicians or nurse practitioners and 12 (14.1\%) were trainees. Several themes were identified from participant responses to the four fictional clinical vignettes, including patient autonomy, interests of the future self with dementia, intolerable suffering, and potential for abuse or secondary gain.

Discussion: This study highlights that attitudes among clinicians regarding the role of MAiD in persons with dementia are diverse. Specific concerns and potential solutions were identified through participant responses.

Conclusion: This study provides valuable clinician input relating to possible legislative changes for advanced requests for MAiD.

\section{Association Between Medications with Anticholinergic Properties and Falls in Hospitalized Older Adults}

\section{P. Horsley ${ }^{1}$, A. Huang ${ }^{2} .{ }^{1}$ University of Ottawa; ${ }^{2}$ The Ottawa} Hospital, Division of Geriatric Medicine.

Background: Falls in older adults have been associated with longer hospital stay and time required for rehabilitation, worse disability and quality of life, and increased risk of death. Studies have shown that community-dwelling older people who use medications with anticholinergic properties have an increased risk of falling. We studied the association between the use of medications with anticholinergic properties and falls in hospitalized older adults.

Methods: Observational case-control study. Data were obtained from the electronic health records of an academic health center concerning all people 65-years and older admitted during 2015, excluding ICU, mental health and gynecology services. All inpatient prescriptions, medication administration records (MARs) and fall events were available. A convenience sample of 42 fallers were matched to controls by age, sex, admission date and service. Medication exposure was confirmed by the MARs. The anticholinergic property of each drug administered was classified using the AntiCholinergic Burden (ACB) scale and a cumulative score was calculated for the day of and one day preceding a person's fall event. Analyses were done using SAS.

Results: 19,425 admissions to hospital and 896 fall events were recorded. The mean (SD) ACB score for fallers was 
2.55(1.90) compared to $1.79(1.65)$ for controls. The odds ratio $(95 \% \mathrm{CI})$ was $1.50(1.07,2.11 ; \mathrm{p}=0.020)$ for cumulative $\mathrm{ACB}$ score on the day before the index fall and $1.39(1.00,1.92$; $p=0.048$ ) on the day of the index fall.

Discussion: Fallers were exposed to a higher ACB load than controls and the time of exposure did not significantly alter the effect.

Conclusion: Medications with anticholinergic properties have a significant effect on fall risk in hospitalized older adults. Further study needs to be done with a larger sample to validate this finding.

\section{Adverse Events in ALC Patients Awaiting Long-Term Care in Hospital}

\section{G. L. Fat, A. Gopaul, M. M. Taabazuing. Western University.}

Background: A rising number of elderly patients are awaiting long-term care (LTC) placement in hospital. These patients are designated Alternate-Level-of-Care (ALC) to indicate lack of acute issues. In addition to the infrastructural strain this creates by occupying beds allocated for acute care, it is hypothesized that ALC patients incur a high number of healthcare-associated adverse events during their prolonged hospitalization. In our retrospective cohort study, we describe rates of healthcare associated adverse events in ALC patients awaiting LTC in hospital and compare to patients already in LTC.

Methods: Our chart review includes a random sample of 165 patients of 2386 who were designated ALC awaiting LTC between January 2015 and December 2018 at Victoria Hospital and University Hospital, two tertiary care centres in London, Ontario. Charts are reviewed to determine incidence of adverse events, which include infections, falls, delirium, pressure ulcers, and venothrombotic events. Events are recorded over the course of the patients' ALC designation, until LTC placement or death while awaiting LTC.

Results: Data collection is ongoing and will be completed for the ALC cohort by March 2019. We will present the rates of adverse events in this cohort, describing their relative prevalence and relationship with length of stay. Preliminary Results: : indicate a positive correlation between length of stay and the incidence of adverse events, with falls and delirium being most common.

Discussion: Pending analysis of full results, our discussion will hypothesize how the ALC cohort compares to similar patients who await LTC at home, and patients already in LTC. We will also comment on the financial impact of ALC patients in hospital and prospective strategies to reduce ALC stays.

Conclusion: Conclusion and updated abstract pending data collection and analysis (March 2019).

\section{A Canadian Environmental Scan of Perioperative Geriatric Models of Care}

B. Cheung I, S. Chan' ${ }^{2}$ D. McIsaac ${ }^{2}$, R. Khadaroo ${ }^{3}$, C. Wong ${ }^{l} .{ }^{1}$ University of Toronto; ${ }^{2}$ University of Ottawa;

${ }^{3}$ University of Alberta.

Background: Despite evidence that specialized perioperative care improves outcomes of older patients, there is little data on perioperative geriatric care models in Canada. This study describes the state of perioperative geriatric services in Canadian hospitals.

Methods: Questionnaire items were adapted from similar surveys conducted in Australasia and United Kingdom. Sensibility testing was performed by reviewers from geriatrics, anesthesiology, and surgery for content validity. The survey was iteratively constructed and pilot-tested 2 weeks apart for test-retest reliability. The web-based survey was disseminated via mailing lists, public domain emails, and Twitter to Canadian geriatricians, anesthesiologists, and general surgeons from September-December 2019.

Results: There were 132 respondents (78\% geriatricians, $11 \%$ general surgeons, $7 \%$ anesthesiologists, $4 \%$ other) representing 85 hospitals across all provinces. 40 of 85 hospitals $(50 \%$ community, $50 \%$ academic) have a perioperative geriatric program with 15 having pre-operative, 5 intra-operative, and 30 post-operative services. Program eligibility is primarily based on age and procedure type. Pre-operative risk is determined by comprehensive geriatric assessment (15\%), cognitive screening $(12 \%)$, and frailty screening (11\%). Pre-operative services include delirium counselling (17\%), medication review (17\%), and geriatrics consultation (13\%). Post-operative services include geriatric-specific order sets (22\%), geriatric-surgery co-management models (19\%), and geriatrics consultation (18\%). Free-text comments highlighted the need and limited resources for perioperative geriatric care.

Discussion: Recognizing the survey results are likely overestimated due to reporting bias, this study demonstrates low incorporation of perioperative geriatric care in Canadian hospitals. Most programs focus on post-operative care and share similar eligibility criteria, pre-operative risk assessments, and services.

Conclusion: This study provides a national overview of perioperative geriatric programs in Canada and suggests a need to understand barriers to perioperative geriatric care to support effective spread of this evidence-based care.

\section{Trends in Synthetic Cannabinoid Prescriptions Among Older Adults in Ontario, Canada, from 1997-2017}

D. Sommer' , N. M. Stall ${ }^{2}$, V. Giannakeas ${ }^{3}$, J. S. Zipursky ${ }^{4}$, J. A. Watt ${ }^{5}$, P. A. Rochon ${ }^{6}{ }^{1}$ University of Toronto; ${ }^{2}$ University of Toronto, Institute of Health Policy, Management and 
Evaluation; University of Toronto, Women's College Research Institute; Division of General Internal Medicine and Geriatrics, Sinai Health System; ${ }^{3}$ Women's College Research Institute; ICES, Toronto; ${ }^{4}$ University of Toronto, Institute of Health Policy, Management and Evaluation; University of Toronto, Division of General Internal Medicine and Geriatrics, Sinai Health System; Sunnybrook Health Sciences Centre; ${ }^{5}$ University of Toronto; St. Michael's Hospital; ${ }^{6}$ University of Toronto, Institute of Health Policy, Management and Evaluation; University of Toronto, Women's College Research Institute, Women's College Hospital; ICES, Toronto.

Background: Despite limited evidence, synthetic cannabinoids are prescribed to older adults for a variety of medical conditions including chronic pain, sleep disturbances, and the behavioural and psychological symptoms of dementia. In Ontario, nabilone accounts for $>98 \%$ of all cannabinoid prescriptions.

Methods: We conducted a population-based, cross-sectional analysis using administrative health databases held at ICES to examine the yearly incidence and prevalence of nabilone prescriptions to persons $\geq 66$ years of age in Ontario, Canada between January 1, 1997 and December 31, 2017. We also described the characteristics of older adults who filled a prescription for nabilone in 2017. We defined "on-label" nabilone use as a prescription filled within 30 days of chemotherapy.

Results: The rate of Nabilone prescription per 1,000,000 older Ontarians increased from 25.8 users in 1997 to 864.4 users in 2007, while further increasing to 3,363.6 users in 2017. In $2017,92.8 \%$ of prescriptions were "off-label" and $12.4 \%$ occurred in nursing homes. Older adults prescribed nabilone had a median of four comorbidities, and $16.2 \%$ had dementia. In those prescribed nabilone, $49.1 \%, 20.8 \%$, and $11.4 \%$ were concurrently prescribed antidepressants, sedatives, and antipsychotics, respectively.

Discussion: There have been substantial 20-year increases in nabilone prescribing to older Ontarians, including a 3.7-fold increase over the last 5 years. The majority of prescriptions were "off-label", and to individuals with multiple comorbidities taking other psychoactive medications. Despite limited evidence for clinical benefit and largely unknown harms, providers may be prescribing nabilone due to increased societal acceptance of cannabis, and because few safe and effective alternatives exist for chronic conditions commonly affecting older adults.

Conclusion: Further research is needed to characterize potential harms associated with observed increases in "off-label" nabilone prescribing among older adults.

\section{Impact of Dementia on Outcomes in a Seniors Falls Prevention Program}

L. Schnarr, M. Bater. Royal Jubilee Hospital, University of British Columbia.
Background: Elderly individuals with dementia have a significantly higher falls risk than those with normal cognition. Studies have demonstrated the effectiveness of exercise for falls prevention. The purpose of this study was to analyze data on participants in a falls prevention program to determine if dementia impacted intervention efficacy.

Methods: Retrospective chart review analyzing pre-and post-intervention data was performed for participants in the Falls Prevention Program at Royal Jubilee Hospital (Victoria, $\mathrm{BC})$. Subjects $(\mathrm{n}=167)$ had a documented MOCA score and pre-and post-program scores for at least one of five tests (Berg Balance Scale, gait velocity preferred, gait velocity fast, timed up-and-go, 30-second sit-to-stand). Paired t-tests and ANOVA tests were used to analyze pre-and post-scores based on MOCA result.

Results: Participants without dementia $(\mathrm{MOCA}=26+$ ) demonstrated improvement in scores for all 5 tests $(\mathrm{p}<0.001)$. Those with mild dementia $(\mathrm{MOCA}=18-25)$ improved in all except for gait velocity preferred $(\mathrm{p}<0.05)$. The moderate dementia group (MOCA=10-17) only showed improvement for Berg Balance Scale $(p<0.001)$. No participants had severe dementia $(\mathrm{MOCA}<10)$. When comparing the interval change in scores between those with dementia (MOCA $<26)$ and those without $(\mathrm{MOCA}=26+)$ there was only a significant difference identified in timed up-and-go scores $(\mathrm{p}<0.05)$.

Discussion: Scores on tests indicative of falls risk and frailty improved after participation in a falls prevention program for those with mild dementia but not moderate dementia, demonstrating benefit in the mild group. No difference in improvement of scores was seen when comparing those with normal cognition versus any severity of dementia, leading us to believe that these two groups derived similar benefit from the program.

Conclusion: Falls prevention programs appear to be effective in those with mild dementia and should be used to try and reduce morbidity and mortality.

\section{FINALISTS FOR THE JACK MACDONELL PRIZE}

\section{Addressing Loneliness in Older Adults as Part of the Comprehensive Geriatric Assessment}

A. Miles, P. Jung, C. Reppas-Rindlisbacher, R. Rofaiel, V. Y.Y. Xu, D. Gandell, A. Steiman, S. Veinish, M. Norris. University of Toronto.

Background: Loneliness in older adults is associated with adverse health outcomes including functional decline, frailty, cognitive impairment, and death. Despite the prevalence, impact, and potential for intervention, loneliness in older adults is under-recognized. We aimed to increase physicianled awareness and identification of loneliness in geriatric medicine outpatient clinics. 
Methods: Quality improvement methodology was used. We hypothesized that loneliness is under-recognized in academic geriatric medicine outpatient clinics, with the root cause being limited physician awareness. In line with this theory, we introduced a validated tool that served to standardize loneliness identification. The screening tool was integrated into outpatient Comprehensive Geriatric Assessment (CGA) forms in subspecialty resident clinics. The primary outcome measure was the proportion of patients who screened positive for loneliness that received a loneliness intervention. Early change cycles focused on process measures evaluating implementation of the screening tool, while later cycles furthered our understanding of loneliness in geriatric medicine practice.

Results: Gap analysis revealed less than $10 \%$ of new patients presenting to four geriatric subspecialty resident clinics had been screened for loneliness. Introduction of the screening tool increased screening for loneliness to $75 \%$ of new patients receiving a CGA. Loneliness interventions were discussed with all patients who screened positive for loneliness, but patients frequently declined interventions.

Discussion: Loneliness is a complex and heterogenous issue. While screening was easily implemented, designing interventions required an individualized approach and was outside the scope of a CGA. Future change cycles will include engaging other professions in developing loneliness interventions and assessment of loneliness in caregivers.

Conclusion: Including a short loneliness screen in the CGA led to increased identification of lonely patients and initiation of interventions toward mitigating loneliness in older adults.

\section{Comparison of eConsult Medication Histories from Referring Clinicians Compared to Pharmacist-Acquired BPMHs: A QI Study}

\section{R. Rofaiel ${ }^{1}$, J. Ho ${ }^{2}$, B. Liu ${ }^{l}$, E. To ${ }^{2}$, J. Bodkin ${ }^{2}{ }^{1}$ University of Toronto; ${ }^{2}$ McMaster University.}

Background: Complete medication lists are necessary for medication optimization. Specialist use of eConsults, an increasingly used mode of telemedicine to provide clinical recommendations, relies on the medication list provided by the referring clinician. The accuracy of medication lists provided by referring clinicians at the time of an eConsult is unknown. We sought to characterize medication list discrepancies between those provided by referring clinicians using eConsults compared to those obtained by a pharmacist-led best possible medication history (BPMH).

Methods: We conducted a retrospective chart review of older community-dwelling patients who received a GeriMedRisk eConsult (provincial geriatric clinical pharmacology and psychiatry program in Ontario, Canada) between June 1, 2018 to July 1,2019 . Other inclusion criteria were age $>=65$ years, $>=3$ medications, and having a pharmacist-led BPMH. The primary outcome was the incidence of medication discrepancies. We also characterized the severity and clinical relevance of each medication discrepancy.

Results: Of the 42 patients who met inclusion criteria, 40 patients had at least one discrepancy (95\%) with an average of 6.2 discrepancies per patient (Standard deviation 5.2). Thirty-two patients (76.2\%) had omissions, $25(59.5 \%)$ had commission, 20 (47.6\%) with incorrect dose and 17 (40.4\%) had an incorrect frequency discrepancy. Twenty-eight (70\%) patients had discrepancies included on the Beers criteria with $33.9 \%$ identified as clinically significant. Medications most commonly associated with error included acetaminophen, Vitamin B12, salbutamol, hydromorphone and lorazepam.

Discussion: This small exploratory study found that pharmacist-led BPMHs identified medication discrepancies in the majority of older Ontario eConsult patients with polypharmacy. These errors had potential to cause harm or to impact clinical recommendations.

Conclusion: Physicians providing eConsults for older patients with polypharmacy should consider the accuracy of the medication lists provided.

\section{Sarcopenia Adds to Clinical Evaluation in Predicting Treatment Tolerability and Survival in Aggressive Non-Hodgkin B Cell Lymphoma}

M.-F. Forget ${ }^{1}$, I. Fluery ${ }^{2}$, L. Marchand ${ }^{3}$, S. Drory ${ }^{2}$, I. Ahmad ${ }^{2}$, H. T. Wang ${ }^{2}{ }^{1}$ Université de Montréal;

${ }^{2}$ Hôpital Maisonneuve-Rosemont; ${ }^{3}$ Centre Hospitalier de l'Université de Montréal.

Background: Sarcopenia is associated with decreased treatment tolerance and overall survival in diffuse large B-cell lymphoma, the most common non-Hodgkin lymphoma (NHL) which incidence is age related. The impact of sarcopenia on event-free survival (EFS) in older adults with an aggressive B-cell NHL has not been well studied. The purpose of this study was to evaluate the association between sarcopenia and 6-month EFS in adults aged 65 and above, diagnosed with an aggressive B-cell NHL.

Methods: We performed a retrospective cohort study of older adults diagnosed with an aggressive B-cell NHL between July 1, 2014, and June 30, 2018, and who completed first line chemotherapy. Sarcopenia was defined as the lowest tertile of psoas muscle index (PMI). Psoas muscle area was measured on a single axial slice at the level of L4, using CoreSlicer ${ }^{\circ}$ software, and divided by squared height. EFS, defined as no death, relapse or hospitalization, was compared between sarcopenic and non-sarcopenic using Kaplan-Meier curves and multivariable Cox Regression.

Results: In our 62 older adults' cohort, median age was 74 (69-78) and median PMI was $7.24 \mathrm{~cm}^{2} / \mathrm{m}^{2} .20$ patients were sarcopenic with a median PMI of $5.5(4.8-6.3) \mathrm{cm}^{2} / \mathrm{m}^{2}$. Patients 
received full dose chemotherapy (29,56\%) or reduced dose (33, $44 \%$ ). After controlling for age, sex, ECOG, cardiac disease, creatinine, LDH and hemoglobin levels, sarcopenia was associated with a lower EFS (HR 3.5, 95\%CI: 1.1-11, $\mathrm{p}=0.032$ ). When stratifying for dose of chemotherapy, there was a trend for a decreased EFS with sarcopenia in the full dose group $(p=0.095)$ but it did not reach statistical significance.

Discussion: Being sarcopenic is associated with a lower 6-month EFS in older adults who completed first line chemotherapy.

Conclusion: Sarcopenic older adults might benefit from prechemotherapy geriatric evaluation and tighter follow-ups.

\section{The Use of Prisma-7 Questionnaire Within the McGill University Health Centre}

D. Milroy ${ }^{1}$, E. Marrone ${ }^{1}$, M. Maalouf ${ }^{2}$, J. A. Morais ${ }^{3}$.

${ }^{1}$ Department of Family Medicine, McGill University;

${ }^{2}$ Quality Improvement Office, McGill University Health

Centre (MUHC); ${ }^{3}$ Division of Geriatric Medicine, McGill

University and McGill University Health Centre (MUHC).

Background: The PRISMA-7 is employed as a frailty screening tool in the McGill University Health Center (MUHC) adult hospitals. This study sought to assess its use in the MUHC and whether a positive PRISMA-7 score $(\geq 4)$ was associated with important hospital use characteristics.

Methods: A dataset was generated by querying MedUrge electronic medical record in the emergency department (ED) and patient registration databases at the MUHC for the 20172018 financial year for patients $\geq 75 \mathrm{y}$ old. Comparisons were made (OR; $\pm 95 \%$ CI) between those who scored $\geq 4$ vs. $<4$.

Results: The dataset comprised a total of $12331 \mathrm{ED}$ visits with 8226 unique patients. The PRISMA-7 was completed in $86 \%$ of ED visits and $45 \%$ tested positive (frail) with an increasing frequency with age. $52 \%$ of males tested positive, compared to $38 \%$ of females. In the ED, frail patients stayed longer than $48 \mathrm{~h}(1.43 ; 1.43-1.44)$, had increased geriatrician (1.94; $1.92-1.96)$ and specialized nurse consultations $(1.60 ; 1.57-$ $1.63)$, and were more likely to be admitted $(1.69 ; 1.69-1.69)$. Following admission, frail patients had increased likelihood of staying $>1$ week $(1.21 ; 1.20-1.22)$, of placement in long term care $(2.00 ; 1.98-2.01)$, and of returning to the ED within 30 days following discharge $(1.53 ; 1.52-1.54)$.

Discussion: While the PRISMA-7 cut-off of $\geq 4$ is different in the MUHC compared to $\geq 3$ in many studies, the incidence of frailty is consistent with the literature. More males were identified as frail and there was a peak incidence of frailty after 85 years of age due to specific questions within the PRISMA-7.

Conclusion: The association between a positive PRISMA-7 and important hospital use characteristics is consistent with the PRISMA-7's use as a frailty screening tool within the hospital ED setting.

\section{Prediction of Fall Risk Based on Underlying Cognitive Status: Results: from a Retrospective Analysis of the Canadian Longitudinal Study on Aging}

A. S. Halme, O. Beauchet. Division of Geriatrics,
Sir Mortimer B. Davis Jewish General Hospital.

Background: Falls are a public health concern as they are the leading cause of injury hospitalizations in seniors and cause significant morbidity and mortality. A strong association with neurocognitive disorders (NCD) has been established, which was not studied in Canada. Our objective was to model probability of falls in community-dwelling older Canadians from known risk factors, including cognition.

Methods: Risk of falls was determined from self-report in the previous year for participants over age 65 in a cross-section of the Canadian Longitudinal Study on Aging ( $\mathrm{n}>50000)$. The database provided detailed information on function, cognitive complaints, and neurocognitive and physical testing. Hence, we classified individuals as cognitively healthy, or with mild or major NCD. The cohort was randomly divided in training and validation subsets (7:3 ratio). In the former, risk of falls and of binarized recurrent falls were calculated using multivariate logistic regression (LR) with usual risk factors and cognitive diagnosis as predictors. We applied the results to the validation subset and computed $\mathrm{C}$-statistics.

Results: We had complete data for 9408 participants: $48.1 \%$ female with mean age $72.6 \pm 5.5$, in which $19.4 \%$ had $\geq 1$ fall in the previous year. LR showed the most significant risk factors for any fall were major NCD (OR 1.52 [1.04 - 2.21]), male sex (1.14 [1.02 - 1.27]) and living alone (OR 1.25 [1.11 $-1.39])$. The C-statistic was 0.59 for any fall and improved to 0.62 for recurrent falls.

Discussion: Falls remain highly prevalent in communitydwelling older Canadians. Classical retrospective prediction models performed poorly in this setting.

Conclusion: Models might improve significantly with innovative algorithms such as deep neural networks. This would in turn allow targeting specific risk factors to curb fall risk.

\section{The 'Cognitive Vital Sign' (CVS): A Qualitative Study Into the Feasibility of Implementing a New Delirium Screening Tool on the Acute Care of the Elderly Unit in London, Ontario}

R. Kyle, L. Diachun, J. Thain, T. Wong, N. O'Regan. Division of Geriatric Medicine, Department of Medicine, Western University.

Background: Delirium is common in older inpatients and is independently associated with poor outcomes. Screening is recommended; however implementation is fraught with barriers. This study aimed to explore these in a clinical setting. 
Methods: CVS delirium screening protocol was introduced on the Acute Care of the Elderly unit in a tertiary care hospital. Six-months post implementation, staff perceptions of the protocol were explored using semi-structured interviews until data saturation. Theoretical Domains Framework guided data analysis and was used to identify recurring themes.

Results: Analysis of ten interviews revealed routine delirium screening was perceived to have a positive impact on patient care. Facilitators included knowledge (delirium is a serious consequence of underlying pathology); skills (person-centred nursing care, emotional intelligence to read and respond appropriately to individual patient needs); environmental context/resources (ease and design of the screen, visual reminders for cuing) and social/professional role and identity (delirium screening, treatment and teaching became an important role). Barriers were also identified in these domains: knowledge (limited appreciation of varied delirium presentations); skills (differentiating comorbidities from delirium); environmental context/resources (timing of screen; high rates of comorbid dementia); and social/professional role and identity (not being valued as a team member). Emotional response was an additional barrier (patient/carer fear of discovering abnormal mental status).

Discussion: Interestingly, all facilitator domains were also barriers, suggesting these areas would be high yield to focus on when implementing a new screen. The themes also reveal that a thorough understanding of the clinical context of those domains on each ward would aid successful implementation.

Conclusion: The CVS was feasible and generally well accepted by front-line nursing staff. These insights may facilitate routine delirium screening.

\section{FINALISTS FOR THE EDMUND V. COWDRY AWARD}

\section{Markov Decision Analysis of the Cost-Utility for Antithrombotic Therapy in Older Patients with Atrial Fibrillation at Risk for Falls}

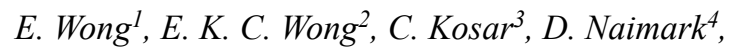
S. E. Straus ${ }^{5}$, H. C. Wijeysundera ${ }^{6} .{ }^{1}$ University of Toronto; ${ }^{2}$ Knowledge Translation Program, Li Ka Shing Knowledge Institute, St. Michael's Hospital; Institute for Health Policy Management and Evaluation, Dalla Lana School of Public Health, University of Toronto; ${ }^{3}$ Institute for Health Policy Management and Evaluation, Dalla Lana School of Public Health, University of Toronto; ${ }^{4}$ Division of Nephrology, Sunnybrook Health Sciences Centre, University of Toronto, Knowledge Translation Program; ${ }^{5}$ Li Ka Shing Knowledge Institute, St. Michael's Hospital; ${ }^{6}$ Division of Cardiology and Cardiac Surgery, Schulich Heart Centre, Sunnybrook Health Sciences Centre, University of Toronto.
Background: Atrial fibrillation (AF) is a common cardiac condition in older adults that results in an increased risk of stroke. Anticoagulants decrease stroke risk associated with AF but increase bleeding risk. Older patients are often at high risk of falls, which further compounds bleeding risk. The purpose of this study was to determine the most cost-effective anticoagulant for older adults with $\mathrm{AF}$ at high risk of falling.

Methods: Using a decision analytic Markov model in a twodimensional simulation, quality-adjusted life years (QALYs), total cost, and incremental cost-effectiveness ratios (ICERs) were calculated for each medication (ASA, warfarin, apixaban, dabigatran, rivaroxaban, and edoxaban) based on a distribution of older patients at risk of falls with atrial fibrillation. The model used the Ontario (Canada) public payer perspective and a lifetime horizon. Outcomes included life years (LY), quality-adjusted life years (QALY), costs and incremental cost-effectiveness ratios (ICERs).

Results: The use of direct oral anticoagulants (DOACs) led to overall longer life expectancy $(6.87-7.45 \mathrm{LY})$ than ASA (6.37 LY) and warfarin (6.65 LY). When utilities were included, DOACs (5.03-5.92 QALYs) remained superior to ASA (4.98 QALYs). The most cost-effective antithrombotic therapy for atrial fibrillation in older patients at risk of falls is apixaban, with an ICER of C\$8,621 per QALY gained (5.92 QALYs at C\$94,304). It is a dominant strategy over warfarin and other DOACs.

Discussion: Strengths of this study include derivation of efficacy parameters from a network meta-analysis of randomized trials and validation of the model against external cohorts. Limitations of this study include dichotomizing stroke and bleeding risk into high/low categories without simulating individual risk factors.

Conclusion: Apixaban is the most cost-effective antithrombotic in older individuals at high risk of falls from a public payer perspective.

\section{Access to Community-Based Specialized Geriatric Care in Ontario: Proposing a Decision-Support Tool to Facilitate the Referral Process}

\section{S. Hogeveen ${ }^{1}$, G. Heckman ${ }^{2}$, K. Milne ${ }^{3}$, J. Hirdes ${ }^{2}$. ${ }^{1}$ McMaster University; ${ }^{2}$ University of Waterloo; ${ }^{3}$ Regional Geriatric Programs of Ontario.}

Background: The role played by geriatric medicine (GM) in caring for seniors in the community with complex needs, such as those receiving publicly-funded home care $(\mathrm{HC})$ services, has not been well described in Ontario. Study objectives were to explore service use patterns, outcomes, and characteristics associated with GM contact, and to propose a mechanism for more equitable referral and access to care.

Methods: This was a population-based retrospective cohort study of older HC clients in Ontario ( $\mathrm{N}=196,444 ; 2012-2015)$. 
Standardized HC assessments were linked to health administrative datasets (out-patient physician and acute care services use). Descriptive statistics were used to describe service use patterns. Multivariable generalized estimating equation modeling was used to examine associations between client characteristics and outpatient GM contact in 90 days postassessment. Based on results, provincial stakeholders created a standardized decision-support tool to identify $\mathrm{HC}$ clients who would most benefit from GM referral.

Results: $49.6 \%$ of older $\mathrm{HC}$ clients had $\geq 4$ physician contacts post-assessment; only 5.2\% had GM contact. In the final multivariable model (region-adjusted), female sex, difficulties accessing home, impaired locomotion, recovery potential, hemiplegia/hemiparesis, and cancer were associated with lower odds of GM contact. Age, worsened decision-making, dementia, hallucinations, Parkinsonism, osteoporosis, and caregiver distress/institutionalization risk were associated with higher odds of contact. A tool was proposed that identifies $\mathrm{HC}$ clients at risk for caregiver distress/institutionalization (based on current practice), with medical instability and complexity (based on provincial expertise), and needs within the Geriatric 5Ms for GM referral.

Discussion: Few older HC clients were in contact with community-based GM, despite having complex medical needs and frequent contact with the health system.

Conclusion: A decision-support tool could inform workforce planning, improve care provider collaboration, and promote more rational and equitable resource allocation.

\section{Study of the Factors Influencing Implementation of Four Frailty-Screening Tools in Family Medicine Clinics in Canada-Provisional Results:}

O. G. Acakpo ${ }^{1}$, S. Maranda-Pelletier ${ }^{1}$, J. Elliott ${ }^{2}$, A. Costa ${ }^{3}$, O. Theou ${ }^{4}$, P. Stolee ${ }^{2}$, G. Heckman ${ }^{2}$, M-J. Sirois ${ }^{1}, K$. Rockwood $^{4}$, P. McPhedran ${ }^{2}$, A. Kernoghan ${ }^{2}$, A. Giguère ${ }^{1}$.

${ }^{1}$ Laval University; ${ }^{2}$ University of Waterloo; ${ }^{3}$ McMaster University; ${ }^{4}$ Dalhousie University.

Background: Early detection of frailty is required to trigger the implementation of individualized care plan. Hence, this project will describe the factors influencing implementation of four frailty-screening tools in family medicine clinics.

Methods: This ongoing mixed-methods study uses focus groups and questionnaires with healthcare providers (HCPs) from four family medicine clinics in Quebec, Ontario and Alberta, and community-based individuals aged 70 years old or more, to describe perceptions about the InterRai Preliminary Screener, the Pictorial Fit-Frail Scale, the Clinical Frailty Scale (CFS), and the PRISMA-7. The qualitative thematic analysis is based on the Theoretical Domains Framework. HCPs rate each tool with the System Usability Scale to compare them using a repeated measure linear regression.
Results: To date, five focus groups were completed in Quebec with a total of 14 physicians, eight nurses, and eight older adults (mean age: $74 \pm 3$ years). Most HCPs were women (91\%), with under five years of practice experience $(27 \%)$. Facilitators to the tools' implementation comprised having clear instructions on the next steps, comprehensiveness, giving a total score, brevity, lay language with pictures, and having time to complete them. CFS was rated as the most usable of the four tools by HCPs $(\mathrm{P}<0.0001)$.

Discussion: Most of the identified factors are related to the clinical setting and suggest practical strategies to implement the tools effectively. The factors identified related to HCPs suggest that training will be mandatory for implementation. Some of the identified factors are related to the tools' characteristics and require adapting the items to fit the studied setting better.

Conclusion: The focus group interviews in Alberta and Ontario are still ongoing. Findings will inform recommendations on implementing frailty screening in family medicine clinics.

\section{Pre-Existing Frailty is not Associated with 60-Day Mortality in Geriatric Medicine Inpatients}

S. Searle, S. D. Searle, H. L. Ellis, E. Palmer, K. Rockwood, D. Davis. University College London.

Background: A fundamental tenet of inpatient geriatric medicine is the early identification of frailty in order to predict recovery and adverse outcomes. Specific, quantitative measures for the recovery of frail patients are not known. Using a subclinical frailty marker, we: (1) Modelled patient recovery and; (2) Using (1) with the Clinical Frailty Scale (CFS) determined whether chronic frailty remains associated with mortality.

Methods: This was a secondary analysis of a prospective cohort study of inpatients under acute geriatric medicine care. Routine investigations were used in a frailty index (FI-Lab), which was measured over the duration of inpatient stay. Correcting for age, sex and CFS, a joint random effects model (for FI-Lab) and cox proportional hazards model (mortality censored at 60-days) was used.

Results: The cohort consisted of 1341 admissions. The mean age was 84.9, and median CFS 6. In the FI-Lab model, frailer individuals had a worse initial FI-Lab score $(\mathrm{p}<0.001)$ and improvements in the FI-Lab were dependent on the CFS $(p=0.014)$. Sixty-day mortality was associated with the FI-Lab $(p<0.001)$, the FI-Lab's slope $(p=0.001)$ and age $(p<0.001)$. The CFS ( $\mathrm{p}=0.09$ ) was not associated with mortality.

Discussion: This shows that in acute illness baseline frailty is associated with inpatient FI-Lab trajectories, but not mortality. These findings conform to the pre-existing theory of how frailty impacts acute illness. It is unclear as why quantitative tools for recovery, like the FI-Lab, are not studied more for validation, the aiding of clinical decision making, 
identification of high-risk patients in a healthcare system with limited geriatric expertise, and the timely communication of patient progress to patients and family members.

Conclusion: In hospital measurement of the FI-Lab supersedes any association between chronic frailty and 60-day mortality.

\section{FINALISTS FOR JUNIOR FACULTY PRESENTATIONS}

\section{Urinary Incontinence Among Indigenous Seniors in Northern Canada \\ W. Gibson, S. Nazari, K. Kerber, F. Kolahdooz, S. Sharma, A. Wagg. University of Alberta.}

Background: Urinary incontinence is common and increases in prevalence with increasing age in both men and women. Although numerous studies have examined the prevalence among the general population in Western countries, the prevalence of incontinence among Indigenous Seniors in Canada's north, a vulnerable and under-served group, is unknown.

Methods: As part of a wider research project on health in Indigenous seniors (50+ years) in Inuvik, Northwest Territories, continence status and associated comorbid conditions were gained via structured interviews. A convenience sample was recruited by word of mouth, representing approximately $80 \%$ of the population. Participants gave informed consent.

Results: The mean age of participants was 65.5 years (SD 8.9). Of the 71 people recruited, 33 (46\%) reported at least weekly incontinence. More women reported incontinence than men $(60 \%$ vs. $29 \%)$. The prevalence of diabetes and myocardial infarction did not differ between participants who were continent and participants with incontinence ( $21 \%$ vs. $18 \%$ and $15 \%$ vs. $16 \%$, respectively); there was a higher prevalence of stroke in participants with incontinence ( $9 \%$ vs. $5 \%$ ).

Discussion: Indigenous seniors have a high prevalence of incontinence among women. This is greater than that reported from previous studies among non-Indigenous Canadians. The underlying reasons for this were not assessed here, but may include obstetric practice and the high rates of diabetes, known risk factors for incontinence. The rate of incontinence in men was low; this may be due to under-reporting. There were no obvious associations with recorded comorbidities.

Conclusion: Incontinence is a common problem for female Indigenous seniors who may have limited access to specialist assessment and treatment.

\section{Frailty in Parkinson's disease: A Scoping Review}

J. M. McMillan ${ }^{1}$, Q. Michalchuk ${ }^{2}$, Z. Goodarzi ${ }^{3}$.

${ }^{1}$ University of Calgary; ${ }^{2}$ University of British Columbia;

${ }^{3}$ University of Calgary, Department of Medicine, Section of Geriatric Medicine.
Background: Frailty and Parkinson's disease (PD) are common conditions that increase with age. Independently, frailty and PD lead to increased morbidity and mortality for patients. Few studies report on frailty in patients with PD and there is a critical lack of evidence for the detection and management of frailty in PD. We performed a scoping review to determine the prevalence, associations and outcomes of frailty in persons living with PD.

Methods: We searched four electronic databases from inception to May 13, 2019 for articles which reported the prevalence, associations and outcomes of frailty in persons living with PD. Review of titles and abstracts, full text citations and data extraction was performed in duplicate.

Results: Nine-hundred and eighty-four citations were identified, of which 99 full-text articles were reviewed. Seventeen citations met inclusion criteria and are summarized here. The prevalence of frailty in PD was approximately one-third ( 27.9 to $35.6 \%$ ) however there were outliers. The most common frailty screening measures were the frailty phenotype, Clinical Frailty Scale, and frailty index. Frailty in PD was associated with recurrent falls, cognitive impairment, dementia, orthostatic hypotension, fatigue, hallucinations, nursing home placement, dependency in activities of daily living and in-patient mortality. PD characteristics, including disease duration, motor impairment, non-tremor dominant PD (postural instability/gait difficulty dominant phenotype) and total dose of levodopa were associated with frailty.

Discussion: There is no agreed upon screening tool for identifying frailty in PD, however, the importance of its identification is apparent given the association between frailty and adverse outcomes in persons with PD.

Conclusion: Future studies are required to guide clinicians caring for persons with PD in how best to identify and tailor the management of their frail patients living with PD.

\section{Exploration of Residents' Perception and Responses to the Multiple Dimensions of Clinical Complexity when Caring for Older Adults}

\section{J. Thain, L. Diachun, L. Lingard, S. Cristancho. Western} University.

Background: Managing complex situations is a core feature of physician competency. However, what constitutes 'complexity in clinical medical practice' is not well defined. With implementation of CBME, establishing key features for competency is required. Previous studies involving Attendings and Medical Students demonstrated that complexity extends beyond the medical dimension. Resident perceptions have yet to be explored.

Methods: Constructivist grounded theory was used to inform data collection and analysis. 22 residents PGY-2+ were recruited. Residents were asked to draw Rich pictures, a graphic elicitation technique, based on complex older adults they have cared for. 
This was used as the basis for discussion and a semi-structured interview. Recorded interview transcripts were analyzed iteratively, identifying themes until data sufficiency was reached.

Results: Two dominant types of complexity emerged medical and social. Their level of challenge over time was paradoxical. Medical complexity was initially perceived as challenging but became progressively easier. Conversely, social complexity became increasingly challenging. Factors associated with this increased challenge included paucity of training in medical school with little exposure during early residency, followed by an abrupt transition in senior residency, where residents are expected to competently navigate socially complex issues. This is compounded by a discharge focused hidden curriculum pervasive within hospital culture.

Discussion: Residents are caught in a dichotomy of values between the taught curricula and hospital priorities. Medical education values medical complexity with little emphasis on social complexity. With constrained resources, hospitals highly value patient flow. Residents, with poor foundational knowledge, are expected to manage socially complex issues to facilitate timely discharges, leading to feelings of frustration, overwhelm and failure.

Conclusion: Social complexity should be considered when designing curricula and key features for determining competence for 'complexity in clinical medical practice'.

\section{Distinct Clinical and Sociodemographic Features of Persons Newly Diagnosed with Human Immunodeficiency Virus (HIV) Younger Versus Older Than 50 Years of Age at Diagnosis}

J. M. McMillan ${ }^{1}$, L. H. Rubin ${ }^{2}$, M. J. Gill'. ${ }^{1}$ University of Calgary; ${ }^{2}$ Johns Hopkins University School of Medicine;

${ }^{3}$ University of Calgary, Department of Medicine.

Background: In Canada in 2017 17\% of new diagnoses of Human Immunodeficiency Virus were in persons $>50$ years. At the Southern Alberta Clinic, the proportion of persons newly diagnosed with HIV over 50 years of age has increased from $7 \%$ in 1990 to $18 \%$ in 2019 .

Methods: We compared clinical and sociodemographic factors between patients newly diagnosed with HIV between the ages of 18 and 50 years and those $>50$ years of age in Southern Alberta.

Results: Older adults $>50$ are more likely to present with lower CD4 T-cell counts than adults diagnosed between 18-50 years of age. The indication for performing the HIV test differed between the two age groups with older adults more likely to be tested due to illness (47\%) and younger adults more likely to request the test $(34 \%)$. The reported risk for HIV acquisition also differed with heterosexual sex the largest risk category for older adults (29\%) and men who have sex with men the largest risk category for younger adults (51\%). Differences were also observed in relationship status and living arrangements between the two age groups. One-third of older adults newly diagnosed with HIV were married to an opposite sex partner in contrast to $12 \%$ of $18-50$-year-olds. Older adults were also more likely to live alone than those diagnosed between 18-50-years. No differences were observed between the age groups by race/ethnicity or sex.

Discussion: In Southern Alberta 18\% of new HIV diagnoses in 2019 were in persons $>50$ years of age. There are important differences between patients diagnosed with HIV over 50 years of age compared to those diagnosed between 18 and 50 years of age.

Conclusion: Health care providers should consider HIV testing in older patients to avoid a delayed diagnosis as well as recognize the unique clinical and sociodemographic differences from those diagnosed at a younger age.

Comparative Efficacy of Interventions for Reducing Depressive Symptoms in Dementia: A Systematic Review and Network Meta-Analysis

J. Watt ${ }^{l}$, Z. Goodarzi ${ }^{2}$, A. A. Veroniki ${ }^{3}$, A. Tricco ${ }^{l}$, S. Straus ${ }^{1} .{ }^{1}$ University of Toronto; ${ }^{2}$ University of Calgary; ${ }^{3}$ University of Ioannina.

Background: Depressive symptoms are common in persons with dementia; the comparative efficacy of pharmacologic and nonpharmacologic interventions for reducing depressive symptoms in dementia is unknown.

Methods: We searched MEDLINE, Embase, CENTRAL, CINAHL, PsychINFO, and grey literature between inception and December 4, 2019 for randomized trials of pharmacologic or nonpharmacologic interventions targeting depressive symptoms in persons with dementia. Pairs of reviewers, working independently, conducted study screening, data abstraction, and risk of bias appraisal. We conducted Bayesian random-effects network meta-analyses (NMAs) and pairwise meta-analyses to derive standardized mean differences and back-transformed mean differences (MD; on the Cornell Scale for Depression in Dementia [CSDD]).

Results: After screening 20285 titles and abstracts, we included 232 studies (27140 patients) in our systematic review. The minimum clinically important difference on the CSDD was 2.05 at 0.4 standard deviations. In our primary NMA (193 studies, 57 interventions, 18482 patients), cognitive stimulation (MD -2.97, $95 \%$ credible interval [CrI] -4.6 to -1.35 ), cognitive stimulation + cholinesterase inhibitor (MD $-11.06,95 \% \mathrm{CrI}-18.12$ to -3.97), massage therapy (MD $-8.34,95 \% \mathrm{CrI}-12.53$ to -4.21 ), occupational therapy (MD $-2.57,95 \% \mathrm{CrI}-4.89$ to -0.33 ), and reminiscence therapy (MD $-2.11,95 \% \mathrm{CrI}-3.73$ to -0.55 ) were more efficacious than usual care for reducing depressive symptoms. In pairwise meta-analyses, exercise (MD -2.27, 95\% CrI -4.32 to -0.22 ) and multidisciplinary care (MD - 2.42, $95 \%$ CrI -4.68 to -0.15 ) were also more efficacious than usual care.

Discussion: Our results show that nonpharmacologic and multidisciplinary care approaches are efficacious for reducing 
depressive symptoms in persons with dementia; whereas, pharmacologic approaches alone were not more efficacious than usual care.

Conclusion: Clinicians should prioritize nonpharmacologic and multidisciplinary care approaches for reducing depressive symptoms in persons with dementia.

Improving Equitable Access to Specialist Advice: A Case Study of an eConsult Service in Long Term Care

C. Fung ${ }^{1}$, C. Levi' ${ }^{2}$, S. Shah ${ }^{3}$, M. Helmer-Smith ${ }^{4}$, C. Liddy ${ }^{5}$

${ }^{1}$ Dept of Family Medicine, University of Ottawa; ${ }^{2}$ The

Ottawa Hospital; ${ }^{3}$ University of Ottawa; ${ }^{4}$ C.T. Lamont

Primary Health Care Research Centre, Bruyère Research

Institute; ${ }^{5}$ C.T. Lamont Primary Health Care Research

Centre, Bruyère Research Institute; Dept of Family

Medicine, University of Ottawa.

Background: Residents in long term care (LTC) face unique challenges accessing specialist advice, including barriers to attending outside appointments; limited access to diagnostic tools; and poor care coordination. They may only receive specialist care when urgent or emergent situations arise. Electronic consultation (eConsult) is a secure, asynchronous, web-based tool that allows primary care providers (PCPs) timely access to specialists' advice on behalf of residents. In community practice, eConsult has demonstrated reduced wait times for advice, improved quality of care, avoided faceto-face referrals, and lower costs. Little is known about the clinical utility of eConsult in LTC.

Methods: This is a descriptive case based study. All eConsult requests submitted by primary care providers on behalf of LTC residents from Jan. 2017 through to Dec. 31, 2018 are being categorized using a modification of the International Classification for Primary Care (ICPC-2) taxonomy. Question type (e.g., diagnosis or management) was classified using a validated taxonomy.

Results: 113 cases were submitted by LTC PCPs to 23 specialty groups through the Champlain BASE ${ }^{\mathrm{TM}}$ eConsult Service between January 1, 2017 and December 31, 2018. Initial review of the case content identified most common specialty areas accessed, question type and type of advice given. The provision of actionable advice appropriate to the care setting, caregiver reassurance, and avoided transfer were observed. All cases were answered within 1 week.

Discussion: eConsult permits LTC PCPs to obtain timely, specific and appropriate recommendations in response to their clinical questions. Access to this advice limits the stress associated with external transfer for residents, caregivers, and PCPs.

Conclusion: eConsult supports clinical decision-making and improves access to specialist advice for the most vulnerable seniors living in long term care.

\section{POSTERS}

\section{Reliability of Activity Trackers for Monitoring Sleep in Hospitalized Patients with Neurocognitive Disorders}

M. Ahuja ${ }^{1}$, S. Siddhpuria ${ }^{2}$, J. Gormley ${ }^{l}$, C. Reppas ${ }^{3}$, J. Lee ${ }^{1}$, E. Wong ${ }^{3}$, C. Patterson ${ }^{1} .{ }^{1}$ McMaster University;

${ }^{2}$ University of British Columbia; ${ }^{3}$ University of Toronto.

Background: The reliability of wrist sleep trackers in patients with cognitive disorders is unclear. We investigated the consistency of activity monitor data in patients with a diagnosis of mild cognitive impairment (MCI), dementia, Parkinson's disease and those who developed delirium.

Methods: This was part of a study investigating sleep in older adults during post-operative recovery from a hip fracture. Wrist activity monitors $\left(\mathrm{Fitbit}^{\mathbb{R}}\right.$, Alta 2 ) were worn for the duration of their hospital stay. We conducted an exploratory analysis of the device data from participants with MCI, dementia, Parkinson's disease and those who developed postoperative delirium. The proportion of sleep data that failed to be recorded from participants with these conditions was compared to data from other participants in the study.

Results: 67 (66\% female) patients were enrolled in the study with an average age of 83 years old. Data analysis demonstrated that activity monitors did not record data in $48 \%$ of nights (73 of 151) in the group with cognitive disorders (22 patients) compared to just $22 \%$ (67 of 310 ) in those without a cognitive diagnosis (45 patients).

Discussion: Sleep data tracking using wrist monitors in our study was inconsistent in participants with cognitive disorders. Possible reasons for the discrepancy include agitation, tremors, excessive movements (e.g. hyperactive delirium) or device removal. The reliability of these devices for research or lifestyle use in populations with dementia and other cognitive disorders is potentially questionable.

Conclusion: Our results highlight the potential challenges of using devices validated for healthy adults and applying them to older adults with physical or cognitive impairments. Alternative methods may be required to reliably and accurately evaluate sleep in future research investigations involving this patient population.

The Clinical State and Correlates of Sero-Positive Elderly Nigerians: A 10 Year Retrospective Review of Care in Federal Teaching Hospital, Ido Ekiti, Ekiti State, Nigeria

E. K. Ariyibi, O. E. Omosanya, O. T. Elegbede, O. Ajetunmobi, A. T. Omolayo, A. M. Fashola, L. A. Adebusoye, E. A. Ajayi. Federal Teaching Hospital, Ido-Ekiti, Ekitit State, Nigeria. 
Background: The study aimed at determining the proportion of people living with HIV/AIDS who attended HIV clinic in the last 10 years who were elderly patients (aged 60 years and above), and to provide an overview of their clinical status - as regards their BMI, CD4+ count, and viral load. It also aimed at elucidating the patterns of co-morbidity, duration of being on HAART and the present state/last clinic attendance of sero-positive elderly people

Methods: The study lasted for a month and a half. It was carried out at the HIV clinic of the Federal Teaching Hospital Ido-Ekiti, Ekiti State Nigeria, using the case files of seropositive elderly patients who were 60 years or attained the age of 60 years during the period under review as the study population. A retrospective review of data in the case files of the study population was done during the study period. The data reviewed were analyzed using SPSS version 17.

Results: Elderly persons constituted about 9.58\% (135) of the total number of patients (1409) seen over the period. About $49.62 \%$ (67) and 50.38\% (68) were elderly women and men respectively. BMI dropped in over $60 \%$ (81) of the clients seen, while the CD4+ reduced with about $10 \%$, the viral load increased substantially with nearly $25 \%$ after using HAART for an average of eight years. Duration of being on HAART ranged from seven to nine years for about $60 \%(81)$ of patients while a little over $29.6 \%$ (40) were most likely lost to follow-up or died during the period of receiving care. Side-effects reported by the elderly sero-positive accounted for about $6.66 \%(9)$, while comorbidity of a minimum of one to a maximum of three non-communicable disease(s) were seen in about $65.18 \%$ (88) of the elderly sero-positive seen.

Discussion: Elderly people should be part of the vulnerable groups in HIV/AIDS care because the overall quality of life of sero-positive elderly individuals can be improved upon by HAART.

Conclusion: Additional supportive care should be strongly advocated in view of the decreased BMI and presence of comorbidity noticed in elderly patients.

\section{The Role of Family Oriented Interview and Counselling on Activities of Daily Living Amongst Elderly Patients Attending Family Medicine Clinic of Federal Teaching Hospital Ido - Ekiti.}

E. K. Ariyibi ${ }^{1}$, O. T. Elegbede ${ }^{1}$, O. E. Omosanya ${ }^{1}$,
O. M. Shabi ${ }^{1}$ L. A. Adebusoye ${ }^{2}$, E. A. Ajayi.${ }^{I}$ Federal
Teaching Hospital, Ido-Ekiti, Ekitit State, Nigeria;
${ }^{2}$ Geriatric Centre, University College Hospital, Ibadan,
Oyo State, Nigeria.

Background: Elderly persons are increasing in numbers globally and their functional ability is depreciating un-abated as their age increases. The study aimed at determining the role of family oriented interview and counselling (FOIC) on ADL in elderly respondents, and factors affecting ADL in the elderly.
Methods: A randomized, controlled interventional study was carried out on one hundred (100) elderly patients attending Family Medicine Clinic of the Federal Teaching Hospital, Ido - Ekiti, Nigeria. Simple random sampling was used to select respondents who were grouped into control and intervention groups. Their Basic ADL (BADL) and Instrumental ADL (IADL) were assessed by use of Katz and Lawton instruments, respectively, before and after intervention. Interventions given varied according to respondent's assessment/needs, and were agreed upon and supported by their family members. The study lasted for two months. Data collected were analysed by SPSS version 17 .

Results: The mean of BADL increased from $4.0 \pm 1.9$ to 5.6 \pm 1.0 along with a similar increase in the mean of IADL from $5.6 \pm 2.1$ to $7.1 \pm 1.6(\mathrm{p})$ in the intervention group and were statistically significant, while the increase in the mean of BADL ( $4.6 \pm 1.9$ to $4.9 \pm 1.6)$ and IADL $(6.1 \pm 2.1$ to $6.3 \pm 2.0)$ in the control group were insignificant $(\mathrm{p}<0.05)$.

Discussion: Elderly persons' families are very important in helping to optimize activities of daily living (ADL) - self-care and independent living in elderly persons. The study further enhances the roles of family in the healthcare of her members especially the elderly ones.

Conclusion: The study demonstrated that FOIC had a significant effect on ADL amongst the elderly. Diseases (noncommunicable and infectious diseases) and old age amongst others were factors that affected ADL in the elderly.

\section{A Community-Based Workshop to Address Dementia-Related Stigma: Preliminary Findings from a Rural Community}

\author{
J. Bacsul , M. Viger ${ }^{2}$, S. Johnson ${ }^{3} .{ }^{1}$ University of Regina; \\ ${ }^{2}$ University of Saskatchewan; ${ }^{3}$ University of Alberta.
}

Background: Recently, the Government of Canada released a national dementia strategy that addressed the need reduce stigma to improve the quality of life for people with dementia. Dementia-related stigma can delay early dementia diagnosis and lead to low self-esteem, social isolation, depression, and suicide. This presentation aims to examine the development and evaluation of a community-based presentation to support education and awareness of dementia-related stigma in rural communities.

Methods: Developed in collaboration with rural older adults and community leaders, the presentation included a dementia definition, different dementia types, warning signs and symptoms, risk reduction strategies, information on dementia-related stigma and myths, and community-based interventions/strategies to reduce dementia-related stigma.

Results: Many of the older adults attending the presentation had personal experiences as family members, friends, care partners or as a person affected by dementia or other forms of cognitive impairment. The majority of older adults attending 
the workshop felt that it improved their knowledge, attitude, comfort level, and ability to recognize the signs and offer support to someone living with dementia. Almost all of the participants reported that they would recommend the presentation to other rural communities.

Discussion: This community-based presentation was found to be beneficial for improving knowledge, attitudes and awareness of dementia in a rural community. Additional research is needed to develop, implement, and evaluate interventions for improving dementia knowledge and awareness for different cultures and contexts (e.g., rural, remote, urban).

Conclusion: There is a growing need for more dementia education and awareness strategies, especially in rural and remote communities. Community-based collaboration and partnerships with older adults affected by dementia is essential to developing interventions to address dementia-related stigma and improve quality of life for people with dementia and their care partners.

\section{Addressing Dementia-Related Stigma in Rural Communities: From Challenges to Solutions}

\section{J. Bacsu ${ }^{1}$, S. Johnson ${ }^{2}$, M. Viger ${ }^{3} .{ }^{1}$ Faculty of Kinesiology and Health Studies, University of Regina; ${ }^{2}$ University of Alberta; ${ }^{3}$ University of Saskatchewan.}

Background: Age is the greatest risk factor for dementia, and the number of rural older adults is increasing in Canada. Although dementia-related stigma is a widely documented issue, few studies focus on ways to address stigma, especially within rural communities. This presentation aims to: explore the contributing factors of dementia-related stigma in rural communities; and identify interventions to reduce stigma of dementia in rural communities.

Methods: Drawing on a community-based research approach, data were collected through semi-structured interviews with 18 older adults, and a focus group with 7 community leaders (e.g., representatives from local businesses, seniors' centre and other community-based organizations) in rural Saskatchewan, Canada. Guided by solutions-focused theory, data were coded using thematic analysis.

Results: Contributing factors of dementia-related stigma ranged from fear to lack of dementia knowledge. Several strategies were identified to address dementia-related stigma: forming support groups; hosting educational workshops; inviting guest speakers with dementia; talking openly about dementia; learning more about dementia; asking questions; being a leader and sharing your lived-experiences; being inclusive; developing intergenerational programs; and avoiding assumptions and hurtful jokes.

Discussion: Rural older adults identified a wide range of interventions and strategies to reduce stigma of dementia. Further research is necessary to explore the feasibility, implementation and evaluation of different anti-stigma strategies within rural communities.

Conclusion: As Canada's rural population ages, there is a growing need for community-based interventions to address stigma of dementia. Engaging in rural partnerships and collaborative research is essential to developing culturally and communityinformed strategies to reduce dementia-related stigma and improve quality of life for people affected by dementia.

\section{Moving Professionalism into the Formal Geriatrics Curriculum}

\section{A. Berger. University of Toronto, University Health Network and Sinai Health System.}

Background: Professionalism: you know it when you see it, but how do you teach it? A systematic review of the literature on professionalism education in postgraduate medical education showed that many types of teaching modalities, most often small-group discussions, could indeed improve resident professionalism.

Methods: Drawing from the literature and local expertise, we began implementing a series of academic half days (AHD) for geriatric medicine residents (PGY4-5) at the University of Toronto. We discussed topic ideas with resident representatives to prioritize topics and ensure relevance to their experience. The curriculum is guided by the CanMEDS competency framework and includes the following sessions:

- Professionalism, Commitment to Patients: Person-Centered Care and Skills for Shared Decision-Making

- Professionalism, Commitment to Self: Professional Identity and Physician as Self

- Professionalism, Commitment to Society: The Social Contract and Medico-legal Aspects of Medicine

- Communication: Advanced communication skills workshop for navigating complex discussions (driving cessation, family-health care team conflict)

- Leadership: Knowing Yourself to Lead Others

- Collaboration: Managing Difficult Relationships

- Advocacy: Stronger Together- Allyship in Medicine

Results: We have implemented four of the seven planned sessions so far. Learners rated these AHD sessions as "excellent" and "outstanding" on formal teaching evaluations, and they scored an average of 4.8/5.

Discussion: Our results indicate that learners are eager for opportunities to reflect upon challenges in practice and gain skills to excel as medical leaders.

Conclusion: Including professionalism and other non-medical expert competencies into the formal curriculum is highly rated by trainees. Future work will focus on formal program evaluation based in transformative learning theory to better understand what trainees gained from this experience. 
Geriatric Specialist Physician Human Resources in Ontario: 2018 and Projected to 2025

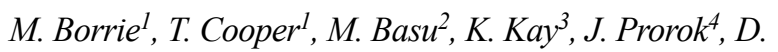
Seitz ${ }^{5}{ }^{1}$ St. Joseph's Healthcare London; ${ }^{2}$ University of Toronto; ${ }^{3}$ Provincial Geriatrics Leadership Office (PGLO); ${ }^{4}$ Department of Psychiatry, Queen's University; Providence Care, Mental Health Services; ${ }^{5}$ University of Calgary/Queen's University; Providence Care, Mental Health Services.

Background: The number of older adults with complex health needs in Ontario is growing. We conducted a physician human resource mapping project to assess the existing 2018 and projected 2025 number of geriatric specialist physician resources within Specialized Geriatric Services (SGS) and Seniors Mental Health (SMH) across Ontario.

Methods: Geriatric specialist physicians in this study were geriatricians, geriatric psychiatrists, and Care of the Elderly (COE) physicians. We determined the existing number of geriatricians, geriatric psychiatrists, and $\mathrm{COE}$ physicians working within each of the Local Health Integration Network (LHINs). We confirmed the clinical full-time-equivalent status (CFTE) for geriatric medicine and psychiatry specialists to assess accurate clinical resources. We projected the number of new trainees anticipated to enter practice and the number of physicians estimated to retire between 2018-25. We compared these numbers and projections against established ratios of 1.175 geriatricians $/ 10,000$ (population over age 65) and 1 geriatric psychiatrist/10,000 (population over age 65) to assess existing and future need.

Results: Using the ratios, there was a shortfall for the existing CFTE geriatricians (-150.5) and CFTE geriatric psychiatrists (-116.3) In 2025, the projected deficit of CFTE geriatricians will increase to at least $(-210.4)$ and geriatric psychiatrists to (-193.6). This assumes all of the new trainees are CFTE which is unlikely. There was a total of $143 \mathrm{COE}$ physicians in Ontario. Of the total respondents, 30.05 CFTE were in a direct support role within SGS/SMH, 16.4 CFTE in an indirect role and 58.15 CFTE in regular family practice.

Discussion/Conclusion: Below.

Conclusion: There was a significant existing (2018) and anticipated increased future (2025) shortfall in the required number of geriatricians, geriatric psychiatrists and COE physicians to meet the predictable increase in need.

\section{Delirium Case Finding in the Comprehensive Assessment of Neurodegeneration and Dementia Canadian Study (COMPASS-ND)}

M. Borrie ${ }^{1}$, S. Best ${ }^{2}$, N. Elhayek ${ }^{3}$, M. Dasgupta ${ }^{4}$, K. Rockwood ${ }^{5}$, D. Davis ${ }^{6}$, N. ORegan ${ }^{4}{ }^{1}$ Parkwood Institute, Lawson Health Research Institute; ${ }^{2}$ Parkwood Research Institute; ${ }^{3}$ Parkwood Institute; ${ }^{4}$ Western University; ${ }^{5}$ Dalhousie University; ${ }^{6}$ University College London.
Background: Delirium is a strong risk factor for incident dementia and worsening cognition. COMPASS-ND is recruiting 2310 participants including 600 normal controls, and 1600 people with various types of neurodegeneration into longitudinal cohorts. Comprehensive measures of clinical characteristics, neurological examination, neuropsychology testing, blood and genetic biomarkers, and MRI brain imaging are collected. A delirium questionnaire based on DSM-5 criteria has been embedded in the COMPASS-ND protocol to screen for delirium through structured telephone interviews with participants and caregivers and validated chart review techniques. This will: (1) estimate the period prevalence of delirium per neurocognitive subgroup; (2) determine how dementia biomarkers predict delirium occurrence; (3) quantify delirium as a predictor of disease progression.

Methods: The delirium questionnaire is administered at the COMPASS-ND baseline and follow-up. More intensive delirium case-finding will be piloted at Parkwood Institute in London, ON through a two-stage approach: (1) more frequent telephone interviews (with participants and caregivers) to both administer the questionnaire and enquire about hospitalizations; (2) case finding by chart review for participants hospitalized. Participants with delirium symptomology will have an expert geriatrician assessment to diagnose probable or possible delirium.

Results: At baseline, $14.9 \%$ of participants had a history of delirium. In addition, a history of delirium was more prevalent in participants with dementia. At follow up, $9.9 \%$ of participants with no prior history of delirium had new incidence of delirium. $24.2 \%$ of those with a prior history of delirium had a repeat incident.

Discussion: Delirium case finding in COMPASS-ND will help clarify the relationship between cognitive impairment and delirium to determine the effect that delirium has on the occurrence and rate of cognitive decline.

Conclusion: Continue to follow the cohort and monitor the relationship between cognitive impairment and delirium.

\section{Ending PJ Paralysis: A Quality Improvement Initiative}

F. Carr ${ }^{1}$, A. Morgan 2 , S. Devapura ${ }^{2}$, C. Williams², J. Symon', C. Howe-Riddell' ${ }^{2}$,A. Miller ${ }^{2}, J$. Boone ${ }^{2}$, K. Ebbage ${ }^{2}$, S. Jadaviji ${ }^{2}$, P. Mathura ${ }^{2} .{ }^{1}$ University of Alberta; ${ }^{2}$ Alberta Health Services.

Background: PJ paralysis refers to the negative effects seen amongst hospitalized patients who remain inactive and within their pajamas in hospital. Despite the many complications associated with $\mathrm{PJ}$ paralysis, it is a common problem within acute care facilities, which needs addressing.

Methods: A 3 month quality improvement study was conducted at the University of Alberta Hospital. The aim was 
for $50 \%$ of patients on ward $5 \mathrm{G} 2$ to get dressed in their own clothing by midday, sat up for all meals, and mobilizing to activities. Our intervention involved providing education and assistance to get patients dressed in their own clothing by midday, up for meals and mobilizing to activities. Measures included: daily percentage of patients dressed in their own clothing and up for all meals, weekly mobilization rates, number of nursing staff required, nursing assessment time, and complication rates (e.g. falls).

Results: 70 patients were included in the study. $14.3 \%$ of patients were dressed in their own clothing daily, and $6.4 \%$ of patients were up for all three meals. The average number of patients mobilized weekly was 4.7. A trend was observed towards decreased falls, with little change observed in staff numbers, nursing assessment time or other complication rates. Patient feedback revealed improvement in their self-identity.

Discussion: While our aim was not met, our intervention revealed improvement in patients' self-identity, consistent mobilization rates, a trend towards decreased falls, with little change observed in complications, number of staff or care assessment duration.

Conclusion: Our simple intervention had a positive impact in several areas, with few complications. Post study, this intervention continued, providing evidence of its sustainability and ease of implementation by frontline staff. Future plans include repeating a second cycle next year to determine sustainability and impact.

\section{Decision-Making Capacity Assessment Education}

L. Charles ${ }^{1}$, J. Parmar ${ }^{1}$, S. Brémault-Phillips ${ }^{2}$, T. Kilkenny ${ }^{3}$, M. Johnson ${ }^{3}$, P. G. Tian', O. Babenko ${ }^{4}$, B. Dobbs ${ }^{1} .{ }^{1}$ Division of Care of the Elderly, Department of Family Medicine, University of Alberta; ${ }^{2}$ Department of Occupational Therapy, University of Alberta; ${ }^{3}$ Covenant Health; ${ }^{4}$ Department of Family Medicine, University of Alberta.

Background: With an increasing elderly population, the number of persons with dementia and the number of persons needing decision-making capacity assessments (DMCA) will increase. However, many health care professionals do not feel ready to provide DMCA. Since 2006, we have provided DMCA workshops to allied healthcare professionals and, since 2013, to physicians. We will evaluate the effectiveness of a 3-hour and a 2-day DMCA workshop on self-reported competencies.

Methods: We used a pretest posttest design to evaluate preand post-workshop ratings on 15 competencies using a 4-point scale. Workshop participants consisted of 822 allied healthcare professionals and 281 physicians.

Results: Ten 3-hour workshops were held in 2014-5, with 166 participants. Seven 2-day workshops were held in 2014-8, with 115 participants. There have been 822 recorded allied HCPs participants.
Self-reported Competencies. The physicians' post-workshop ratings were higher (Sign test; $p<0.001$ ) than pre-workshop ratings for all competencies. Five competencies had improved ratings from at least $80 \%$ of participants post-workshop: awareness of problem solving techniques, understanding of the concept of trigger, awareness of legislative acts, confidence in knowledge and skills, and awareness of a system of organization and documentation. Similar results were seen among allied healthcare professionals: an increase in ratings post-workshop. Also, participants agreed that they followed the Model's guiding principles $(90 \%)$, used problem-solving $(92 \%)$, understood discipline-specific roles $(87 \%)$, and were confident in their knowledge of DMCAs (75\%) and pertinent legislation (72\%).

Discussion: The workshops provided skills and information relevant to capacity assessment. The pattern of findings was used to modify the workshops.

Conclusion: There is a need for DMCA training for physicians and allied healthcare professionals. The content and method of the workshops are effective.

\section{Potentially Inappropriate Medication Use in the Elderly}

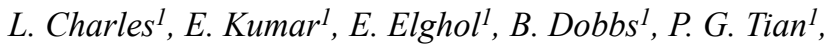
O. Babenko ${ }^{2} .{ }^{1}$ Division of Care of the Elderly, Department of Family Medicine, University of Alberta; ${ }^{2}$ Department of Family Medicine, University of Alberta.

Background: The use of Potentially Inappropriate Medications (PIM) in the elderly population is significant. In 2016, approximately half of Canadian seniors had at least one claim for a drug listed in the Beers Criteria for PIMs. We will determine the prevalence of PIM use among patients referred for comprehensive geriatric assessment (CGA).

Methods: This study consisted of two cross-sectional retrospective chart reviews of patients aged 65 years and older seen at the Outpatient Geriatric Clinics in the Glenrose Rehabilitation Hospital (Glenrose) and Misericordia Community Hospital (Misericordia), Edmonton, Alberta. In 2014, at the Glenrose, we reviewed 200 randomly selected charts from patients seen in 2012-2013; in 2019, at the Misericordia, we reviewed 164 randomly selected charts from patients seen in 2016-2017.

Results: At the Glenrose, the mean age of patients was 79.4 years ( $\mathrm{SD}=7.7$ years). The mean number of medications per patient was $9.0(\mathrm{SD}=4.3)$. The prevalence of PIM use was $45 \%(n=90)$. Of the 90 patients who had PIMs, $46.7 \%(42 / 90)$ had at least one of their medications addressed as a result of the CGA. At the Misericordia, the mean age of patients was 81.8 years ( $\mathrm{SD}=6.8$ years). The mean number of medications per patient was $8.5(\mathrm{SD}=3.9)$. The prevalence of PIM use was $57.3 \%$ (94/164). Of the 94 patients who had PIMs, $47.9 \%$ $(45 / 94)$ had at least one of their medications addressed as a result of the CGA. 
Discussion: Consistent with other studies, we found a high prevalence of PIM use in older adults and a predominance of central nervous system drugs and benzodiazepines.

Conclusion: PIM use in the elderly is prevalent. There is a need for clinical and educational interventions to decrease PIMs use in this patient population.

\section{Changes in Brain Glutathione in Patients with Mild Vascular Cognitive Impairment}

J. J. Chen, J. Song, N. Herrmann, S. E. Black, F. Gao, J. Ramirez, A. C. Andreazza, A. Kiss, P. I. Oh, D. Gallagher, S. Graham, P-S. Wei, K. L. Lanctôt. University of Toronto.

Background: Oxidative stress (OS) is implicated in neurodegeneration and dementia, but little is known about its contributions to prodromal states such as mild vascular cognitive impairment (mVCI). Previously, we reported increased OS in patients with $\mathrm{mVCI}$; however, changes in antioxidant defenses during early cognitive decline are unclear. We will assess brain glutathione (GSH), the primary brain antioxidant, in mVCI participants vs. controls. The relationship of these changes to $\mathrm{S} 100 \mathrm{~B}$, an astrocytic blood marker, will also be assessed.

Methods: Thirty mVCI patients (age 55-85) and 30 age- and sex-matched cognitively-healthy controls will be recruited at entry into a 6-month cardiac rehabilitation program. At baseline, magnetic resonance imaging (MRI) and $1 \mathrm{H}$ magnetic resonance spectroscopy (MRS) will be done to quantify structural changes and brain GSH, which will be assessed using the MescherGarwood Point Resolved Spectroscopy (MEGA-PRESS) pulse sequence in the anterior cingulate cortex (ACC) and the occipital cortex (OCC). Blood biomarkers (S100B and OS markers) will be assessed at baseline. Imaging and blood will also be collected at 6 month follow-up in mVCI participants.

Results: To date, 12 patients ( $\mathrm{mVCI} n=6$, control $\mathrm{n}=6$ ) have been enrolled. Controls vs. mVCI are well-matched: mean age \pm SD $65 \pm 7$ vs. $66 \pm 6$; $\%$ male $83 \%$ vs. $83 \%$; mean baseline Montreal Cognitive Assessment \pm SD $28 \pm 0$ vs. $22 \pm 1$. At baseline, mean $\pm \mathrm{SD}$ GSH for $\mathrm{mVCI}$ were: $\mathrm{ACC}=$ $2.26 \pm 0.31 \mathrm{IU}$ and $\mathrm{OCC}=1.98 \pm 0.11 \mathrm{IU}$; for controls: $\mathrm{ACC}=$ $1.73 \pm 0.30 \mathrm{IU}$ and $\mathrm{OCC}=1.39 \pm 0.04 \mathrm{IU}$.

Discussion: MRS measurement of brain GSH is feasible, more participants are needed to compare group differences.

Conclusion: Study findings will clarify the central antioxidant status in mild VCI and may provide insight into disease mechanism and potential therapeutic targets.

\section{User's Perceptions of an In-home Medication Dispensing System: A Qualitative Study}

\section{Chiu, A. Ahmad, M. Arain. Alberta Health Services.}

Background: In-home medication dispensing technologies could help managing complex medication regimens for older adults. The purpose of the study was to determine users' perspective on a medication dispensing system (MDS) in supporting medication adherence of individuals living at home with chronic conditions.

Methods: This study is a part of a randomized controlled trial on a MDS in a Western Canadian province. We interviewed participants who were recruited into the intervention group and started using a MDS. Maximum variation purposive sampling was used to select interview participants based on age, number of medications, and health conditions.

Results: Thirteen participants were interviewed with an average age of $63.7(\mathrm{SD}=8.2)$ years and taking an average of 8.9 $(\mathrm{SD}=3.6)$ prescribed medications. Most participants were females $(\mathrm{n}=11)$ and all participants were diagnosed with two or more chronic conditions. Four themes emerged: 1) Audio and visual reminders and organized medication supported medication adherence and independence in daily routines; 2 ) Participants found the MDS to be acceptable, although privacy and security were an issue for some; 3 ) The perceived necessity of the MDS was split among participants, with cost being one of the main concerns; and 4) areas of technology improvement included the hard-to-open plastic medication packets and the sometimes inexact recording of medication adherence by the MDS if medications were dispensed on behalf of the patients.

Discussion: Patient support in medication management promotes medication adherence and independence. Technology usability is crucial for the acceptance and adoption of older adults. Related assistive technology would be best fitted for patients physically or cognitively challenged in adhering to medications.

Conclusion: The MDS is an acceptable tool for improving medication adherence in older adults. Increased medication adherence may lead to patient and system-level benefits.

\section{Advance Care Planning in Canadian Nursing Homes: When, Who, What, How?}

N. Choi ${ }^{1}$, H. Keller ${ }^{2}$, V. Vucea ${ }^{2}$, S. King ${ }^{3}$, I. Bains', G. Heckman ${ }^{2}$, P. Quail5, V. Boscart ${ }^{6}$, C. Ramsey ${ }^{\text {I }}$, M. Heyer ${ }^{6}$, A. Garland ${ }^{1}{ }^{1}$ University of Manitoba; ${ }^{2}$ Schlegel-University of Waterloo Research Institute for Aging; ${ }^{3}$ University of Calgary; ${ }^{4}$ University of Waterloo; ${ }^{5}$ Alberta Health Services, University of Calgary; ${ }^{6}$ Conestoga College.

Background: Most Canadian nursing home $(\mathrm{NH})$ residents are frail, with multiple comorbidities and impairments. Thirty to fifty percent of residents die each year and they experience a progressive burden of severe symptoms leading up to their death. Hence, there remain concerns about the adequacy of Advance Care Planning (ACP) and quality of end-of-life (EOL) care in NHs. We sought to identify existing ACP practices in Canadian NHs. 
Methods: Leadership staff from a convenience sample of 37 NHs in 3 provinces ( $9 \mathrm{AB}, 10 \mathrm{MB}, 18 \mathrm{ON})$ completed a survey inquiring about 'when' ACP discussions occur, 'who' participates, 'what' medical choices are discussed, ready availability of prior ACP documents and adherence to prior decisions in medical crises. Data was analyzed using descriptive statistics.

Results: ACP discussions occur in $89 \%$ of homes upon admission, $92 \%$ with change in clinical status, and $76 \%$ yearly. Physicians always participated in $\mathrm{AB}$ homes, usually in $\mathrm{ON}$ homes, and virtually never in MB homes. Resuscitation, comfort care, hospital/emergency department transfers, antibiotics and artificial life support are always or almost always discussed in $86 \%, 83 \%, 81 \%, 80 \%$ and $62 \%$ respectively. ACP documents were available in $89 \%$ of homes, but adherence to prior decisions was only $64 \%$.

Discussion: There is substantial variability in ACP Discussion: timing and conduct, notably physician participation. Variability exists in which medical choices are discussed; resuscitation and comfort care are commonly discussed, whereas life support is discussed less.

Conclusion: Gaps remain in important processes of Advance Care Planning in Canadian nursing homes.

\section{What Matters: Voices of Older Adults 1.0 \& 2.0}

\section{Choi ${ }^{1}$, P. Jung ${ }^{2}$, M. Harder ${ }^{2}$, L. Styles ${ }^{2} .{ }^{1}$ Mount Royal University; ${ }^{2}$ Vancouver Island University.}

Background: The research purpose is to explore what matters most in everyday life to older adults living independently in the community, through giving participants a voice by asking them to share their perspectives.

Methods: This is a descriptive qualitative action research study, being undertaken in the emancipatory/critical paradigm (Polit \& Beck, 2017).

Results: Phase one (completed) of the project focused in on older adults living in Central Vancouver Island. A key output in phase one was the creation of a video that was screened to various audiences. The impact of screening the video in public venues with various audiences (e.g., older adults, informal caregivers, health students, etc.) resulted in (a) demystifying assumptions of aging, (b) opening up further dialogue about what matters to older adults, and (c) creating enhanced awareness amongst professional health care providers about the diversity of what matters to older adults (thus contributing to promoting stronger person-centered care approaches). These outcomes were consistent with the research paradigm of fostering change, as well as an important community-based knowledge mobilization approach.

Discussion: Considering the current and future ethnic diversity of the Canadian population, phase two expands the study to explore this central question with older persons of Chinese heritage living in British Columbia and Alberta. Research findings are intended to inform the general public, nurses and students to better understand what older adults' value and what is most important to them.

Conclusion: The research also gives voice to the older adults and can contribute to person-centered care of older adults.

\section{Assessing the Accuracy of International Classification of Diseases (ICD) Coding for Delirium}

\author{
V. Chuen ${ }^{1}$, A. Chan' ${ }^{2}$, V. Chau ${ }^{3}$, S. Alibhai ${ }^{3} .{ }^{1}$ McMaster \\ University; ${ }^{2}$ University of Toronto; ${ }^{3}$ University Health \\ Network.
}

Background: Delirium is common amongst hospitalized older adults and is associated with increased morbidity, longer lengths of stay, and higher costs. Following discharge, charts are coded according to the WHO International Classification of Diseases 10th edition (ICD-10) standards. The accuracy and completeness of these codes have implications for reimbursement, research and service programming. We assessed the accuracy of ICD coding for delirium and its relationship with delirium documentation in discharge summaries.

Methods: We performed a multi-centre retrospective chart review to identify hospitalized medical and surgical patients aged $\geq 65$ years with delirium using the Chart-based Delirium Identification Instrument (CHART-DEL). Administrative discharge abstract data were used to determine the presence of an ICD-10 code for delirium (F05). Charts of delirious patients were further divided based on whether "delirium" was documented in the discharge summary, and compared for the presence of a F05 code.

Results: Among delirious patients ( $\mathrm{n}=108)$, only $46 \%$ of charts received a F05 code. There was no difference in coding accuracy between medical and surgical patients ( $44 \%$ vs. $49 \%$ respectively, $\mathrm{p}>0.05$ ). The $\mathrm{F} 05$ code had a sensitivity of $46.3 \%$ and specificity of $100 \%$ for delirium, with a positive predictive value of $100 \%$ and a negative predictive value of $34.3 \%$. $67 \%$ of charts with "delirium" documented in the discharge summary $(\mathrm{n}=67)$ appropriately received a F05 code whereas only $12 \%$ of charts did when "delirium" was not documented.

Discussion: While the ICD-10 administrative code for delirium is highly specific, it cannot reliably identify delirium incidence given its poor sensitivity. Documentation of "delirium" in the discharge summary may contribute to improved coding accuracy.

Conclusion: Current ICD-10 administrative data inadequately captures delirium incidence. Further work is required to increase the accuracy of ICD coding for delirium.

Interface Geriatrics: Establishing a Novel Geriatric Rapid Access Clinic with One-Off CGA and Proactive Community Liaison 


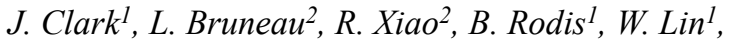
M. P. O'Connor ${ }^{l}$, A. Li ${ }^{1}$, A. Deshpande ${ }^{3}$, C. Shyr ${ }^{3}, A$. Cheung ${ }^{3}, N$. Varshney ${ }^{2}{ }^{1}$ University of British Columbia; ${ }^{2}$ Eagle Ridge Hospital; ${ }^{3}$ Fraser Health Authority.

Background: Repeat emergency department (ED) visits and hospital admissions are associated with adverse outcomes for geriatric patients and are costly to the healthcare system. In an effort to optimize care while making effective use of limited resources, we developed a Geriatric Medicine Rapid Access Clinic (RAC) for patients discharged from the ED.

Methods: As a single-centre initiative (five geriatricians, four geriatric nurses) from January 2019 to January 2020, community-dwelling older adults discharged from the ED were referred for a comprehensive geriatric assessment (CGA) within 1-4 weeks. Referrals were screened to include individuals who presented with acute functional decline, falls/ imbalance, polypharmacy, or multimorbidity. We compared the rate of ED re-visits and hospitalizations within a 28-day and 90-day period before and after RAC assessment.

Results: In a 28-day single-group pre-test post-test analysis, the rate of ED re-visits among 89 patients decreased $14.6 \%$ after RAC assessment and the rate of admissions decreased 5.6\%. A 90-day analysis among 69 individuals demonstrated an $11.6 \%$ decrease in ED rate of re-visits and $5.8 \%$ increase in admissions rate. A quasi-control study group indicated 14.5\% increase in admission rate for non-RAC assessed patients during the same period.

Discussion: In an older adult population of high ED utilization, this early data suggests that timely geriatric consultation post-ED discharge reduced the number of ED re-visits within both one and three months of assessment. Admissions may also be positively affected.

Conclusion: Interface Geriatric Medicine Rapid Access Clinics may be an effective strategy to reduce repeat ED visits among older adults.

\section{In Search of a Clinically Feasible Assessment Tool to Predict Dementia: Does Performance on a Dual- Task Gait Test Predict Future Cognitive Decline?}

\section{S. Cullen ${ }^{1}$, M. Borrie ${ }^{2}$, S. Carroll ${ }^{2}$, N. Kamkar ${ }^{3}$, N. Bray ${ }^{3}$, F. Pieruccini-Faria ${ }^{3}$, E. Figgins ${ }^{3}$, J. Mahon ${ }^{3}$, A. Osman ${ }^{3}, J$. Titus ${ }^{3}$, M. Montero-Odasso ${ }^{3} .{ }^{1}$ Western University, Department of Kinesiology; ${ }^{2}$ St. Joseph's Health Care, Parkwood Institute; ${ }^{3}$ Gait and Brain Lab, Parkwood Institute.}

Background: The numbers of cases of dementia are rising and there is an urgency to identify individuals at risk for dementia with clinically feasible assessment tools. Dual-task gait testing (walking while simultaneously performing a cognitively demanding task) is a test that shows promise. We aimed to determine if poor performance on dual-task gait testing is feasible to perform in a memory clinic and predictive of cognitive decline.

Methods: Every consecutive patient visiting a memory clinic in London, Ontario was assessed at baseline and 6 month follow-up from July 2015 to May 2019. At each visit, they performed a series of cognitive tests and a dual-task gait test (walking while counting). The magnitude of gait slowing due to the added cognitive demands, or the dual-task gait cost (DTC), was calculated as: ([usual gait velocity - dual-task gait velocity] / usual gait velocity) $\times 100$. Cognitive decline was ascertained by having significant decline on cognitive tests and by clinical consensus in subsequent visits. Cox models were used to evaluate the association between high DTC ( $>20 \%)$ at baseline and cognitive decline at follow-up.

Results: Eighty six patients (Mean age $=73.7$ years $[\mathrm{SD} \pm 9.5]$; $48.8 \%$ female) with at least one visit at 6 month follow-up were included. Fifty two (60\%) of them developed cognitive decline during follow-up. Cox models showed that patients with a DTC $>20 \%$ were at a significantly higher risk for future cognitive decline (hazard ratio $[\mathrm{HR}]=1.87,95 \% \mathrm{CI}=1.01$ $3.43, \mathrm{p}=0.046)$.

Discussion: Poor performance on dual-task gait testing, indicated by a high DTC, almost double the risk of future cognitive decline.

Conclusion: Dual-task gait testing is thus a feasible and promising tool in identifying patients at high risk for future cognitive decline.

\section{Artificial Intelligence, Therapeutic Relationship and Mental Health Care: Results from a Scoping Review with Focus on Dementia Care}

S. Dholakial, J. Gibson ${ }^{2}$, J. Shaw ${ }^{3}$, S. Benjamin 4 , J. Ho 4 . ${ }^{1}$ GeriMedRisk, McMaster University; ${ }^{2}$ University of Toronto;

${ }^{3}$ Women's College Research Institute; ${ }^{4}$ McMaster University.

Background: Therapeutic relationship in health care is a necessary and many a times, a sufficient platform to provide care to people with mental health needs. This platform traditionally has a human interface of a congruent therapist and a client in need, with implicitly normative boundaries of empathy, variable degree of physical/psychological contact and Trust. Artificial Intelligence (AI) and their applications in the field of mental health care have the potential to influence therapeutic relationship in unique yet unexplored ways. This presentation aims to answer the critical question: "What are the ways in which AI and its applications influence the field of therapeutic relationship (TR) in mental health care (MHC), especially in care for people with dementia"?

Methods: We adopt a validated scoping review methodology and locate academic literature on Medline, Embase, PsychInfo, SCOPUS, IEEE Xplore, Google Scholar, ACM (association of computer machinery) databases through an 
iteratively developed search strategy. We map the emerging themes around 84 included papers at the intersection of AI, TR and MHC. Furthermore, we analyze the themes around included literature at the intersection of TR, Dementia care and $\mathrm{AI}$ with an ethics of care lens approach.

Results: The analysis reveals multiple value level trade-offs at the intersection; maximizing utility vs. degree of deceit, and objective behavioral interactions as a trade-off for empathy were the central themes at this intersection.

Discussion: The results prompt the stakeholders involved in design, implementation, utilization and regulation of $\mathrm{AI}$ in the Dementia care field, to reflectively contemplate: "How is care getting defined at the intersection of AI, TR and Dementia care"?

Conclusion: This scoping review provides both, overarching insights and clues to operationalize changing boundaries of $\mathrm{TR}$ at this intersection of $\mathrm{AI}$ and dementia care.

\section{Care-of-the-Elderly Graduates Provide Essential Services in Alberta}

B. Dobbs, J. Triscott, L. Charles, P. G. Tian. Division of Care of the Elderly, Department of Family Medicine, University of Alberta,

Background: The Care of the Elderly (COE) Program is a 6-month to 1-year residency program on the care of older persons. This program was developed by the College of Family Physicians of Canada to address the increasing demand for health services among seniors. At the University of Alberta, the program has trained more than $60 \mathrm{COE}$ physicians since 1993. In 2019, we conducted a survey among the program's graduates to determine their practice characteristics, practice preparedness, and service provision.

Methods: This was an online survey (SurveyMonkey) in 2019 sent to all graduates of the COE Program. Descriptive statistics were used for analysis.

Results: There were 33 respondents with a response rate of $56 \%(33 / 59)$. The median age was 44 years (range: $30-66$ ), with $72 \%$ females $(24 / 33)$. With respect to practice, $48 \%$ of respondents (14/27) were full-time COE practice; $52 \%$ were part-time. Eighty-five point seven percent $(85.7 \%, 24 / 28)$ worked at least 31 hours/week, with 39\% (11/28) working more than 40 hours a week. COE practice occurred in various settings: office-based continuing care $(48.5 \%, 16 / 33)$, acute care consults $(48.5 \%, 16 / 33)$, acute care inpatients $(33.3 \%$, $11 / 33)$, home living $(27.3 \%, 9 / 33)$, supportive living $(22.4 \%$, $14 / 33$ ), and others. Remuneration for clinical work was predominantly fee-for-service $(60.6 \%, 20 / 33)$ and Clinical Alternative Relationship Plan reimbursement (39.4\%, 10/33). A significant number were also involved in teaching $(45.5 \%$, $15 / 33)$, administration $(36.4 \%, 12 / 33)$, and research $(30.3 \%$, $10 / 33)$. Close to seventy-six percent $(75.9 \%, 22 / 29)$ found the residency program very useful for practice, while $24.1 \%$ $(7 / 29)$ found it useful. The vast majority $(92.6 \%, 25 / 27)$ were satisfied with their professional life.

Discussion: COE graduates have integrated well into healthcare system.

Conclusion: The COE graduates provide clinical, academic, and administrative service in a variety of settings. As the older population increases, the need for COE physicians increases as well.

\section{Association Between Maladaptive Obstacle Negotiation and Fall Risk in Mild Cognitive Impairment}

F. Faria-Pieruccini ${ }^{1}$, S. Cullen ${ }^{2}$, N. Bray ${ }^{2}$, Y. SarquisAdamson ${ }^{2}{ }^{1}$ Western University, Department of Medicine; ${ }^{2}$ Gait and Brain Lab, Parkwood Institute.

Background: Individuals with Mild Cognitive Impairment (MCI) have reduced anticipatory gait adjustments to avoid a collision with an obstacle while walking and talking compared to controls. This maladaptive motor behaviour could be a risk factor for falls in MCI. The aim of this study is to test the hypothesis that reduced anticipatory adjustments are associated with history of recent falls in MCI.

Methods: A total of 73 older individuals diagnosed with amnestic MCI from the Gait and Brain Study cohort were analyzed. Participants' walking performance was cross-sectionaly tested during two conditions: i) approaching and stepping over an obstacle (single task); ii) approaching and stepping over an obstacle while counting backwards by $1 \mathrm{~s}$ from 100 (dual task). Preparatory step-to-step length adjustments (i.e. length variability) to avoid hitting an "ad hoc" obstacle, set at $15 \%$ of participant's height, were electronically measured during pre-crossing phases: early ( 3 steps before the late phase) and late (3 steps before obstacle). The variability increase from early to late phase identified who and who did not perform anticipatory adjustments. Falls in the previous 12 months were recorded with falls calendar. Binary logistic regressions tested associations between anticipatory adjustment and falls history adjusted for potential confounders.

Results: Individuals with MCI who did not perform anticipatory adjustments while approaching the obstacle during dual task condition had higher risk of have suffered at least one recent fall compared to those who adjusted (adjusted OddsRatio 4.98 95\%CI 1.47-16.89; $\mathrm{p}=.01$ ).

Discussion: The additional cognitive complexity for some individuals with MCI may affect the adaptive control of their locomotion making them more vulnerable to collisions.

Conclusion: Reduced anticipatory adjustment to avoid a collision with obstacle is a fall risk factor in MCI. 
Characterizing the Relationship Between Responsive Behaviours and Duration of Stay in ALC Patients

J. Flores ${ }^{1}$, A. Dominic ${ }^{2}$, G. Blake ${ }^{2}$, Y. Henderson-Haars ${ }^{2}$, M. Lui ${ }^{2} .{ }^{1}$ University of Toronto; ${ }^{2}$ Hamilton Health Sciences.

Background: Alternative level of care (ALC) patients are those who do not require the intensity of care provided by acute care hospitals but cannot access the appropriate healthcare resources post-admission to make their discharge safe. The main objective of our study was to determine whether responsive behaviours are independently correlated with duration of stay in ALC patients.

Methods: A retrospective chart audit was conducted on 110 patients in the ALC ward at Juravinski Hospital in Hamilton. Patients assigned ALC status were eligible for the study. The charts were reviewed for the total duration of responsive behaviours, age, sex, duration of stay, and the presence of central nervous system (CNS)-altering medications, among others.

Results: The median age of patients included in the study was 85. The total duration of responsive behaviours was found to be correlated with duration of stay in ALC patients. Sex was found to be an effect modifier that influenced the relationship between responsive behaviours and ALC stay. For females, every 10 additional days of responsive behaviours increased the duration of ALC stay by $1.3 \%$. For males, every 10 additional days of responsive behaviours increased the duration of ALC stay by $41.2 \%$. The presence of CNS-altering medications was not correlated with a longer duration of stay.

Discussion: Reducing responsive behaviours, especially in males, should theoretically lower duration of stay in ALC patients. Female responsive behaviours were usually described as irritable whereas male responsive behaviours were usually described as aggressive or violent.

Conclusion: Responsive behaviours were found to be correlated with duration of stay in ALC patients, with sex found to be an effect modifier. This study provides evidence that could enable tertiary hospitals to better care for this population.

\section{Health-Seeking Behavior Related to Selected Dimensions of Wellness in Community-Dwelling Older Adults}

N. Gill, D. Connelly. University of Western Ontario.

Background: Older people generally prefer to 'stay-put' in their own homes. Informed by the Seven Dimensions of Wellness, a component of the International Council on Active Ageing (ICAA) Model, measures of physical function (i.e. physical), fall risk (i.e. environmental), and psychosocial factors (i.e. emotional, spiritual and social) related to health have been selected with the assumption that they intersect to influence health-seeking behaviour of older adults ageing-at-home.
The findings are anticipated to increase our understanding of the relationship(s) among health-seeking behaviour, physical function, fall risk, resilience, social and spiritual well-being, and awareness of available services in a group of communityliving older adults aiming to age at home.

Methods: The objective of the study is to investigate the relationship among dimensions of well-being, including physical function, fall risk, psychosocial factors and awareness of community-support services, with health-seeking behaviour in community-dwelling older adults. This project will use a correlational design. A sample size of 98 subjects will be recruited. Subjects will be older adults living independently at home in the city of London, ON, aged $\geq 65$ years, ambulatory (with/without gait aid) and without executive function impairment. Data will be analyzed using multiple linear regression.

Results: The study is still in the data collection phase

Discussion: The present study aims to explore if awareness of certain factors related to ageing at home successfully may predict the health-seeking behaviour of older adults along with expanding the literature about the health-seeking behaviour of community-living older people. The resulting information may assist and/or guide the efforts to better help older adults age-in-place and access community support services.

Conclusion: The study is still in the data collection phase.

\section{Older Adults' Perceptions of Frailty}

S. Guo ${ }^{1}$, D. Rolfson ${ }^{1}$, H. Hansen ${ }^{2}$, S. Khera ${ }^{1} .{ }^{1}$ University of Alberta; ${ }^{2}$ University of Calgary.

Background: As one of the geriatric giants, frailty is a state of decreased physiological reserve and increased vulnerability that results from the collective decline in multiple systems. It manifests as a heterogeneous, multidimensional syndrome. However, several studies have shown that older adults associate many negative aging stereotypes with the term frailty. This study aims to describe how acceptable and useful the term frailty is as viewed by a mixed group of older adults.

Methods: In this qualitative study, we completed 23 interviews with participants aged 65 or older and of a variety of fitness and frailty. We designed semi-structured interviews to explore participants' perceptions of the term frailty and the individual components of the medical concept of frailty, as well as perceived utility of the concept of frailty. The Edmonton Frail Scale (EFS) is being used to assess the frailty status of each participant. We are employing interpretive description methodology to qualitatively analyze the interview data, including comparisons based on respondent groups (i.e. frail vs. non-frail).

Results: The study is still within the analysis phase. Preliminary results suggest broad themes including 1) association of the word frailty with negative social connotations and lack of frail self-identification, 2) emphasis of themes where 
there is personal experience, and 3) despite negative views of frailty and initial negative emotions, some older adults still find importance in the disclosure if there are actionable items afterwards.

Discussion: Preliminary results suggest although there are negative connotations to the disclosure of frailty status, older adults may still find the disclosure useful if there are follow up clinical recommendations.

Conclusion: This study will describe the relative acceptability and perceived utility of the term and concept of frailty amongst older adults.

\section{Environmental Scan to Identify Community-Based Models of Dementia Care that Improve Quality of Life for Persons Living with Dementia and Their Care Partners}

\section{Helmer-Smith ${ }^{1}$ L. Crowe ${ }^{1}$, D. Houghton, J. LaPlante, T. MacDonald ${ }^{2}$ J. Major, D. Mastin, I. Moroz ${ }^{1}$, L. Poole ${ }^{3}$, M. B. Wighton ${ }^{3}$, C. Liddy ${ }^{4} .{ }^{1}$ C.T. Lamont Primary Health Care Research Centre, Department of Family Medicine, University of Ottawa; Bruyère Research Institute; ${ }^{2}$ Canadian Foundation for Healthcare Improvement; ${ }^{3}$ Dementia Advocacy Canada; ${ }^{4}$ C.T. Lamont Primary Health Care Research Centre, Department of Family Medicine, University of Ottawa; Bruyère Research Institute; Ontario eConsult Centre of Excellence, The Ottawa Hospital.}

Background: Dementia is a growing concern in Canada, with implications for the health of Canadians and costs for future care. The key objective of this environmental scan was to identify published community-based models of dementia care, strategies, and policies supporting the implementation of these models', and to examine their effectiveness in improving quality of life.

Methods: This study was implemented in three parts: 1) academic literature search in five databases, 2) grey literature search of existing dementia strategies and programs across Canada, as referenced in the National Dementia Strategy, and 3) weekly working group meetings to identify additional resources; discuss gaps in services and models; and inform scoping needs.

Results: Of the 901 articles retrieved during the literature search, 26 were included in our review. Three types of approaches to dementia care at the primary care level were identified: screening tools, primary care-based collaborative models, and educational interventions. A lack of programs and jurisdictional dementia strategies were identified in Yukon and Nunavut. Between July and September 2019, the working group identified 19 additional resources worthy of further analysis regarding effectiveness to improve quality of life (research networks, models of care, and formal recommendations).
Discussion: Most articles featured multi-component collaborative models of care, characterized by a team-based approach, having a care/case manager, individualized care plans, stepped care approach, and consideration for care partners' needs. Given primary care practices are complex adaptive systems, no single model fits the context of every practice. The evidence recommends that the core components be adapted to local needs, complexities, and resource constraints.

Conclusion: This study identified five common core components of primary care-based models of dementia care that improve quality of life for persons living with dementia and their care partners.

\section{The Feasibility of Using eConsult in Long-Term Care Facilities*}

M. Helmer-Smith ${ }^{1}$, C. Fung ${ }^{2}$, A. Afkham ${ }^{3}$, L. Crowe ${ }^{l}$, M. Gazarin ${ }^{4}$, E. Keely ${ }^{5}$, I. Moroz ${ }^{1}$, C. Liddy ${ }^{6} .{ }^{\text {I C.T. Lamont }}$ Primary Health Care Research Centre, Department of Family Medicine, University of Ottawa; Bruyère Research Institute; ${ }^{2}$ St. Patrick's Home of Ottawa; Department of Family Medicine, University of Ottawa; ${ }^{3}$ Champlain Local Health Integration Network; ${ }^{4}$ Centre of Excellence for Rural Health and Education, Winchester District Memorial Hospital; ${ }^{5}$ Department of Medicine, University of Ottawa; Division of Endocrinology/Metabolism, The Ottawa Hospital; Ontario eConsult Centre of Excellence, The Ottawa Hospital; ${ }^{6}$ C.T. Lamont Primary Health Care Research Centre, Department of Family Medicine, University of Ottawa; Bruyère Research Institute; Ontario eConsult Centre of Excellence, The Ottawa Hospital.

Background: Residents of Canada's long-term care (LTC) homes often face multiple chronic conditions and require above-average need for specialty care. These same conditions make travel to appointments extremely challenging. Electronic consultation (eConsult), a secure web-based tool that allows patient-specific, asynchronous communication between primary care providers (PCPs) and specialists, has demonstrated ability to improve access to specialist advice in primary care and for older adults. Despite its potential, little research has been done to assess feasibility and impact in LTC settings.

Methods: We conducted a cross-sectional study of eConsults submitted by LTC PCPs in Eastern Ontario between January 1 and December 31, 2018. Routine utilization data was collected and descriptive statistics were calculated. We completed thematic analysis of four focus groups with LTC early adopters.

Results: 64 cases were submitted to 23 specialty groups, most frequently dermatology (19\%), geriatric medicine (11\%), and infectious disease $(9 \%)$. Specialists responded in a median of 0.6 days. $70 \%$ of cases were resolved without the resident needing a face-to-face specialist visit. In $60 \%$ of cases, PCPs received advice for a new or additional course of action. 


\section{BACSU: FAMILY PHYSICIANS AND DEMENTIA CARE}

Participants described LTC homes as under-resourced, with limited IT and clerical support. They recognized eConsult is valuable in LTC and identified considerations for implementation (i.e. clinician champion, use of delegates, and workflow integration).

Discussion: Our results align with studies conducted in primary care. eConsult improves access to specialist advice, reduces costs, and increases ability to provide care that aligns with the resident's goals. Implementation of eConsult in Ontario's LTC homes is continuing, informed by the complexities and considerations identified by participants.

Conclusion: eConsult is feasible in LTC homes and should be used across Canada to improve equity of access to specialist advice.

*This study is reported in full in the Journal of the American Medical Directors Association.

\section{The Pearly Bird Gets the Worm: A Qualitative and Quantitative Analysis of the Geriatrics Update: Clinical Pearls conference}

\author{
P. Hoang ${ }^{1}$, L. Torbiak ${ }^{2}$, Z. Goodarzi ${ }^{1}$, H. Schmaltz ${ }^{1}$ \\ (Primary Investigator). ${ }^{1}$ University of Calgary; ${ }^{2}$ University \\ of Manitoba.
}

Background: Geriatric Continuing Medical Education (CME) for family physicians (FPs) is needed given increasing population age and complexity. We formally evaluated the University of Calgary Cumming School of Medicine Annual Geriatrics Update: Clinical Pearls Course (Geriatrics Update) as one model to address primary care learning needs in geriatrics.

Purpose: To identify: 1)How this one-day course meets participant FPs' perceived learning needs, and 2)Additional modifications to better fulfill learning needs and create practice changes.

Methods: Multi-method approach-descriptive statistics from 2018-2019 Geriatrics Update evaluation surveys, and thematic analysis of semi-structured FP telephone interviews.

Results: All $(\mathrm{n}=20)$ interviewees over 2 years described fulfilled learning needs and planned to return. The Geriatrics Update course is the primary source of Geriatric CME for $60 \%$ of interviewees. $10 \%$ of interviewees denied major deficiencies (2018), compared to 70\% (2019). Themes included: highly relevant, convenient, and valuable given complex patient population. Common requests included: more short snappers (2018), and more practical clinical resources/tools and pearls $(2018,2019) .60 \%(n=90 ; 2018)$ and $45 \%(n=64$; 2019 ) of participants completed the evaluation ( $58 \%$ and $63 \%$ FP respondents, respectively). 85\% (2018) and 73\% (2019) of survey respondents intended to make practice changes.

Discussion: Course objectives continued to show significant before-after improvements in confidence $(\mathrm{p}=0.00)$ in 2018/2019. Logistical timing issues in 2019 affected both survey and interview evaluations with frequent requests to better balance content, discussion, and networking time. Study limitations include selection bias in survey respondents and interviewees.

Conclusion: Iterative evaluation of Geriatrics Update confirmed that the course is well received and often the primary source of geriatric CME for FP's. Interviews provided additional context and descriptive feedback to improve course delivery and better meet FP learning needs.

\section{Use of Medium Chain Triglyceride (MCT) Oil in Subjects with Alzheimer's Disease: A Randomized Double-Blind Placebo Cross Over Study, with an Open Label Extension}

\author{
A. Juby, D. Mager, T. Blackburn, C. Davis, D. Jay. \\ University of Alberta.
}

Background: Cerebral glucose and insulin metabolism is impaired in Alzheimer's disease (AD). With low glucose availability the brain can also use ketones as an alternative energy source (usually from stored triglycerides in fasting/ low calorie intake states). MCTs are an exogenous (dietary) source of ketones and have been well studied in the field of parenteral nutrition. Attention recently is in their use as a source of cerebral ketones for AD subjects. The objective of this study is to evaluate the impact, if any, of MCT oil on cognition, activities of daily living (ADLs) and behavior in AD subjects.

Methods: Six month randomised, double blind, placebo, cross over study, with 6 month open label extension evaluating MCT oil versus olive oil in cognition, behavior and ADL function in AD subjects. Subjects could continue cholinesterase inhibitors, memantine and antidepressants provided the doses remained stable during the study. Approved by the Health Canada and local HREB.

Results: Twenty community dwelling AD subjects completed the first 6 months, 19 completed the full 15 months. Participants were aged 54-84yrs (average 72.6yrs), 11 men and 9 women. $70 \%$ had College/University education. Baseline MMSE 22.6/30 (10-29), and MoCA 15.6/30 (4-30). Baseline Cognigram ${ }^{\circledR}$ Part 1 ranged from 65-106, Part 2 from 48-107. Average MCT oil consumption was 1.8 tablespoons daily $(25.2 \mathrm{~g}, 234 \mathrm{kcal})$. There was individual variability in MMSE and MoCA over the course of the study, but most $(80 \%)$ remained stable or improved. Lower homocysteine resulted in higher final MoCA ( $\mathrm{p}=0.03)$.

Discussion: Longer MCT exposure and age $>73$, resulted in higher final MMSE $(\mathrm{p}<0.001)$ and Cognigram 1.

Conclusion: $80 \%$ showed stabilization or improvement in cognition, with better response in those on 9 months of continual MCT oil. 


\section{Creutzfeld-Jacob Disease: Will You Know It When You See It?}

\author{
S-L. Kane ${ }^{1}$, R. D'Amour ${ }^{2} .{ }^{1}$ Western University; \\ ${ }^{2}$ Creutzfeldt-Jakob Disease Surveillance System, Public \\ Health Agency of Canada.
}

Background: Creutzfeld-Jakob disease (CJD) is a rare cause of dementia with only 79 confirmed cases in Canada in 2018. This dementia can present to a variety of clinicians. It is important the clinicians are familiar with the diagnostic criteria, which were updated by the Centre for Disease Control in 2018.

Methods: Using two autopsy confirmed cases of CJD this review will compare two different presentations.

Results: These two cases illustrate that the temporal course can be quite varied. Early presentation consisted of ataxia, apraxia, and extra-pyramidal features. Myoclonic jerks presented in the middle stage, while mutism was a late feature. Pyramidal features of visual spatial difficulties (case1) and ideational and ideomotor apraxia occurred in both cases in the early to mid-stage. Positive MRI findings occurred in only one case. CSF protein testing was not possible in either case.

Discussion: Literature shows neuropsychiatric and cognitive symptoms, with impaired attention and amnesia most frequently for all subtypes (J Alzheimer Dis. 2017;59:329). These were not an early feature of either case. Both were referred to Neurology for balance difficulties prior to Geriatrics for cognitive difficulties. The duration of disease was longer than usual. The clue to CJD was the marked increase in tone early in the dementia with extrapyramidal, pyramidal, and cerebellar findings. Myoclonus was present in both cases, but not early enough to obtain appropriate ante mortem testing. Families were notified of the possibility of CJD and autopsy discussion occurred prior to death. The CJD Surveillance System and Public Health were notified in advance for safety of funeral home and pathology staff.

Conclusion: CJD may present to a variety of clinicians. The 2018 CDC criteria were not helpful to obtain full testing early in the disease. This diagnosis requires a high index of suspicion. The CJD Surveillance System's Intake Form is a helpful guide and should be known to clinicians seeing older adults.

\section{Acute Psychosis Episode as the Initial Presentation of Dementia with Lewy Body: A Case Report}

\section{K. S. Karpageshwary', S. Stacey'. ' Ministry of Health Holdings; Sengkang General Hospital; ${ }^{2}$ Sengkang General Hospital.}

Background: Dementia with Lewy bodies (DLB) is the second most common cause of neurodegenerative dementia. When presenting with psychosis, it is prudent to investigate for underlying medical conditions which could explain the presentation and obtain a detailed history to determine if an underlying neurodegenerative disorder is plausible.
Methods: A 79-year-old male was referred from the Institute of Mental Health for evaluation of his acute onset psychotic episode. Patient was convinced that terrorists were present to harm him in his home and was found armed with a knife for self-defence. Initial differentials were that of delirium, intracranial pathologies and encephalopathies including that of autoimmune and metabolic aetiology. Physical examination did not yield rigidity or focal neurological deficits. Haematological investigations revealed inflammatory markers within normal limits with nil electrolytes imbalances. Magnetic resonance imaging (MRI) of the brain was negative for a space occupying lesion or changes consistent with encephalitis.

Results: He was subsequently initiated on quetiapine to address his aggressive behaviour. Corroborative history from family revealed that the presence of persistent recurrent visual hallucinations for the past 1 year including images of snakes, children and terrorists. In conjunction there was difficulties with sleep, presence of multiple night time awakening with motor disturbances, and short-term memory impairment. During inpatient stay fluctuations in cognitive state was observed independent of medication intervention. Cognitive assessment revealed Mini Mental State Examination (MMSE) 26/28, Montreal Cognitive Assessment 26/20 and Frontal Assessment Battery (FAB) 12/18.

Discussion: Diagnosis of DLB presenting with a brief psychotic episode was made; meeting criteria with vivid visual hallucinations, Rapid Eye Movement (REM) sleep disorder and fluctuating state in the Background: of cognitive impairment and delusion misidentification syndrome.

Conclusion: Patient was discharged with oral quetiapine and rivastigmine patch.

\section{Cannabis Use Among Middle and Older} Aged Adults Prior to Legalization in Canada: A Gendered Analysis of the Canadian Tobacco, Alcohol and Drugs Survey 2017

\section{A. Keethakumar, V. Mehra, N. Khanlou, H. Tamim. York University.}

Background: The recreational use of cannabis was legalized across Canada in October 2018. While many use cannabis without harm, adverse outcomes have been noted in older adults. Given the lack of literature on cannabis use among this population, the objective of our study was to identify the prevalence and characteristics of cannabis use among middle and older-aged adults prior to legalization.

Methods: Secondary analysis was conducted on the Canadian Tobacco, Alcohol and Drugs Survey 2017 and sample was restricted to adults aged 40 years and older. Analysis was conducted separately for males and females and outcome defined as past-year cannabis use. Bivariate and multivariable logistic regression was performed to identify associations between sociodemographic, health, and substance use variables, and the main outcome. 
Results: In 2017, middle and older aged females reported $5.9 \%$, while males reported $9.0 \%$ prevalence of past-year cannabis use. Significant predictors of the outcome for females included residing in urban communities (OR: 2.21, 95\% CI: 1.24-3.91), British Columbia (OR: 5.50, 95\% CI: 2.4-12.8) or the Eastern-Atlantic provinces (OR: 2.63, 95\% CI: 1.4-4.9), having fair/poor mental health (OR: 4.29, 95\% CI: 1.1-16.3), smoking cigarettes (OR: $2.90,95 \%$ CI: 1.5-5.6), and drug use (OR: 6.99, 95\% CI: 1.2-41.8). Significant predictors for males included having no partner (OR: 1.67, 95\%CI: 1.1-2.6), smoking cigarettes (OR: 4.51, 95\% CI: 2.5-8.2), and drug use (OR: 40.40, 95\% CI: 10.0-162.6).

Discussion: Our findings show that cannabis use varied between the sexes and is unlike other age groups. Results from this study can be used to track changes in cannabis-related behaviours over time.

Conclusion: This is one of the first nationally representative studies to outline the characteristics of cannabis use in middle and older-aged Canadians before legalization.

\section{Primary Care Nutrition for Older Adults: Feasible Care Processes and Approaches for Canada}

H. Kellerl, C. Chan ${ }^{2}$, C. Laur ${ }^{3}$, R. Donnelly ${ }^{4}{ }^{1}$ Schlegel-

University of Waterloo Research Institute for Aging;

${ }^{2}$ University of Alberta; ${ }^{3}$ Women's College Hospital;

${ }^{4}$ University of Waterloo.

Background: Nutrition risk occurs in about $34 \%$ of community-living older adults in Canada and is associated with hospitalization and mortality. Nutrition risk is also often comorbid with frailty. As we work towards identifying and supporting pre-frail individuals, nutrition is a logical component of geriatric care. The purpose of this study was to develop a feasible nutrition care pathway for primary care based on experiential and empirical evidence.

Methods: An environmental scan of guidelines from leading national and international nutrition organizations, and a literature review (PubMed and CINAHL 2009-2019) was completed. A draft pathway was reviewed by a national group of health care providers (e.g. dietitians, physicians, nurses) who participated in a one-day think tank $(n=22)$ and three follow-up teleconferences. A subsequent near-final version was vetted by a regional group that included diverse researchers, health and community service providers $(\mathrm{n}=38)$.

Results: The environmental scan ( $\mathrm{n}=128$ publications) identified risk screening, referral to a dietitian, community services, and monitoring as key steps in a nutrition pathway for older adults. No existing nutrition pathway for primary care was found. Think tank participants discussed these care activities, how they could be connected, and what resources were required to ensure feasibility in their setting. Community service providers further noted how to promote feasibility in diverse contexts.
Discussion: The development of a nutrition care pathway is the first step to systematically promoting the prevention, detection, and treatment of malnutrition among older adults living in the community. The pathway created considers the diversity of primary care resources and experiences for most of Canada.

Conclusion: Further vetting of this pathway is underway with an online survey open to patients/advocates as well as primary care and community service providers

\section{Food Security and Health Status of Indigenous Seniors in Northern Canada}

K. Kerber, W. Gibson, F. Kolahdooz, S. J. Lang, J. McKeen, M. Laboucan, S. Irlbacher-Fox, K. Kandola, A. Corriveau, A. Wagg, S. Sharma. University of Alberta.

Background: Indigenous seniors living in Northwest Territories (NT) have experienced a rapid transition away from a traditional lifestyle, and are at higher risk for diet-related chronic disease. Indigenous seniors may also experience food insecurity due to limited access to traditional foods, higher food prices and limited fresh food related to geographic remoteness.

Methods: We undertook a mixed-methods study on diet, health status, and access to healthcare among Indigenous seniors $(50+$ years) in Inuvik, NT. Data were collected via one-on-one interviews $(\mathrm{n}=71)$, sharing circles with caregivers and Elders $(\mathrm{n}=4)$, and key informant interviews $(\mathrm{n}=12)$ and included questions about food security, nutrition, self-reported health conditions, and access to healthcare.

Results: Nearly one in five (17\%) participants reported moderate to severe food insecurity and almost half reported eating alone in the past month (45\%), and a third reported eating alone sometimes (35\%). Participants expressed concern about the availability of traditional foods for Elders in residential or hospital care. Two-thirds (64\%) of participants reported being in good, very good, or excellent health but $81 \%$ reported at least one health condition, and $46 \%$ reported two or more conditions.

Discussion: Access to traditional foods and social connectedness are critical components of health among Indigenous peoples. Indigenous seniors experience food insecurity, particularly regarding access to traditional foods. Participants reported fairly high self-rated health, however most were living with at least one comorbid condition.

Conclusion: The next phase of the project will include sharing results with communities and stakeholders and the development of an Elder-led community wellness program. Future studies will be guided by the community to further understand and mitigate the impact of dietary transition among Indigenous seniors.

A Qualitative Study of Health Care Service Use Across Phases of Alzheimer's Disease Caregiving 
K. Kokorelias ${ }^{1}$, M. Gignac ${ }^{2}$, G. Naglie ${ }^{3}$, N. Rittenberg ${ }^{1}$, J. Mackenzie ${ }^{4}$, S. De 'Souza ${ }^{5}$, J. Cameron ${ }^{1}$. ${ }^{1}$ University of Toronto; ${ }^{2}$ Institute of Work and Health; ${ }^{3}$ Baycrest Health Sciences; ${ }^{4}$ Ontario Shores; ${ }^{5}$ Providence Health.

Background: Caregiving is not static and changes across the illness trajectory. Understanding caregiving phases allows the provision of timely supports and services to maximize caregiver and care recipient quality of life. Our first research objective was to determine phases of caregiving throughout the Alzheimer's disease trajectory. Our second objective was to explore service use decision-making across these caregiving phases.

Methods: We applied a constructivist grounded theory approach to data collection and analysis. Forty spousal (10 husbands +10 wives $)$ and adult children ( 10 sons +10 daughters) caregivers to persons with Alzheimer's disease were recruited and interviewed using a semi-structured interview guide.

Results: We identified 5 phases reflecting caregiving roles related to: 1) monitoring initial symptoms, 2) navigating diagnosis, 3) assisting with instrumental activities of daily living, 4) assisting with basic activities of daily living, and 5) preparing for the future. Service use was influenced by two key factors that evolve over the caregiving phases: the goals of caregiving and the practicalities of accessing services. Caregivers select services to meet their care goals as they evolve across phases of caregiving. Practical factors also influence the decision-making processes and are often related to personal, family, and/or external factors that relate to the feasibility of obtaining a certain service. We were not able to identify any differences by gender or relationship.

Discussion: We identified five distinct phases of caregiving and factors influencing service use decision making across the Alzheimer's disease trajectory. Caregiver decision-making regarding service use remains contextual to the changes in the caregiving and illness trajectories.

Conclusion: Findings can be used to inform the development, evaluation, and implementation of services to meet the changing needs of dementia caregivers across the illness trajectory.

\section{Optimising Inappropriate Medication Use Among Long-Term-Care Residents with Advanced Dementia-Results from the Optimamed-LTC Study}

E. Kröger, J. Plante, M. Morin, P. Voyer, P-H. Carmichael, N. Champoux, J. Monette, A. Giguèrer, M. Aubin, P. Durand, M. Wilchesky. Université Laval, Centre d'excellence sur le vieillissement de Québec, Université Laval, Centre d'excellence sur le vieillissement de Québec, Université Laval, Centre d'excellence sur le vieillissement de Québec, Université Laval, Centre d'excellence sur le vieillissement de Québec, Centre d'excellence sur le vieillissement de Québec, CIUSSSCN, Université de
Montréal, McGill University, Laval, Université Laval, Centre d'excellence sur le vieillissement de Québec, Université Laval, Centre d'excellence sur le vieillissement de Québec, McGill University, Donald Berman Maimonides Geriatric Centre.

Background: Long-term care (LTC) residents with severe dementia receive multiple medications. With disease progression, a palliative approach should be considered. Accordingly, medications should be reviewed, and deprescribed if necessary. The aim of this controlled quasi experimental trial was to evaluate the effect of an interprofessional knowledge exchange (KE) intervention to decrease use of medications of questionable benefit among LTC residents.

Methods: A 6-month intervention was implemented in 4 LTC in the greater Quebec City health board, while 3 comparable LTCs served as controls. Published lists of « mostly ", " sometimes » or " exceptionally » appropriate medications, tailored for LTC facilities were used. The intervention consisted in: 1) information for participants' families about optimal medication use in severe dementia; 2) participation of facilities' nurses, pharmacists and physicians in a 90-minutes KE session; 3) medication review by pharmacists for each participant using the tailored lists; 4) recommended changes after discussions with nurses or physicians. Agitation and pain levels of participants were evaluated using the CMAI and PACSLAC scales.

Results: Eighty-three of 120 eligible residents could be followed-up. The mean number of regular medications per participant decreased from 8.0 to 7.6 in the intervention group and from 10.6 to 9.0 in the control group (p-value for the difference in differences test: 0.09). Levels of agitation and comfort did not change noticeably during follow-up.

Discussion: Practice changes within all facilities of the health board and a ceiling effect in the intervention facilities may have prevented a significant effect of the intervention.

Conclusion: This interdisciplinary intervention to optimize inappropriate medication use in LTC residents resulted in decreased mean numbers of medications per resident after 6 months, in both intervention and control LTC facilities.

\section{Co-Creation and Evaluation of a "Sustain and Gain" Falls Prevention Maintenance Program for Community-Dwelling Older Adults in Greater Sudbury}

B. Lafleurl, J-A. Clarke', S. Jibb², S. Gingrich ${ }^{2}$, S. Lane ${ }^{2}$. ${ }^{I}$ North East Specialized Geriatric Centre; ${ }^{2}$ Health Sciences North-North East Specialized Geriatric Centre.

Background: Group-based fall-prevention exercise programs reduce the rate of falls amongst community-dwelling older adults. Participants in our local falls program improved their balance performance upon completion, but these benefits 
were lost after a 6-month follow-up. The goal of this study is to test the efficiency of 'Sustain and Gain', a pilot program designed to continue the improvement in balance performance in graduates of the fall program.

Methods: The 'Sustain and Gain' program was developed in two phases. Phase I (developing the maintenance program) used an experienced-based co-design that included 11 participants, their care partners and members of the research team. Phase II (implementation and evaluation) used a mixed methodology that included: quantitative analysis to measure the participants' physical outcomes (Timed Up and Go, 2-minute walk test, Berg Balance Scale) and quality of life (Older Peoples' Quality of Life Questionnaire) measured at baseline, 3 months, 6 months, and 9 months, as well as a qualitative analysis to gain a deeper understanding of participants' experience and their satisfaction with the program.

Results: Preliminary results of 18 participants demonstrate an overall improvement in balance performance, and initial qualitative analyses demonstrate positive feedback about the program. Implementation of the program was completed on Dec 31st 2019 and final results will be available for the presentation/poster.

Discussion: The 'Sustain and Gain' program may continue the improvement of balance performance of frail older adults. The positive social and psychological outcomes expressed by the participants further support the benefit of the program.

Conclusion: Preliminary findings from the 'Sustain and Gain' program support the need for an ongoing fall-prevention program in community-dwelling older adults.

\section{Does Exercise Reduce Obstructive Sleep Apnea Severity in Elderly Patients? A Systematic Review}

H. L. Lam ${ }^{1}$, W. T. V. Li ${ }^{1}$, I. Laher ${ }^{2}$. ${ }^{1}$ University of Hong Kong, ${ }^{2}$ University of British Columbia.

Background: The prevalence of sleep apnea increases with age, peaking at the age of 60 . Current standard of treatment (CPAP) fails to reduce cardiovascular outcomes and is limited by very poor compliance and tolerability. Exercise can reduce sleep apnea severity. We analysed the efficacy of physical exercise on sleep apnea patients aged 60 years or above.

Methods: Two independent reviewers searched MEDLINE, EMBASE, CINAHL, CENTRAL and ClinicalTrials.gov databases for trials, reported up to 30th November 2019, that measured pre and post intervention apnea hypopnea index (AHI) with exercise as the primary intervention. We contacted study authors for individual patient data in studies that also included patients younger than 60 years old. Body mass index (BMI) was studied as a secondary outcome.

Results: 5 trials (4 randomised controlled trials, 1 nonrandomised interventional trial) with a total of 98 participants were included. The combined reduction in AHI due to exercise was -6.884 events/hour (95\% CI -8.454 - -5.314, $\mathrm{p}<0.005)$. Relative risk and odds ratio did not show a reduction in $\mathrm{AHI}$ in patients who exercised. (RR: 1.352, 95\% CI 0.992-1.843, OR: $2.232,95 \%$ CI $0.982-5.076)$. In 3 trials with 75 participants, the change in BMI was not significant. $\left(0.024 \mathrm{~kg} / \mathrm{m}^{2}, 95 \% \mathrm{CI}\right.$ $-2.293-2.340, \mathrm{p}=0.984$ )

Discussion: Limitations of the study include a small sample size and selection bias due to exclusion of 6 studies where we could not obtain patient data. More studies are needed to investigate the effect of exercise on cardiovascular outcomes in elderly OSA patients.

Conclusion: Exercise as the sole intervention reduced sleep apnea severity in patients 60 or above, without significant changes in BMI.

\section{Creating a Framework for a New Division of Care of the Elderly within the Department of Family and Community Medicine at the University of Toronto}

E. Lass ${ }^{1}$, D. Tannenbaum ${ }^{2}$, S. Feldman ${ }^{3} .{ }^{1}$ University of Toronto; ${ }^{2}$ Department of Family and Community Medicine, Mount Sinai Hospital, University of Toronto; ${ }^{3}$ Department of Family and Community Medicine, Baycrest Health Sciences, University of Toronto.

Background: With Canada's rapidly aging population, there is a critical need for all family physicians to be competent in caring for older adults. At the University of Toronto Department of Family and Community Medicine (DFCM), there is currently no Division of Care of the Elderly (DCOE). In Canada, there are two existing formal DCOEs within family medicine departments. The project goal for the DCOE is to support all faculty in providing better teaching and care for older adults and support care of the elderly physicians at the DFCM.

Methods: Thirty-two semi-structured stakeholder interviews were conducted between November 20, 2018 and January 8, 2020 with stakeholders at the University of Toronto, leadership in the two current Canadian Family Medicine DCOEs, and patient advisors. Common themes were identified, and results were collated into a SWOT analysis.

Results: Diverse opinions were gained from leaders in geriatric medicine, geriatric psychiatry, family physicians, and patient participants. Opportunities for a DCOE included increased academic productivity, greater interprofessional and interdepartmental collaboration, enhanced faculty development and trainee education, and support for improved clinical care of older adults. Possible threats included perceived potential conflict with academic Divisions of Geriatric Medicine and Geriatric Psychiatry.

Discussion: A Family Medicine DCOE has general support from both internal and external stakeholders, with consensus that it could improve academic and clinical domains. The 
need to build strong relationships with Faculty of Medicine Divisions of Geriatric Medicine and Geriatric Psychiatry were highlighted. Models of successful DCOEs elsewhere in Canada will serve as potential guides.

Conclusion: A Family Medicine DCOE can serve as an academic home for family physicians interested in care of older adults. These findings could have applications for the development of similar divisions at other universities in Canada.

\section{Emergency Room Evaluation and Recommendations for Older Users of Emergency Departments: Results of ER2 Experimental Study}

C. Launay, A. Halme, M. Afilalo, O. Beauchet. Jewish General Hospital.

Background: The "Emergency Room Evaluation and Recommendations" (ER2) is a clinical tool designed to determine prognosis for short-term Emergency Department (ED) adverse events (i.e., long length of stay (LOS) in ED and hospital, hospital admission) during an index ER visit and to continue appropriate interventions. The study aims to examine whether ER2 recommendations may reduce the length of stay in ED and in hospital, and the hospital admission rate in older ED users on stretcher.

Methods: A total of 3,931 older ED users of the Jewish general Hospital (Montreal, Quebec, Canada) were recruited in this non-randomized, single arm, pre-post intervention open label trial. During the observational phase, ED staff was blind of ER2 score and patients received usual ED care, whereas during the interventional phase ED staff was informed about the ER2 score and related interventions as per ER2 recommendations were proposed. The length of stay in ED and in hospital, and the hospital admission rate were used as outcomes.

Results: The ER2 recommendations were associated with increased length of stay in $\mathrm{ED}(\beta=2.94$ with $\mathrm{P} \leq 0.001)$ but decreased length of stay in hospital $(\beta=-2.07$ with $\mathrm{P}=0.011)$. No effect was found on hospital admission ( $\beta=0.92$ with $\mathrm{P}=0.182$ ).

Discussion: The findings revealed that ER2 recommendations were significantly associated with increased length of stay in ED but decreased length of stay in hospital when the older ED users were admitted to hospital at the index ED visit. They also confirmed that ER2

Conclusion: ER2 recommendations had mixed effects. Shorter hospital stay has been reported for older ED users admitted at the ED index visit but increased ED stay and no effects on hospital admission were found.

\section{Frailty Screening in Older Adults in Primary Care: Is Annual Screening Necessary?}

L. Lee ${ }^{1}$, A. Jones ${ }^{2}$, L. M. Hillier ${ }^{3}$, A. Costa ${ }^{2}$, T. Patel ${ }^{4}$, J. Milligan ${ }^{2}$, R. Parikh ${ }^{5} .{ }^{1}$ Centre for Family Medicine
Family Health Team; McMaster University; ${ }^{2}$ McMaster University; ${ }^{3}$ Geriatric Education and Research in Aging Sciences (GERAS) Centre; ${ }^{4}$ University of Waterloo;

${ }^{5}$ Centre for Family Medicine Family Health Team.

Background: Centre for Family Medicine Case-Finding for Complex Chronic Conditions in Adults 75+ (C5-75) involves frailty screening in primary care using dual trait screening measures of gait speed and handgrip strength annually, with additional screening for associated complex conditions in those deemed frail. This study aimed to streamline C5-75 screening by identifying low-risk individuals who could be screened for frailty every two years rather than annually without substantively impacting yield.

Methods: This study examined a prospective cohort of patients in a family practice group in Kitchener, Ontario who completed at least two annual C5-75 screenings between April 2014 and December 2018. Grip strength and gait speed on initial assessment were categorized based on proximity to frailty thresholds and were used to predict frailty risk on the second assessment. Differences in risk were assessed using Fisher's exact test. Logistic regression models tested associations between age, patient activity level, falls history and risk of frailty on subsequent assessment, controlling for grip strength and gait speed.

Results: Analyses included 571 patients with two C5-75 assessments Frailty risk on the second assessment was significantly higher for patients who had gait speed or grip strength within $20 \%$ of the frailty threshold $(5.7 \%)$, compared to the other categories $(0.7 \%, 0.9 \%, 0 \% ; \mathrm{p}=0.002)$. Controlling for grip strength and gait speed, no other measures had significant associations with risk of frailty on the second assessment.

Discussion: Our results demonstrate that patients with whose gait speed and grip strength are both more than $20 \%$ over the frailty threshold at are low risk $(<1 \%)$ of transitioning to frailty by the next annual assessment.

Conclusion: Reducing screening frequently from annually to every two years may be appropriate for these patients.

\section{Frequency of Positive Screening for Comorbid Conditions in Older Adults Identified as Frail in Primary Care}

L. Lee ${ }^{1}$, A. Jones ${ }^{2}$, L. M. Hillier ${ }^{3}$, R. Parikh ${ }^{4}$, C. Lee ${ }^{4}$.

${ }^{1}$ Centre for Family Medicine Family Health Team,

McMaster University; ${ }^{2}$ McMaster University; ${ }^{3}$ Geriatric

Education and Research in Aging Sciences (GERAS)

Centre; ${ }^{4}$ Centre for Family Medicine Family Health Team.

Background: In the Centre for Family Medicine Case-Finding for Complex Chronic Conditions in Adults 75+ (C5-75), persons aged 75 years are systematically screened for frailty annually using gait speed and handgrip strength, and those identified as frail are screened for comorbid conditions 
associated with frailty. The purpose of this study was to identify the prevalence of these potential comorbid conditions.

Methods: This study examined a prospective cohort of family practice patients in Kitchener, Ontario who completed C5-75 screening 2013 to 2018. Level 1 of this 2-level algorithmic screening process screens for frailty based on gait speed and handgrip strength; those deemed frail in Level 1 move on to Level 2 multi-faceted screening for conditions associated with frailty using standardized tests for fracture risk, urinary incontinence, depression, anxiety, social isolation, caregiver burden if applicable, cognitive impairment, falls risk and risk of health destabilization. Frequencies were generated for all Level 2 assessment items where available.

Results: The cohort included 1,948 Level 1 C5-75 assessments and 195 Level 2 assessments, representing 159 unique patients. Positive screens were most frequent for urinary incontinence $(36.3 \% ; 70 / 193)$, falls $(27.8 \% ; 45 / 162)$, cognitive impairment $(25.6 \%$; 34/133), and depression $(20.9 \%$; 14/67). Less frequent positive screens occurred for high fracture risk $(19.5 \% ; 38 / 195)$, social isolation $(18.7 \%(35 / 187)$, caregiver burden $(16.7 \%$; $9 / 54)$, destabilization risk $(14.9 \% ; 17 / 114)$ and anxiety $(9.3 \% 10 / 98)$.

Discussion: Despite sample size limitations and recognizing positive screens do not confirm the condition, findings from this study suggest that frail older adults might have co-existing conditions that are unrecognized.

Conclusion: Identification of frailty and comorbid conditions provides an opportunity to better manage previously unidentified co-existing conditions that might affect, or be affected by, frailty.

\section{Who Is Being Assessed in Primary Care Memory Clinics? Insights From a Decade of Experience in One Multispecialty Interprofessional Team (MINT) Memory Clinic}

\section{Lee ${ }^{1}$, A. Jones ${ }^{2}$, L. M. Hillier ${ }^{3}$, R. Parikh ${ }^{4}$, C. Lee L $^{4}$. ${ }^{1}$ Centre for Family Medicine Family Health Team; McMaster University; ${ }^{2}$ McMaster University; ${ }^{3}$ Geriatric Education and Research in Aging Sciences (GERAS) Centre; ${ }^{4}$ Centre for Family Medicine Family Health Team.}

Background: Multispecialty Interprofessional Team (MINT) memory clinics provide comprehensive assessment and management of memory concerns within primary care. There are currently over 100 MINT clinics across Ontario. The Centre for Family Medicine (CFFM) Family Health Team MINT clinic, Kitchener, Ontario, has been serving patients since 2006. This study describes the profile of patients that have been referred to this clinic.

Methods: This study examined initial assessments of a cohort of patients assessed in CFFM memory clinic from June 2006 to May 2019. Data abstracted from patient records were: age, gender and assessment outcomes: Montreal Cognitive Assessment (MoCA) scores, Cornell depression scale scores, diagnoses, specialist referrals, and reporting of driving safety concerns to Ministry of Transportation. Frequencies were generated for all data.

Results: The cohort included initial memory clinic assessments for 752 patients. Average age was 75 years; $54 \%$ were female. The majority (67\%) had MoCA scores of 25 or less; $35 \%$ had scores of 20 or less. $33 \%$ screened positive for depression (Cornell scores $8>$ ). The majority $(57 \%$; $\mathrm{N}=422)$ were diagnosed with MCI $(36 \%)$ or dementia $(21 \%)$, mixed dementia being the most common $(65 \%$; $N=99 / 152)$. Other diagnoses included subjective cognitive decline (12\%) and depression (19\%, often concurrent); diagnoses affecting $<5 \%$ included delirium, medication-related memory effects, and post-concussive syndrome. $8 \%(\mathrm{~N}=59)$ were referred to a specialist (geriatrician, $4 \%$; neurologist, $3 \%$; psychiatrist, $1 \%$ ). Driving safety concerns were reported for $20 \%$ of patients.

Discussion: These findings highlight the profile of patients referred to MINT clinics.

Conclusion: Results are suggestive of the capacity of primary care memory clinics to assess and manage memory complaints related to a wide range of causes, reserving specialist referrals for $8 \%$ of cases.

\section{Using Point of Care Ultrasound (PoCUS) as a Marker for Muscle Mass and Muscle Strength in Older Adults}

\section{K. Madden, B. Feldman, S. Arishenkoff, G. Meneilly. University of British Columbia.}

Background: Sarcopenia is defined as the gradual ageassociated loss of both muscle quantity and quality in older adults, and severe sarcopenia is defined as sarcopenia that affects subject performance (gait speed). It is a devastating condition, predicting an increase in mortality, falls, fractures and hospitalizations. Current clinical criteria recommend the use of Dual X-ray Absorptiometry (DXA) to measure muscle mass, a test that cannot be performed at the bedside and is rarely used to screen for this condition. The objective of the current study was to examine Point-of care ultrasound (PoCUS) as a quick, non-invasive marker for both muscle mass and function.

Methods: 150 older adults (age $>=65$; mean age $80.0 \pm 0.5$ years, 66 women, 84 men) were recruited sequentially from 5 geriatric medicine clinics (Vancouver General Hospital). Each subject had appendicular skeletal muscle mass (ASM, by bioimpedance assay), grip strength, mid-arm biceps circumference (MABC), gait speed, and an ultrasonic measure of muscle quantity (MT, vastus medialis muscle thickness) measured. Our initial models contained age, sex, body mass index (BMI), and MT as predictor variables, and our outcome variables were ASM, grip strength, MABC and gait speed. 
Results: In our final parsimonious models, MT showed a strong significant correlation with all measures of muscle mass, including ASM (Standardized $\beta=0.204 \pm 0.058, \mathrm{R}^{2}=$ $0.58, \mathrm{p}<0.001$ ) and MABC (Standardized $\beta=0.141 \pm 0.067$, $\mathrm{R}^{2}=0.77, \mathrm{p}=0.038$ ). With respect to measures of muscle quality, there was a strong significant correlation with grip strength (Standardized $\beta=0.156 \pm 0.058, \mathrm{R}^{2}=0.51, \mathrm{p}=0.008$ ) but not with subject performance (gait speed).

Discussion: MT showed strong correlations with measures of muscle mass (ASM and MABC) and with muscle strength (grip strength).

Conclusion: Although further work is required, our results suggest that bedside ultrasonic measures of muscle thickness have potential as a rapid screening tool for sarcopenia.

\section{The Effect of a Multimodal Exercise Intervention Combined with Computerized Cognitive Training on Depressive Symptoms in Older Adults with Mild Cognitive Impairment: A Preliminary Report}

J. Mahon ${ }^{\text {, }}$ Q. Almeida ${ }^{2}$, L. Bherer ${ }^{3}$, A. Burhan ${ }^{4}$, T. LiuAmbrose $^{5}$, L. Middleton ${ }^{6}$, L. Nagamatsu ${ }^{1}$, M. Speechley ${ }^{7}$, M. Montero-Odasso ${ }^{8} .{ }^{1}$ Department of Kinesiology, Western University; ${ }^{2}$ Sun Life Financial Movement Disorders Centre, Wilfred Laurier University; ${ }^{3}$ Faculty of Medicine, University of Montreal; ${ }^{4}$ Parkwood Institute-Mental Health; ${ }^{5}$ Department of Physical Therapy, University of British Columbia; ${ }^{6}$ Department of Kinesiology, University of Waterloo; ${ }^{7}$ Department of Epidemiology and Biostatistics, Western University; ${ }^{8}$ Gait and Brain Lab, Parkwood Institute and Lawson Health Research Institute.

Background: Depressive symptoms (DS) are common in older adults with mild cognitive impairment (MCI) and confer a higher likelihood of progression to dementia. Two interventions that may reduce DS include physical exercise and computerized cognitive training (CCT). Currently, the impact of combining multimodal exercises with CCT programs on DS in older adults with MCI is unclear. Our objective was to evaluate whether a 5-month program combining exercise and CCT would result in a greater reduction of DS than either multimodal exercise or control groups alone, in older adults with MCI.

Methods: Data is obtained from the SYNERGIC trial, a RCT for this secondary analyses. 68 participants ages $\geq 60$ were randomized to one of three groups; Group 1: multimodal exercise and $\mathrm{CCT}(\mathrm{N}=27)$, Group 2: multimodal exercise and sham CCT $(\mathrm{N}=26)$, and Group 3: control group performing stretching exercises and sham CCT $(\mathrm{N}=15)$. All groups attended intervention sessions 3-times weekly. The Geriatric Depression Scale-30 (GDS-30) was used to measure DS at baseline and post-intervention. Two-way repeated measures ANOVA was used to evaluate the effect of different treatments on DS.
Results: Mean age was $74.30 \pm 5.88,52.9 \%$ female. Following intervention GDS-30 scores decreased by $20.62 \%$ and $4.08 \%$ for Groups 1 and 2 respectively, while increasing by $11.91 \%$ in the active control group $(\mathrm{p}=0.150)$. The interaction between time and treatment became significant $(\mathrm{p}=0.018)$ when using stratification analysis by GDS-30 $>9$.

Discussion: Our findings suggest this multimodal exercise and cognitive training intervention may be a viable option for reducing DS in older adults with MCI, especially in those with GDS-30>9.

Conclusion: A 5-month intervention combining multimodal exercise with cognitive training significantly reduced DS in older adults with MCI when compared with exercise training alone or control exercise.

\section{Overactive Bladder in Older People is Associated with Frailty But Not Comorbidity}

\section{R. Maradiya, A. Wagg, S. Rajabali. University of Alberta.}

Background: Overactive bladder (OAB) in older people has been associated with an increased likelihood of impaired performance on the timed up and go test (TUGT), a single facet of frailty. This study examined the relationship between a multidimensional frailty scale, the Edmonton Frail Scale (EFS), the degree of comorbidity and the presence of OAB in older patients.

Methods: Consecutive male and female patients ( $>65 \mathrm{y})$ attending a specialist continence clinic provided data for the EFS, TUGT, comorbid conditions and demographics. OAB was diagnosed according to the criteria of the International Continence Society. Data on comorbid conditions were used to calculate a Charlson Comorbidity Index (CCI). Frailty was defined as EFS $\geq 5$, impaired TUGT $>10$ s. Comparative analyses were then performed using a $\mathrm{OAB}$ and non-OAB (N-OAB) cohort. The N-OAB cohort comprised patients with other incontinence subtypes.

Results: There were $149 \mathrm{OAB}$ patients of mean (SD) age 80.1 (7.7) and $46 \mathrm{~N}-\mathrm{OAB}$ patients of mean (SD) age 78.9(7.4), $\mathrm{p}=\mathrm{NS}$. Patients were mostly female, OAB 113 (76.4\%), NOAB 35 (77.8\%). Mean TUGT was higher in OAB group, $16.9 \mathrm{v} 13.6 \mathrm{~s}, \mathrm{p}=0.017$. OAB group was more likely to be frail $71.2 \%$ v $50.0 \%, p=0.021$. Mean CCI (1.6) was no different between groups

Discussion: OAB, particularly urinary urgency is associated with frailty in older people using EFS. Urgency particularly, is related to increased risk of falls, deconditioning and cerebral disease in older people. To what extent successful treatment affects frailty, if at all remains to be seen

Conclusion: $\mathrm{OAB}$ is yet another facet of the frailty syndrome in older people. 


\section{A Performance Measure Framework for Evaluating Community Based HealthCare for Older Adults}

D. McCaughey, G. McGhan, N. Ludlow, W. Ghali. University of Calgary.

Background: The increasing proportion of older Canadians living in rural and remote areas requires rethinking how healthcare services are provided. Community Based Health Care (CBHC) is an integrated health system structured around individuals and communities to enhance how people are connected to healthcare and services. CBHC has the potential to bridge the gap for better access, integration, and continuity of care that is person-centred and increases improved care options closer to home. When implemented, it has the potential to improve the value of care, the experience of care, and health outcomes for the population including older adults. A framework is needed to measure the expected outcomes that result from CBHC. As such, working in collaboration with a provincial health ministry, we co-developed an evaluation framework for CBHC.

Methods: Mixed methods were used to identify possible indicators and a modified Delphi to rank and group indicators within the health system.

Results: A total of 26 indicator sets transcended programs within the provincial healthcare system. At the highest tier (tier one: system outcome measures), identified indicator sets include quality of life, avoidable hospitalization, and access to integrated primary care. Tier two (strategic measures), indicator sets include effective palliative care, geographical primary care coverage, and continuing care capacity. Finally, tier three measures (tactical/transactional), include indicator sets of team-based care, community paramedicine capacity, and access to home care services.

Discussion: Indicators were found to be integrated across tiers and serve to link care services at all levels thereby creating a system level framework to organize healthcare for Canadians of all ages, particularly older adults.

Conclusion: If implemented and evaluated systematically, $\mathrm{CBHC}$ provides an opportunity to address inequitable healthcare service delivery, positively impacting our rural and/or remote ageing population.

\section{A Tailored Community Program for the People Living with Dementia and Their Family Caregivers}

G. McGhan ${ }^{1}$, K. Shapkin ${ }^{1}$, J. P. Cyr ${ }^{2} .{ }^{1}$ University of Calgary; ${ }^{2}$ The Glencoe Club.

Background: Tailored care strategies have potential to address declines in physical and cognitive functioning for people living with dementia (PLWD) while making a positive difference in their daily lives; however, these services are not commonplace. The purpose of this study is to examine a tailored community dementia program (TCDP) prescribed for individual abilities of PLWD and measure the impact on the caregiving dyad.

Methods: A mixed method design was used for a 12-week TCDP with 13 dyads living in the community. PLWD and caregivers completed assessments at baseline, 6, 12 and 18 weeks. Daily activity was measured in PLWD with no decline observed during the assessment period. Measurements for family caregivers included caregiver strain, satisfaction and assessment of the PLWD's abilities.

Results: Highlighted themes included the importance of valuable socialization and comradery as well as the engagement the TCDP fostered. Many of the participants emphasized the social isolation they feel as a family caregiver or PLWD and that this program helped them build a social group in their community of people experiencing similar challenges. One of the PLWD stated "The friendships I made during the program were very important...the comradery was very meaningful", while one of the family caregivers said "I feel he has really benefited from this program. It keeps him engaged with people and I do think he is more fit."

Discussion: Although quantitative findings were not significant, qualitative findings from focus groups and caregiver journals highlighted several important themes. There was also general consensus on the benefits of tailoring activities to the ability of PLWD to promote participation.

Conclusion: Overall, the TCPD shows promise as a meaningful intervention, with the ability to be tailored to the needs and resources of the caregiving dyad.

\section{Clinical and Cost Effectiveness of a Comprehensive Geriatric Assessment and Management for Canadian Elders with Cancer: The 5C Study-Near Final Recruitment and Implementation Results}

B. McLean ${ }^{1}$, S. Alibhai ${ }^{2}$, F. Strohschein ${ }^{3}$, N. Alqurini ${ }^{4}$, A. Li ${ }^{5}$, C. Mariano ${ }^{6}$, R. Mehta ${ }^{7}$, J. Monette ${ }^{3}$, D. WanChow-Wah ${ }^{3}$, M. Puts ${ }^{8} .{ }^{1}$ Michael G. DeGroote School of Medicine, McMaster University; ${ }^{2}$ Department of Medicine, University Health Network and Institute of Health Policy, Management and Evaluation, University of Toronto; ${ }^{3}$ Division of Geriatric Medicine, Jewish General Hospital; ${ }^{4}$ Department of Medicine, University Health Network; ${ }^{5}$ Geriatric Medicine, Royal Columbian Hospital; ${ }^{6}$ Medical Oncology, Royal Columbian Hospital; ${ }^{7}$ Division of Geriatric Medicine, Sunnybrook Health Sciences Centre; ${ }^{8}$ Lawrence $S$. Bloomberg Faculty of Nursing, University of Toronto.

Background: Geriatric assessment and management is recommended for older adults with cancer considering chemotherapy, but no definitive randomized controlled trial (RCT) has been completed in Canada. Our objective is to report on 
the successes and challenges faced in implementation of the multicentre $5 \mathrm{C}$ trial.

Methods: Patients aged 70+ with cancer and referred for chemotherapy are recruited and randomized (single blind, outcome assessor). Patients randomized to intervention receive a comprehensive geriatric assessment and management for 6 months. Outcomes such as quality of life and cost effectiveness are measured via monthly validated questionnaires and health care cost diaries.

Results: Recruitment started April 2018 in $2 / 8$ centres. All sites are currently recruiting. Three hundred twenty participants have been recruited ( $91 \%$ of target) and 63 (18\% of target) have completed the study. Approximately $10 \%$ of follow-ups are missed each month due to participant illness, difficulty contacting the participant, or other reasons. Recruitment success ranges from $20 \%$ to $76 \%$. Larger, more research-intensive hospitals are recruiting more patients in total $(70 \%$ of total participants are from one centre). However, recruitment success does not seem to correlate with hospital size. Participant dropout for reasons other than death has been very low $(n=16,5 \%)$.

Discussion: The implementation of this multicentre RCT across 3 provinces in 2 languages has taken at least $50 \%$ longer than projected. Recruitment was delayed due to the differing timelines in opening recruitment at the different hospitals and the lower than anticipated recruitment success at several centres.

Conclusion: Site-specific adaptations, regular communication among teams, minor protocol modifications and a personcentered approach in participant interactions have promoted recruitment and retention of participants. Recruitment is expected to be completed within the next few months and data collection will finish one year later.

\section{A Prospective Observational Case Control Study of Possible Adverse Effects of Antidepressants in Patients with Cognitive Impairment}

\author{
S. McMullin ${ }^{1}$, E. Levinoff ${ }^{2}$, H. Hagh-Doust ${ }^{1} .{ }^{1}$ McGill \\ University; ${ }^{2}$ Jewish General Hospital.
}

Background: Depression is a prevalent symptom of dementia. However, it is unclear whether treatment with antidepressants shows symptom improvement. Antidepressant side effects are studied in the elderly, but there is a paucity of data on which to base medical decisions. Furthermore, polypharmacy can lead to adverse outcomes, including a higher risk of falls. In this study, we used the Cornell Scale for Depression in Dementia (CSDD) to assess symptom control in hospitalized patients taking antidepressants at the time of admission and to explore the relationship between symptom control and risk of falls.

Methods: A prospective observational case control study was performed. The CSDD was administered to the caregivers of 44 participants. Various demographic data, including falls, number of medical comorbidities, number of medications on admission, benzodiazepine use, antipsychotic use, and MMSE scores were collected. Chi-square, t-tests and logistic regression where used in the statistical analysis.

Results: $61.4 \%$ of the 44 patients where female and the mean age was 85.0 years. The admitting diagnosis was falls in $36.4 \%$ of cases, and $61.4 \%$ of patients had sustained a fall in the past year. Using a cut-off score of 8 on the CSDD, there was a statistically significant association between a CSDD > 8 and a decreased risk of falls in the past year. We could not prove a statistically significant association between a higher CSDD score and hospital admission for falls.

Discussion: In patients taking antidepressants, a CSDD score above 8 is associated with a lower risk of falls in the past year. Apathy and somatic symptoms could represent contributing factors to this.

Conclusion: We suggest that physicians working with older adults routinely screen for falls in all patients.

\section{The Effect of a Geriatric Consultation Team on Indicators of Care in Clinical Teaching Units Following Implementation of the Age-Friendly Hospital Principles}

J. A. Morais ${ }^{1}$, H. T. Leite ${ }^{2}$, L. A. Antunes ${ }^{3}$, G. H. Boutros ${ }^{4}$, A. C. Manhaes ${ }^{5} .{ }^{1}$ Division of Geriatric Medicine, McGill University Health Care; ${ }^{2}$ Hospital Adventista Silvestre and Instituto Nacional de Cardiologia, Rio de Janeiro, Brazil; ${ }^{3}$ Hospital Adventista Silvestre, Rio de Janeiro, Brazil; ${ }^{4}$ McGill University Health Centre; ${ }^{5}$ Universidade de Rio de Janeiro, Rio de Janeiro, Brazil.

Background: The current model of hospital care often presents itself to older patients with characteristics that are hostile. The Age-friendly Hospital (AFH) concept in an approach to the older inpatient at risk of functional decline, but the evidences of a geriatric consultation team in optimizing its principles are lacking. Our objective was to demonstrate the effect of a geriatric consultation team on clinical administrative indicators of care in the Clinical Teaching Units (CTUs) of the Hospital Adventista Silvestre, Rio de Janeiro, Brazil who have adopted the AFH principles

Methods: Following at least 3 months of implementation of the AFH principles in CTUs, two periods of the same 6 months of the year (January to June 2015 and 2016) were compared, corresponding to a Pre and Post geriatric consultation team intervention on vulnerable older inpatients $>60 \mathrm{y}$ based on PRISMA-7 instrument. Clinical administrative data were collected and analyzed.

Results: In Pre vs. Post periods. There were 641 vs. 743 admissions to CTUs. Average length of inpatient stay $( \pm \mathrm{SD})$ : $8.7 \pm 2.7$ vs. $5.4 \pm 1.7 \mathrm{~d}(\mathrm{p}=0.03)$, ombudsman complaints/ month (n): $44.2 \pm 16.0$ vs. $13.5 \pm 5.5(\mathrm{p}=0.03)$, total hospital costs/patient: $3018 \pm 1363$ vs. $2319 \pm 1114$ USD ( $p=0.20)$, 
30-d readmission/month (\%): $9.2 \pm 6.8$ vs. $4.6 \pm 1.1(\mathrm{p}=0.13)$, homecare services/month (n): $2.5 \pm 2.4$ vs. $38.3 \pm 15.4$ ( $\mathrm{p}=0.01$ ).

Discussion: The presence of the geriatric team was successful in reducing length of stay and complaints to the ombudsman without increasing overall hospital costs. The $30-\mathrm{d} \%$ readmission rate despite a trend did not reach significance while referral to homecare support was increased.

Conclusion: The presence of a geriatric consultation team is of added benefit to increase the efficiency of the AFH for vulnerable older inpatients without increasing hospital costs and includes more referrals to homecare services.

\section{Developing the Driving Cessation in Dementia Toolkit (DCDT): A Knowledge-to-Action Process}

G. Naglie ${ }^{1}$, E. Stasiulis ${ }^{1}$, S. Sanford ${ }^{1}$, M. Bédard ${ }^{2}$, P. Belchior ${ }^{3}$, A. Byzewski ${ }^{4}$, A. Crizzle ${ }^{5}$, I. Gélinas ${ }^{3}$, S. Marshall ${ }^{4}$, B. Mazer ${ }^{3}$, F. Molnar ${ }^{4}$, P. Moorhouse ${ }^{6}$, A. Myers ${ }^{7}$, J. Polgar ${ }^{8}$, M. Porter ${ }^{9}$, H. Tuokko ${ }^{10}$, B. $V_{\text {Vkljan }}{ }^{11}$, S. Yamin ${ }^{12}$, M. Rapoport on behalf of the CCNA Driving Cessation Team Co-Investigators and CCNA study group. ${ }^{1}$ Baycrest Health Sciences; ${ }^{2}$ Lakehead University; ${ }^{3}$ Mc Gill University; ${ }^{4}$ University of Ottawa; ${ }^{5}$ University of Saskatchewan; ${ }^{6}$ Dalhousie University; ${ }^{7}$ University of Waterloo; ${ }^{8}$ Western University; ${ }^{9}$ University of Manitoba; ${ }^{10}$ University of Victoria; ${ }^{11}$ McMaster University; ${ }^{12}$ St. Paul University, Sunnybrook Health Sciences.

Background: Despite the well-known challenges that driving cessation poses for people with dementia (PWD) and their family caregivers (FC), there are few evidence-based interventions and resources to support them. To address this gap we used a Knowledge-to-Action approach to develop a Driving Cessation Intervention Framework (DCIF) and Driving Cessation in Dementia Toolkit (DCDT).

Methods: In the Knowledge Creation phase of the KTA we conducted a series of knowledge synthesis activities including systematic reviews and a meta-synthesis. Knowledge inquiry activities involved qualitative studies with PWD, FCs and healthcare/service providers (HCSP). The second part of this phase, comprised a process of collecting and reviewing available tools and resources, curated according to the DCIF. In the Action Cycle phase we adapted early versions of the DCDT according to feedback from Alzheimer Society staff and an Advisory Committee of PWD and FCs.

Results: Knowledge Creation activities resulted in the production of the DCIF and the DCDT. The DCIF identified key elements of the decision-making and transition process to non-driving (e.g. awareness, communication, grief and loss), which along with Action Cycle activities informed our development and refinement of the DCDT-a web-based collection of curated tools and resources with guided access to specific content and unique portals for each target audience (i.e. PWD, FCs, HCSPs).
Discussion: In using a KTA framework we employed a systematic approach to the creation, application and promotion of driving cessation knowledge. The involvement of knowledge users in an integrated and iterative manner throughout the development process was essential to ensuring the relevance and uptake of the DCDT.

Conclusion: The KTA framework played an integral role in developing a toolkit with the potential to enhance the safety and quality of life for PWD and FCs.

\section{Current use of Beers ${ }^{\circledR}$ Criteria medications and Pharmacogenomic Interpretation}

N. Nemet, C. Sadowski, P. Mayo. University of Alberta.

Background: Safe medication use by older adults requires more selective prescribing and monitoring parameters; the Beers ${ }^{\circledR}$ Criteria is a recognized tool designed to aid clinicians in this area. Another approach is using pharmacogenomics (PGx), the study of how individuals differ in their response to drugs. The primary objective of this study was to see if a high proportion of Beers Criteria medications are being used within a specialized geriatric inpatient setting. Secondly, to determine if there is an overlap with PGx and drug safety outcomes in these older adults.

Methods: Inpatient data regarding age and medications for older adults in an Edmonton rehabilitation hospital was obtained. A PGx reference table was created using international guidelines, then cross-referenced with the Beers ${ }^{\circledR}$ Criteria 2019, specifically looking at medications to be avoided or with cautioned use in older adults. Information outlined was the medication, the genetic variant involved, the predicted effect and comments from the Beers ${ }^{\circledR}$ Criteria.

Results: Data was obtained for a total of 142 patients, mean age 79 (8). They were on a mean of 16 medications. 114 of these patients were using Beers Criteria medications, with the most prevalent drug classes included psychotropic medications $(n=67)$, PPIs $(n=60)$, and diuretics $(n=47)$. A total of 42 PGx-testable medications were identified, 37 of those present in the Beers ${ }^{\circledR}$ Criteria, associated with $n=88$ patients using a PGx testable medication.

Discussion: Many older adults in a geriatric-focused rehabilitation setting are using high risk medications. There is significant overlap in recommendations from PGx testable medications and Beers ${ }^{\circledR}$ Criteria for drug safety outcomes.

Conclusion: Drug safety may be improved in the future if PGx testing were implemented prior to using these medications.

\section{Audit of Geriatric Burn Patients' Chart to Improve Delirium Management}

E. Norris-Roozmon ${ }^{\text {, S. S. Danho }}{ }^{2}$, R. Mehta ${ }^{3}$, M. Norris ${ }^{3}$, D. Gandell ${ }^{3} .{ }^{1}$ Queen's University; ${ }^{2}$ University of Toronto, Sunnybrook Health Sciences Centre; ${ }^{3}$ Sunnybrook Hospital Health Sciences Centre. 
Background: Canada's aging population demonstrates increased vulnerability to burn injuries compared to younger adults as a result of increased co-morbidity and reduced physiological reserves. The geriatric burn population experiences a significantly higher burden of delirium, which is a syndrome less recognized among the burn unit health care team.

Methods: We aimed to audit a cohort of older-adults $(\geq 65)$ admitted to the Ross Tilley Burn Centre (RTBC) at the Sunnybrook Health Sciences Centre between 2017-2019. Baseline and discharge demographics, Charlson Comorbidity Index (CCI), Rockwood Clinical Frailty Scale (RCFS), Burn Surface Area (BSA), psychotropic medications and discharge disposition were abstracted. The primary aim was to evaluate the incidence and prevalence of delirium in older adults admitted to a specialized burn unit. Secondary aims were to characterize the sample and to evaluate adherence to geriatric consultation team recommendations.

Results: 43 patient (28 Male, 15 Female) charts were abstracted. Average age was 76 years, with a mean BSA of $11.8 \%$. Mean RCFS score pre-admission was 3.8 and 4.9 after admission, with an average $\mathrm{CCI}$ of 5.1. Geriatric consultation, identified 24/43 (55.8\%) patients with acute confusion. 91.6\% of patients identified with acute confusion were no longer delirious upon discharge. Pharmacologic recommendations were routinely followed. However, non-pharmacological delirium prevention recommendations were infrequently implemented.

Discussion: Increased attention to medication appropriateness and daily patient interaction to promote cognition and function may be the underlying mechanism for accelerated delirium resolution in older adult burn victims although the CAM screening tool was not standard practice.

Conclusion: A retrospective chart review of burn patients aged $\geq 65$ years provides the first step in a quality improvement initiative to improve delirium outcome on a burn unit. Allied health engagement and CAM_ICU teaching are identified as targets for improvement.

\section{Collaboration Leading to Expedited Admission and Return to Long Term Care (CLEAR LTC)}

W. Ocampo ${ }^{1}$, A. Herring ${ }^{2}$, V. Ewa ${ }^{2}$, P. Jamieson ${ }^{3}, T$. Chan $^{3}$, M. J. Shankel ${ }^{2}$, J. Holroyd-Leduc ${ }^{3}$, M. Grinman ${ }^{3}$, N. Virk ${ }^{4}$, M. Brophy ${ }^{2}$, A. Heinemeyer ${ }^{2} .{ }^{1}$ W21C Research and Innovation Centre, University of Calgary; ${ }^{2}$ Alberta Health Services, ${ }^{3}$ Alberta Health Services and University of Calgary; ${ }^{4}$ The Brenda Strafford Foundation Ltd.

Background: Minimizing exposure of frail older adults to hospitalization is crucial in reducing the risk of iatrogenic complications including, adverse events secondary to invasive investigation and treatments, delirium, functional decline, responsive behaviours, and falls. The CLEAR-LTC project aims to expedite discharges back to long term care (LTC) homes, decrease admission-related adverse events for LTC residents, while aiming to optimize acute care resources for this population.

Methods: All patients admitted from five LTC sites to a Calgary tertiary hospital were identified upon presentation to acute care. The intervention involved engaging and facilitating the admitting hospitalist physician to phone these patients' LTC physicians' within 48 hours of hospital admission. Using a pre-post design, a 7-month interim analysis was done on the primary outcome of acute hospital length of stay (ALOS), as well as 7-day and 30-day hospital readmission rates. Qualitative data was obtained through surveys and interviews with patients, families and physicians.

Results: The mean ALOS and standard deviation for patients admitted pre-intervention was $11.9+14.4$ days compared to $7.2+5.7$ days for post-intervention ( $p$-value $=0.032$ ). There was no significant difference in readmissions. Twenty-three out of 43 physicians reported having a conversation, with half of them citing the call as being valuable.

Discussion: The significant reduction in ALOS indicates that a phone conversation between the hospitalist and LTC physician can facilitate earlier discharge back to LTC homes, where ongoing acute care can still be appropriately provided for lower acuity issues. The conversation provides an opportunity for physicians to discuss rationale for admission and patients' care needs during and after hospitalization.

Conclusion: A phone conversation at the time of hospital admission between the hospitalist and LTC physician can expedite patients' discharge back to LTC but does not impact readmission rates.

\section{Skin Tear Management: Collaborative Physician- Nurse Education Project}

C. Ott, T. Yogaparan, A. Vecchio, T. Dayal, D. R. Gomez, C. Brinton, A. Berall. Baycrest Hospital.

Background: Skin tears are common in older adults and often neglected in physician and nursing education. We wanted to create and implement a common educational program for physicians and nurses on skin tears. Having two parallel systems of education programing takes resources and time that could be spent elsewhere. Our aim is to evaluate the impact of skin tear education quality improvement (QI) initiative on physician and nurse knowledge and practices.

Methods: A literature search was conducted and educational module was developed on skin tears. Plan was to present it to physicians and nurses and evaluate post education content knowledge, learner satisfaction and confidence level. First physician education component was completed during family practice rounds. Program was presented to 9 physicians at Baycrest hospital. The next day a survey questionnaire was emailed.

Results: 7 of 9 physicians responded. Questions were asked about satisfaction, knowledge, confidence building, 
helpfulness in learning, and helpfulness in coordinating skin tear management. For satisfaction, 2 reported satisfied and 5 were very satisfied. For increasing knowledge, 2 reported a moderate amount of increase, 3 reported their knowledge increased a considerable amount and 2 reported their knowledge increased a great deal. Confidence increased moderately for 3 , considerable amount for 3 and a great deal for 1 . For identifying and classifying skin tears, 5 found it very helpful and 2 found it extremely helpful. Helpfulness in coordinating skin tear management 4 found it moderately helpful and 3 found it very helpful. More results to follow: content answers and questionnaires following nursing education.

Discussion: There was overwhelming enthusiasm and support for skin tear education by the physicians.

Conclusion: Next step will be teaching the same program to the nursing staff followed by a questionnaire. We will be developing an online module as well.

\section{FitJoints: Getting Fit for Hip and Knee Replacement: A Feasibility Randomized Controlled Trial}

A. Papaioannou, A. Negm, G. Ioannidis, J. Lee, S. Marr, M. Winemaker, D. Petruccelli, C. Kennedy, J. D. Adachi, A. Lau, S. Atkinson, J. DeBeer, V. Avram, B. Deheshi, D. Williams, B. Lumb, D. Armstrong, A. Panju, J. Richardson, L. Thabane. McMaster University.

Background: Pre-operative frailty has a high risk for post-operative complications, increased length of stay, and discharge to long-term care. Multi-modal interventions reduce frailty in patients under geriatric care. The purpose of the FitJoints RCT is to examine the feasibility of a pre-operative multi-modal intervention in pre-frail/frail older adults undergoing elective hip or knee replacement.

Methods: 69 participants with a mean age (standard deviation (SD)) of 73.9 (7.5) years were recruited and randomized to two groups. The control group received usual care and the intervention group received a tailored exercise program, protein and vitamin D supplements, and a medication review. Feasibility outcomes were recruitment rate, intervention completion, and adherence. Clinical outcomes were frailty (Fried Phenotype), physical function (Short Performance Physical Battery (SPPB), Oxford Hip (OHS) and Knee Scores (OKS)), and quality of life (EuroQol Group 5-Dimension 3-Level (EQ-5D-3L)). Outcome measures were collected at baseline, 1-week pre-operative, 6-weeks post-operative, and 6-months post-operative.

Results: A total of 56/69 participants completed the study, $83 \%$ of which completed the intervention. Screening and recruitment rates were $19.2 \%$ and $35 \%$ respectively. Adherence to exercise sessions, protein supplement, vitamin D supplement, and medication review were $68.4 \%, 87.5 \%, 85.7 \%$, and $100 \%$, respectively. Percent of participants pre-frail and frail were $50.7 \%$ and $36.2 \%$, respectively. Mean score (SD) of the SPPB test was 6.7 (2.3), OHS was 40.5 (7.3), OKS was 35.5 (7.2), and EQ-5D-3L was 70 (16.3).

Discussion: Strengths of the study include the use of a multimodal intervention and engagement of key stakeholders in the development and implementation process. Limitations include non-generalizability given that recruitment took place at one hospital and heterogeneity in intervention durations due to variability in surgical wait-times.

Conclusion: The FitJoints methodology is feasible to conduct a large multisite trial.

\section{Co-Designing Caregiver-Centered Care Health Workforce Training: Making the Leap to Support Family Caregivers of People Living with Dementia}

J. Parmar ${ }^{1}$, L. Poole 2 , S. Anderson ${ }^{1}$, W. Duggleby ${ }^{3}$, J. Holroyd-Leduc ${ }^{4}$, S. Brémault-Phillips ${ }^{5}$, L. Charles ${ }^{6}$, A. Haq ${ }^{7} .{ }^{1}$ Department of Family Medicine, University of Alberta; ${ }^{2}$ Dementia Advocacy Canada, Calgary; ${ }^{3}$ Faculty of Nursing, University of Alberta; ${ }^{4}$ Division of Geriatric Medicine, Departments of Medicine and CHS, University of Calgary; ${ }^{5}$ Department of Occupational Therapy, Faculty of Rehabilitation Medicine, University of Alberta; ${ }^{6}$ Department of Family Medicine Faculty of Medicine and Dentistry, University of Alberta; ${ }^{7}$ Covenant Health Network of Excellence in Seniors' Health and Wellness.

Background: Health workforce training is an innovative approach to addressing inconsistent system of supports for family caregivers [FCGs]. Currently FCGs are marginalized by the health system, yet FCGs are the backbone of the health system, providing over $80 \%$ of the care for people with dementia, chronic illness and impairments. This backbone desperately needs support. Training programs that prepare health providers to effectively identify, engage, assess, and support FCGs will be essential to sustaining the family caregiving that reduces health system costs while maintaining the health and well-being of the FCGs.

Purpose: We will describe the Health Workforce CaregiverCentered Care Education co-design process and present the educational modalities created in alignment with validated Caregiver-Centered Care Competencies.

Methods: We engaged over 100 multi-level, interdisciplinary stakeholders including FCGs to co-design Caregiver-Centered Care Education for the Health Workforce underpinned by collaborative working relationship between FCGs and healthcare providers.

Results: Multi-level, interdisciplinary stakeholders worked together to outline the competency-based-curriculum for caregiver-centered care for the health workforce and develop a facilitator guide and competency-based educational modules.

Discussion: The hallmark of effective education is content relevant to learners' needs and contexts, thus teaching and 
learning resources include competency-aligned educational modules, multimedia resources, and facilitators guide that are designed flexibly, to be tailored to setting and learners. We will pilot tested the Caregiver-Centered Care Education, for acceptability and effectiveness, in five care settings: primary, acute, homecare, supportive-living, and long-term care.

Conclusion: Our education will empower healthcare providers in supporting family caregivers in their caregiving role, decisions about services, care management, and advocacy. Health workforce support will enable FCGs to continue to play a vital role as key partners in the health system.

\section{Validating the Health Workforce Core Competencies to Support Family Caregivers of Older Adults}

J. Parmarl , L. Poole 2 , S. Anderson', W. Duggleby', J. Holroyd-Leduc ${ }^{5}$, S. Brémault-Phillips ${ }^{6}$, C. Pollard ${ }^{7}$, W. Jansen ${ }^{8}$, A. Haq ${ }^{9}$. 'Department of Family Medicine, University of Alberta; ${ }^{2}$ Dementia Advocacy Canada, Calgary; ${ }^{3}$ Department of Family Medicine, University of Alberta; ${ }^{4}$ Faculty of Nursing, University of Alberta; ${ }^{5}$ Division of Geriatric Medicine, Departments of Medicine and CHS, University of Calgary; ${ }^{6}$ Department of Occupational Therapy, Faculty of Rehabilitation Medicine, University of Alberta; ${ }^{7}$ Bachelor of Science in Nursing, McEwan University; ${ }^{8}$ Athabasca University; ${ }^{9}$ Covenant Health Network of Excellence in Seniors' Health and Wellness.

Background: Family caregivers [FCGs] provide over $80 \%$ of the care for adults with chronic progressive illnesses and age-related needs. Despite evidence of their contributions and the consequences caregiving, FCGs support has not been a health system priority. Over 300 Alberta multi-level interdisciplinary stakeholders recommended education of the healthcare workforce on engaging and supporting FCGs. Our objective was to validate the core competencies to guide health workforce education design and the measurement of the education impacts.

Methods: We used a Modified Delphi approach. In the first 2 rounds, multi-level, interdisciplinary participants rated the indicators in terms of importance and relevance. In the 3rd round consensus meeting, large and small group sessions were utilized to further finalize the competency indicators.

Results: Expert panel of 42 international, national, and provincial stakeholders participated. Thirty-four experts (81\%) participated in the round $1,36(85.7 \%)$ in round 2 , and 42 people (100\%) in round 3 . There was stable consensus across all three rounds, $96.07 \%$ of participants rated the indicators as essential or important (Round 1, 95.81\%; Round 2\%, 94.15; Round 3, 98.23\%). Six competency domains, including indicators, were validated as follows: Recognizing the Caregiver Role; Communicating with FCGs; Partnering with FCGs; Fostering Resilience in FCGs; Navigating the Health and Social Systems; Enhancing the Culture and Context of Care.

Discussion: Caregiver research has primarily focused on educating caregivers to provide care. We believe that our stakeholder engagement has advanced a population health approach in which family caregivers can be supported throughout the care trajectory, by a health workforce trained to support FCGs.

Conclusion: These competencies are shaping the design of educational curricula and interdisciplinary training programs aimed at supporting the health workforce to provide caregivercentered care.

\section{Physician Feedback on the Use of a Computerized Decision Support Tool at the Long-Term Care Medication Review}

\author{
G. Perri ${ }^{1}$, E. McDonald ${ }^{2}$, A. Berall ${ }^{1}$, C. Brinton ${ }^{1}$, \\ A. T. Santiago ${ }^{I}$, A. Moser ${ }^{l} .{ }^{1}$ Baycrest $;{ }^{2}$ McGill University \\ Health Centre.
}

Background: Polypharmacy is common in older adults, with two thirds of Canadian seniors prescribed five or more medication classes and nearly a third prescribed ten or more. While polypharmacy may be appropriate, it can also be associated with potentially inappropriate prescribing and an increased risk of adverse drug events. Deprescribing interventions, such as medication reviews and computerized decision support (CDS), can reduce inappropriate polypharmacy and may improve clinically significant health outcomes. The purpose of this study was to implement a CDS tool to facilitate deprescribing at the long-term care (LTC) quarterly medication review $(\mathrm{Q} 3 \mathrm{M})$ and obtain physician feedback.

Methods: Medical history and medications for 55 LTC residents were entered into the tool, which identified deprescribing opportunities based on polypharmacy guidelines. The clinical team reviewed the opportunities at the Q3M and made medication changes as appropriate. Physician feedback was collected on using the tool.

Results: Deprescribing opportunities were identified for 53 residents (96.4\%) and implemented for 18 (32.7\%). Physicians found the tool helpful in identifying some of the deprescribing opportunities and felt the tool would be useful for physicians without access to a pharmacist at the Q3M. Physicians felt it would be better to use the tool to prepare for the Q3M, allowing more time for interdisciplinary Discussion: about clinical application of guidelines during the Q3M. Quality improvement opportunities identified included incorporating medication administration information into the tool (e.g. crushed versus whole).

Discussion: CDS was successfully integrated with the LTC Q3M and nearly a third of deprescribing opportunities were implemented. The tool may be useful in educational contexts and earlier in the deprescribing decision-making process. 
Conclusion: Further research is required to optimize CDS for deprescribing within LTC and determine its impact on polypharmacy and clinical outcomes.

\section{Cholinesterase Inhibitor Use in Patients with Severe Dementia Admitted to a Palliative Care Unit}

G-A. Perri ${ }^{1}$, J. Wilson ${ }^{1}$, A. Kirstein ${ }^{1}$, S. Gardner ${ }^{l}$, A. Berall ${ }^{1}$, H. Khosravani ${ }^{2} .{ }^{1}$ Baycrest Health Sciences Center; ${ }^{2}$ Sunnybrook Health Sciences Centre.

Background: Dementia is more prevalent as an illness managed by palliative care. Current guidelines suggest that patients with severe dementia on cholinesterase inhibitors (CHEI) discontinue their CHEI by taper. This study looked at the prevalence of patients admitted to a palliative care unit (PCU) with severe dementia on a CHEI and whether CHEI deprescribing guidelines were followed.

Methods: This study was a descriptive retrospective chart review of patients admitted to a PCU with severe dementia admitted on a CHEI from January 2015 to June 2019. Data was collected to determine whether and how patients were titrated off their CHEI.

Results: A total of 36 patients were admitted to the PCU with severe dementia on a CHEI (2.4\% prevalence). The average age was 85.5 years with $56 \%$ of participants being female. The median length of stay was 21 days. For 31 of these patients, their CHEI was discontinued, only 9 of which had a taper. Of the 22 patients who discontinued their CHEI suddenly, 10 patients had an order to discontinue their CHEI in the last 2 days before their date of death.

Discussion: This study suggests that although patients admitted to a PCU with severe dementia have their CHEI discontinued, the discontinuation was done without a taper. More so, it appears that many patients had their CHEI continued through the active stage of dying, which may relate to family wishes or a sudden decline to functional status.

Conclusion: Considering the average length of stay for these patients on the PCU, it's surprising that CHEIs were not tapered earlier in the illness trajectory. Future work should explore reasons why PCU physicians are mostly late to taper CHEIs for patients admitted with severe dementia.

\section{Dementia and Stroke Comorbidity Among Canadians Aged 65 and Over}

\section{S. Plebon-Huff, L. Shaver, C. Robitaille, C. Pelletier. Public} Health Agency of Canada.

Background: As the population ages, more Canadians are living with multiple chronic conditions. This study describes prevalence and mortality (all-cause) patterns of comorbid dementia and stroke among Canadians aged 65+.
Methods: Prevalence and all-cause mortality rates and rate ratios for comorbid dementia and stroke among Canadians aged 65+ were calculated using data from the Canadian Chronic Disease Surveillance System (CCDSS). The CCDSS identifies chronic disease cases in provincial/territorial administrative health databases (hospitalization, physician billing, prescription drug databases) linked to provincial/territorial health insurance registries, using a unique personal identifier. Results have been disaggregated by sex and 5-year age groups.

Results: In 2016-17: About 1.8\% (over 110,000) of Canadians aged $65+$ had comorbid dementia and stroke, while $5.1 \%$ were living with dementia/without a stroke, and $7.8 \%$ had had a stroke/without dementia. The crude prevalence of this comorbidity was higher in women and increased abruptly with age, from $0.2 \%$ in those aged $65-69$ to $8.9 \%$ in those aged $90+$. The all-cause mortality rate among Canadians aged 65+ with the comorbidity, with dementia/without a stroke, and who had a stroke/without dementia was respectively $6.9,4.5$ and 2.6 times greater, compared to those without this comorbidity.

Discussion: Enhancing the evidence on dementia comorbidities supports the surveillance and data pillar of the National Dementia Strategy. Future surveillance work may explore dementia comorbidity trends over time and examine the sequencing of the comorbidities.

Conclusion: Over 110,000 Canadians aged 65+ had comorbid dementia and stroke in 2016-17 and were more likely to die (all causes of death) during the year than those without the comorbidity. With an aging population, comorbidities will be more prevalent, with impacts on health outcomes and related healthcare needs.

\section{Using the 'Confusion Assessment Method' (CAM) Tool to Screen for Delirium Among Hospitalized Inpatients-A Quality Improvement Initiative}

\section{A. Rahman, F. Carr. University of Alberta Hospital.}

Background: Delirium is an acute, fluctuating, confusional state, associated with significant mortality and morbidity amongst hospitalized patients. Currently, no standardized process exists for delirium screening by nursing staff. The CAM (confusion assessment method) is a validated delirium screening tool which has the potential to standardize and improve delirium screening by nursing staff.

Methods: This study was conducted on the ACE unit at the University of Alberta Hospital, Edmonton, from June to August 2018. 7 educational sessions were conducted with nursing staff, which included CAM training and practice scenarios. The CAM was implemented for delirium screening for all patients at the time of unit admission, then twice daily. Results were documented using the CAM tracking log. Weekly plan do study act (PDSA) cycles were conducted. Outcome, process and balancing measures were calculated from the pre/post intervention data and from the PDSA evaluations. 
Results: 139 patients were screened with the CAM. Total number of delirious patient were 23 and CAM screening identified 21 cases. Total number of falls was 9 . The financial costs associated with the study were minimal (\$210 in total).

Discussion: Admission CAM screening was consistently high (91\%). There was significant variation in how often the CAM was used and its documentation by nursing staff. CAM correctly identified delirium in 21 of 22 cases $(95 \%)$, and was associated with a decreased incidence of falls and use of physical and pharmacological restraints.

Conclusion: Delirium screening with the CAM by nursing staff was associated with a significant reduction in important clinical complications (falls and pharmacological and physical restraint use); however, long term data is required to determine its impact on delirium duration and length of stay.

\section{Identifying Learning Needs in Medical Assistance in Dying: From the Perspective of Internal Medicine Residents}

\section{K. Reich, J. Hui, A. Tan. University of Calgary.}

Background: Medical assistance in dying (MAiD) was legalized in Canada in 2016. In response, there have been 6,749 medically assisted deaths since legislative enactment to October 2018, of which $78 \%$ of these deaths were of patients 65 years or older. Integration of MAiD into the medical curricula is important to provide trainees with the skills to care for patients requesting assistance in dying. To date, there is no literature available to direct educational content in MAiD. Our study aimed to determine the learning needs in MAiD for Internal Medicine (IM) residents.

Methods: IM residents were recruited during an academic half day. Demographic information and data on prior teaching in MAiD was obtained. Residents completed three patient cases created to test knowledge in MAiD. This was followed by a seminar on MAiD content. An inductive thematic analysis was performed on responses to identify learner gaps in MAiD knowledge.

Results: Twenty residents participated (54\% response rate) of which 55\% were in their first year of training. Despite $40 \%$ of residents having received prior teaching in MAiD, 75\% reported to be "not very confident" in responding to a MAiD request. All residents wanted more education in MAiD incorporated into their training. A high level thematic analysis revealed "resident discomfort with discussing MAiD, lack of understanding of MAiD policy, and perceptions that MAiD is only an option when symptoms are controlled."

Discussion: IM residents have knowledge gaps in MAiD eligibility, policy and procedures, and understanding their role and obligations in the request process.

Conclusion: There is a need for more residency training in this specialized area of end-of-life care, especially given the ethical and emotional complexities of approaching these requests, which are predominately coming from our geriatric population.

Optimizing the Physical and Social Environment Within Hospitals to Improve Care for Hospitalized Patients with Dementia: A Systematic Review

C. Reich' ${ }^{1}$, H. Lyons ${ }^{2}$, J. Holroyd-Leduc ${ }^{1} .{ }^{1}$ University of Calgary; ${ }^{2}$ Queen's University.

Background: The environment in hospitals can play a significant role in the outcomes for patients with dementia. The purpose of this study was to review the published literature on interventions that modified the physical and/or social environment within hospitals to improve care for patients with dementia.

Methods: In this systematic review, our search strategy included electronic databases (MEDLINE, Embase and CINAHL) to identify all published English-language articles up to June 2019. PRISMA guidelines were followed. Two independent reviewers assessed citations and full-text articles. Articles were included based on the following criteria: patients with dementia/cognitive impairment, comparison of an intervention with control condition, and reported clinical or health systems outcomes. Quality was assessed using GRADE.

Results: A total of 9,887 studies were screened, of which 14 were included. Seven studies modified the physical environment such as single vs. multi bed wards, electronic sensor alarms, environmental cues, a garden, a steam foot spa, a purpose-built wing, and a specialist medical/mental health unit. The remaining studies evaluated specific programs (i.e. art, music, multi-sensory behavioral therapy, exercise, 'Dementia Companions' and 'Cognition Champions'). The majority of studies were low-very to low quality. Interventions found to be effective at reducing length of stay (LOS) were multi-bed wards and a ten-week music program. Falls rates decreased with electronic sensor alarms, a ten-week music program, and Dementia Companions. Medication administration decreased with a ten-week music program and 'Cognition Champions'.

Discussion: There is evidence to show environment modification reduces LOS, fall rates and medication administration. However, evidence is of low quality and high heterogeneity.

Conclusion: Further research is needed to determine the most effective interventions to optimize hospital environments for persons living with dementia.

\section{Illness Acuity has an Impact on the Relationship between Frailty and Mortality in Emergency Department Patients Referred to Internal Medicine}

K. Rockwood ${ }^{1}$, M. H. Pulok ${ }^{1}$, A. M. van der Valk', O. Theou ${ }^{I} .{ }^{I}$ Dalhousie University; ${ }^{2}$ Nova Scotia Health Authority. 


\section{BACSU: FAMILY PHYSICIANS AND DEMENTIA CARE}

Background: This study examined the role of illness acuity on the relationship between frailty and mortality among older Emergency Department (ED) patients referred to internal medicine.

Methods: Two frailty tools, embedded in a Comprehensive Geriatric Assessment (CGA) were used: the Clinical Frailty Scale (CFS) and a deficit accumulation frailty index (FICGA). The CFS recorded the pre-admission frailty state. The FI-CGA was constructed using 57 health-related items and measured the patients' frailty state at admission. Illness acuity at admission was assessed using the Canadian Triage and Acuity Scale (CTAS).

Results: We included data from 808 ED patients (mean age \pm SD $80.8 \pm 8.8,54.4 \%$ female) seen by one of us (KR). The mean FI-CGA score was $0.44 \pm 0.14$ and the CFS was $5.6 \pm 1.6$ with $38 \%$ patients classified as low acuity (CTAS: $1-2$ ). About $17 \%$ and $33 \%$ of the patients died within 30 days and six months of the CGA, respectively. Compared to well patients with low acuity, 30-day mortality risk was 22.5 times (95\% CI: 8.2-61.7) higher for severely frail patients with high acuity. When acuity was low, the risk for 30-day mortality was significant only among those with the highest frailty scores (CFS 7-9, FI-CGA>0.5). When acuity was high, even low frailty (CFS 5-6, FI-CGA 0.4-0.5) was associated with greater 30-day mortality. Highest risk patients (severely frail, high acuity) showed a one-year mortality hazard $=7.5(4.8-11.8)$; the HR for five-year mortality was 4.7 (3.5-6.5).

Discussion: When acuity was low, the risk was significant only with very high levels of frailty, whereas when acuity was high, even lower levels of frailty were associated with greater mortality risk.

Conclusion: Across levels of frailty, higher acuity increased mortality risk.

\section{Restricted Mean Survival Time of Older Adults with Severe Aortic Stenosis Foregoing Transcatheter Aortic Valve Replacement}

J. Rodighiero ${ }^{1}$, N. Piazza², G. Martucci ${ }^{2}$, M. Spaziano ${ }^{2}$, K. Lachapelle ${ }^{3}$, B. de Varennes ${ }^{3}, M-C$. Ouimet ${ }^{4}$, J. Afilalo ${ }^{5}$. ${ }^{1}$ Centre for Clinical Epidemiology, Jewish General Hospital; ${ }^{2}$ Division of Cardiology, McGill University Health Centre; ${ }^{3}$ Division of Cardiac Surgery, Mc Gill University Health Centre; ${ }^{4}$ Centre for Innovative Medicine, Mc Gill University Health Centre; ${ }^{5}$ Centre for Clinical Epidemiology, Jewish General Hospital; Centre for Innovative Medicine, McGill University Health Centre; Division of Cardiology, Jewish General Hospital, McGill University.

Background: Few studies have prospectively measured frailty as a reason for foregoing transcatheter aortic valve replacement (TAVR) in older adults with severe aortic stenosis (AS). This study sought to compare patients who either proceeded or did not proceed with TAVR, to better understand the prevalence of frailty and other reasons cited by clinicians and patients, and to determine the impact of these reasons on restricted mean survival time (RMST).

Methods: An analysis of the McGill Frailty Registry was conducted between 2014 and 2018 at the McGill structural valve clinic. Consecutive nonsurgical patients referred for TAVR underwent a multidisciplinary assessment including frailty. For medically managed patients, the primary reason for foregoing TAVR was determined and codified. Vital status at 1 year was analyzed using the RMST and Kaplan-Meier survival analyses.

Results: The study consisted of 373 patients with a mean age of 82.4 years, of which 233 proceeded with TAVR and 140 did not. The primary clinician-cited reason was: comorbidity burden in $34 \%$, frailty in $23 \%$, procedural feasibility and risks in $16 \%$, and mild or unrelated symptoms in $27 \%$. Compared to the TAVR group, RMST was reduced by 2.0 months in the no intervention group $(95 \%$ CI $1.2,2.7)$ and by 1.1 months in the balloon aortic valvuloplasty (BAV) group (95\% CI $-0.2,2.5)$. Survival in the BAV group was preserved up until 8 months post-procedure, after which time it declined to the level of the no intervention group.

Discussion: Patients with severe AS referred for TAVR may not graduate to undergo the procedure on the basis of comorbidity, frailty, procedural feasibility and risks, and mild or unrelated symptoms.

Conclusion: The best treatment decision is one that follows from multi-disciplinary assessment, including objective assessment of frailty.

\section{Are Administrative Actions Enough to Change Treatment Decisions? A Case in Point Regarding Medical Considerations for Long-Term Tube Feeding in Patients with End-Stage Dementia in Israel}

A. Sawicki ${ }^{1}$, T. Dwolatzky ${ }^{2}$, A. M. Clarfield ${ }^{3}{ }^{I}$ The Center for Multidisciplinary Research in Aging, Ben-Gurion University of the Negev, Israel; ${ }^{2}$ The Center for Multidisciplinary Research in Aging, Ben-Gurion University of the Negev, Israel \& Geriatric Unit, Rambam Health Care Campus, and the Ruth and Bruce Rappaport Faculty of Medicine, Technion - Israel Institute of Technology, Haifa, Israel; ${ }^{3}$ The Center for Multidisciplinary Research in Aging \& Medical School for International Health, Ben-Gurion University of the Negev, Israel.

Background: Oral assisted feeding is generally accepted as preferable to tube feeding in the long-term (LT) treatment of patients with advanced dementia. In Israel, a previous study (2005) indicated that a substantial proportion of patients with end-stage dementia were fed enterally, while feeding via nasogastric (NG) tube rather than Percutaneous Endoscopic Gastrostomy (PEG) was favored (ratio 2:1). This preference was attributed partly to 
administrative incentives. A decade ago, a Ministry of Health policy reform aimed to neutralize the administrative incentives favoring the use of NG over PEG, and guidelines addressing this issue were revised. Despite these reforms, a recent study found that enteral feeding, including by means of NG tubes, was still common in this population.

Methods: A questionnaire administered to all members of the Israeli Geriatrics Society (IGS) to investigate the general views and considerations regarding various aspects of enteral feeding (NG vs. PEG) in LT geriatric care wards for end-stage dementia patients (on a likert scale 1-4).

Results: 72 geriatricians (response rate of 18\%) answered the survey. PEG was viewed preferable to a NG tube in general (Mean=3, $\mathrm{SD}=1)$ and in terms of quality of life (Mean=3.1, $\mathrm{SD}=0.9$ ) but not in terms of complications (Mean $=1.8$, $\mathrm{SD}=0.8$ ). Most stated that their decisions were not purely medical (Mean=1.9, $\mathrm{SD}=0.9$ ). The strongest consensus was that the choice of treatment is taken together with the patient's primary caregiver (Mean=3.6, $\mathrm{SD}=0.7$ ), though physicians were not inclined to encourage choosing PEG over NG (Mean=2.5, $\mathrm{SD}=1$ ).

Discussion: There is not a strong inclination preferring a PEG over a NG tube for LT care among Israeli clinicians.

Conclusion: Although it was previously hypothesized that bureaucratic incentives perpetuated the misuse of NG tubes, this study suggests the presence of other underlying causes.

\section{Hospital Volunteer Programs for Older Adults: A Scoping Review to Inform Future Research}

C. B. Serapio ${ }^{1}$, N. Foster ${ }^{2}$, S. Sinha ${ }^{I} .{ }^{1}$ Faculty of Medicine, University of Toronto; ${ }^{2}$ Healthy Ageing and Geriatrics, Sinai Health System.

Background: Volunteer programs that focus on providing care to older adults have been implemented across hospitals. The purpose of the study is to assess the extent of the literature regarding volunteer programs for hospitalized older adults and identity knowledge gaps.

Methods: We searched PubMed, PsycInfo, AgeLine, Google Scholar, and reference lists for publications on volunteer programs targeting older adults in different hospital settings (acute care, emergency care, and rehabilitation) between June 2008 and June 2019. After screening, full-text reviews were conducted to determine eligibility for data extraction. A research matrix was created to compare data from articles deemed eligible. Themes were developed to summarize findings, namely, (i) impact of inpatient volunteer programs on older adults, (ii) role, identity and motivations of volunteers, (iii) staff-volunteer dynamics, and (iv) health system impact of volunteer programs.

Results: We found 1134 citations. After screening and full-text reviews, 22 papers were eligible for data extraction. Most of them used qualitative or mixed methods methodologies, and the majority looked at programs in acute care settings. Volunteer programs have been shown to positively impact nutrition, socialization, management of dementia and delirium, and mobility. Volunteers have varying motivations for volunteering such as career exploration and altruism. Staff generally view programs positively but may initially express reservations. Cost-benefit analyses suggest that they reduce cost.

Discussion: Only a small number of studies assessed the impact of their models on clinical outcomes (e.g. delirium rates and falls). There is lack of evidence to understand what features influence the program effectiveness, and how they compare across hospital settings.

Conclusion: There is growing evidence that demonstrates that hospital volunteer programs for older adults positively impact patient outcomes and experience, but more research needs to be done to understand what features determine their effectiveness.

The Impact of Volunteer Engagement on the Ageing Population: A Descriptive Analysis of the Maximizing Ageing Using Volunteer Engagement (MAUVE) Program at Sinai Health System

C. B. Serapio ${ }^{1}$, N. Foster ${ }^{2}$, S. Sinha ${ }^{3} .{ }^{1}$ Department of Medicine, University of Toronto; ${ }^{2}$ Healthy Ageing and Geriatrics, Sinai Health System, ${ }^{3}$ Healthy Ageing and Geriatrics, Sinai Health System; Department of Medicine, University of Toronto.

Background: MAUVE is a program that provides opportunities to hospital volunteers to engage with frail older adults at Sinai Health since 2010. It now operates across seven acute care units, two rehabilitation units, and the emergency department. The study's purpose is (i) to understand the medical complexity of MAUVE patients and interventions they receive and (ii) evaluate the impact of MAUVE on staff satisfaction.

Methods: A retrospective data analysis was conducted from patients that were visited by volunteers in the acute care units between Feb 2017 and June $2019(\mathrm{~N}=2,119)$ and in the rehab units between Oct 2018 and Feb 2019 (N=34). Descriptive statistics were generated that included demographics, average time spent by volunteers, and types of volunteer-led interventions performed. We also analyzed satisfaction surveys conducted between May and Aug 2019 from 66 acute care and 15 rehabilitation staff.

Results: A large percentage of MAUVE designated patients had $\mathrm{mood} / \mathrm{memory}$ issues (62\%), sensory impairments (49\%), were deemed at high risk of delirium (62\%) and nutritional decline $(21 \%)$, while $68 \%$ had inadequate social supports. Volunteers were found to spend more time with patients that were at high risk of nutritional decline and seldom visited by friends/family. Types of intervention being performed by volunteers appeared to be impacted by the patient's disease/ condition. According to surveyed staff, volunteers enhance 
care $(88 \%)$ and release time for them to provide more patient care $(75 \%)$.

Discussion: n/a.

Conclusion: Our study identified important findings that can guide future program implementation and research, especially around being able to better anticipate volunteer needs of older patients based on their characteristics and around the development of care plans for specific patient subpopulations of older adults. The current program delivers high staff satisfaction; this should be continuously monitored.

\section{An Ecological View: Grassroots Creation of a Dementia Friendly Community Club}

K. Shapkin ${ }^{1}$, G. McGhan ${ }^{2}$, L. Poole ${ }^{3}$, J. Power-Cyr ${ }^{4}$. ${ }^{1}$ University of Victoria; ${ }^{2}$ University of Calgary; ${ }^{3}$ Dementia Advocacy Canada; ${ }^{4}$ Glencoe Club.

Background: Currently 564,000 Canadians are living with dementia. Goodharzi and Ismail (Practical Neurology, 2019) report that $97 \%$ of people with dementia (PwD) experience neuropsychiatric symptoms (NPS) and apathy is the most common feature. Apathy is characterized by a lack of motivation to participate in activities and contributes to a decline in function. Family caregivers experience distress as they witness this change, knowing there is no pharmacotherapy treatment. A small group of caregivers motivated by these challenges created solutions at a sports and leisure club to include PwD in club activities.

Methods: The ecological theory of person-environment interaction proposed by Lawton and Nahemow's (The Psychology of Adult Development and Aging, 1973) was applied to provide a lens to gain an understanding of the club environment for PwD and their caregivers.

Results: The grassroots, family caregivers sought to change the club environment and reduce the pressure caused by a lack of a dementia programming. Strategically, club membership was surveyed (response rate $=58 \%$ ), and a high need to provide activities for PwD was identified. This overwhelming support resulted in dementia focused episodic education sessions, arts-based, cognitive and physical fitness programming and one-to-one peer support. In addition, the Alzheimer's Society, Best Friends program was incorporated $(80 \%$ staff completion rate).

Discussion: The environmental-press framework provides a view to see the psychological and physical capabilities of PwD when challenges in the environment are modified. The changes created opportunities for new interests and interconnections between PwD and the Club as a method of reducing apathy.

Conclusion: Recognizing the importance of the environment as a support for people with dementia and their families in the context of apathy is essential. Future plans include surveying the club membership again.

\section{Comparative Meta-Analytic Review of the Neuropsychological Profiles of ADHD in Adults and Late-Life and Mild Cognitive Impairment}

M. Sharma ${ }^{1}$, A. Plamondon ${ }^{2}$, B. Callahan ${ }^{1} .{ }^{1}$ University of Calgary; ${ }^{2}$ Université Laval.

Background: Although attention-deficit hyperactivity disorder (ADHD) can persist into later life, it is not well identified in older adults, partly due to overlapping features with mild cognitive impairment (MCI). This network meta-analysis compared the cognitive profiles of adults with ADHD or MCI, relative to age-matched controls.

Methods: We searched PubMed and PsycINFO for articles about cognition in adult ADHD (18-49 years; 25 articles retained), late-life ADHD (age $\geq 50 ; 2$ articles) or MCI (20 articles). Cognitive data were extracted and categorized into global cognition, attention, speed, verbal and visual memory, language, executive function (EF) and visuospatial domains. For each, mean effect sizes ( $\mathrm{d}$ ) were calculated within each group relative to controls using Cohen's d. Effect sizes were compared between groups based on $95 \%$ confidence intervals.

Results: Effect sizes for global cognition were greater in MCI $(\mathrm{d}=-1.289)$ than in ADHD $(\mathrm{d}=-0.340)$ and late-life ADHD ( $(\mathrm{d}=-0.222)$. Those for speed were greater in ADHD $(-\mathrm{d}=-2.096)$ than in MCI $(\mathrm{d}=-0.880)$ and late-life ADHD $(\mathrm{d}=0.188)$. Effect sizes for verbal memory were equal for ADHD ( $\mathrm{d}=-0.616)$ and MCI $(-\mathrm{d}=-1.743)$, and both greater than late-life ADHD ( $\mathrm{d}=-0.096)$. Similar effect sizes were found between ADHD and MCI for visual memory ( $\mathrm{d}=-$ 0.416 and $^{-} \mathrm{d}=-3.106$, respectively) and language $(\mathrm{d}=-1.181$ and ${ }^{-} \mathrm{d}=-0.872$, respectively) but could not be calculated for late-life ADHD due to insufficient data. EF effect sizes were equal for $\mathrm{ADHD}(-\mathrm{d}=-1.235)$ and $\mathrm{MCI}(-\mathrm{d}=-1.147)$, and both greater than late-life ADHD ( $\mathrm{d}=-0.308)$. Visuospatial effect sizes were greater for MCI $(-\mathrm{d}=-0.660)$ than ADHD $(-\mathrm{d}=-$ 0.083; data unavailable for late-life ADHD).

Discussion: Late-life ADHD and MCI both include features of impaired global cognition, memory and EF.

Conclusion: These features appear less marked in late-life ADHD, suggesting that cognitive symptoms of ADHD may improve with age.

\section{Perceptions of Physiatry Residents on Blended Learning in Their Geriatric Rehabilitation Education: A Qualitative Study}

A. Steiman ${ }^{l}$, A. Kam ${ }^{1}$, L. Sivarajah ${ }^{2}$, D. Richardson ${ }^{1}$.

${ }^{1}$ University Health Network/Toronto Rehab; ${ }^{2}$ University of Toronto Faculty of Medicine.

Background: Blended learning is defined as the thoughtful fusion of face-to-face and self-learning experiences and this approach takes cognitive load therapy and self-determination 
theory into consideration. While blended learning has been shown to reduce dropout, increase subject engagement and improve overall learning outcomes in undergraduate programs, there remains a relative lack of research examining post graduate residents' perception of blended learning. This study sought to gain insight into the understanding and perceptions of a specific subset of geriatric medicine learners, physiatry residents, regarding the potential benefits and limitations of blended learning during their geriatric rehabilitation rotation.

Methods: After a literature review and ethics approval, we sent out recruitment emails to all current physiatry residents and graduates (within the last 5 years). Reminder emails were sent out once a month in 5 consecutive months in order to maximize participation. For all interested participants, we conducted individual semi-structural interviews, through telephone or in-person. We then used a thematic analysis framework to analyze our data. Three separate script analysis were carried out for each interview.

Results: The participation rate was $18.4 \%$ ( 7 out of 38 subjects). Due to the homogeneous group, saturation on themes was noted among the participants. All 7 subjects have little understanding on blended learning, but they are receptive to this learning style.

Discussion: Based on the participant feedback, the benefits of implementing Blended Learning include active learning, adaptability to different styles, and adding concrete structure to the current curriculum. Participants indicate that the factors to consider prior to implementation are time constraints during a rotation, learners' motivation, and individual preferences.

Conclusion: Blended Learning may be a tool to enrich the geriatric rehabilitation curriculum for physiatry residents with potential to expand to all geriatric learners.

\section{The Issues Faced by Familial Caregivers of Persons with Dementia and Comorbid Depression, and Strategies to Better Support Them: A Scoping Review}

\author{
A. Subota, N. Spotswood, M. Roach, J. Holroyd-Leduc. \\ University of Calgary.
}

Background: Comorbid dementia and depression is associated with caregiver burden. Given family caregivers provide a significant proportion of care, they require support. A scoping review on issues faced by caregivers of individuals with dementia and depression, along with strategies to support them, was conducted.

Methods: MEDLINE, Embase, and PsycINFO were searched following the PRIMSA statements, and a grey literature search was done using CADTH Grey Matters. Our PCC was: Population $=$ Caregivers of persons with dementia and depression; Concept $=$ what issues do caregivers face and are there interventions to decrease caregiver burden and other adverse outcomes; Context $=$ any study design that focuses on family/friend caregivers. English language sources containing data on the effect of depression in persons with dementia on their caregivers were included. Two reviewers reviewed all citations and full-text articles. Data was extracted using a standardized abstraction form.

Results: 12,820 unique sources were identified; 93 studies were included. Study designs included cross-sectional, cohort, quasi-experimental, RCTs, and qualitative. Study size varied from $14-1249$ caregiver/care recipient dyads. Depression and dementia variability impacted a variety of caregiver outcomes: burden $(n=38)$, depression $(n=20)$, distress $(n=33)$, quality of life $(n=4)$, health and well-being $(n=8)$. Four studies of pharmacological interventions to improve depressive symptoms in persons with dementia found caregiver distress decreased but caregiver burden was unaffected. Seven non-pharmacological studies found caregiver distress and burden improved in some studies, but remained unchanged in others.

Discussion: Depression in dementia appears related to poorer caregiver outcomes. However, there are a variety of pharmacological and non-pharmacological interventions that could improve both depression in persons with dementia and caregiver burden.

Conclusion: Supports can improve outcomes for caregivers of persons with dementia and depression.

\section{Simulation Modeling for the Delivery and Scaling of a Specialized Geriatric Telemedicine Model of Care: A Case Study of Data-Driven Optimization GeriMedRisk}

H. T. Khajavi ${ }^{1}$, M. Pavlin ${ }^{2}$, J. Man-Wai Ho ${ }^{3} .{ }^{1}$ GeriMedRisk; ${ }^{2}$ Lazaridis School of Business and Economics, Wilfrid Laurier University; ${ }^{3}$ GeriMedRisk; Department of Medicine, McMaster University; Schlegel Research Institute for Aging.

Background: Canada has insufficient clinicians with expertise in geriatric medicine, clinical pharmacology, geriatric psychiatry and geriatric pharmacy. Designing and scaling a novel interdisciplinary telemedicine geriatric clinical pharmacology and psychiatry consult service to support older adults with complex physical and mental illness, such as GeriMedRisk (GMR), presents logistical challenges. These challenges include variable and growing demand, limited financial and human resources, and the need to keep wait-times within five days despite providing comprehensive interdisciplinary clinical recommendations. We conducted a set of simulations to determine the optimal team required to scale GMR from serving three regions to the entire province of Ontario, Canada.

Methods: Patient setting, consultation arrival process, service delivery and exit process are the main workflow components. Using historical GMR clinician consult and wait times from the regional service model, we developed a workflow simulation model with Simpy framework on Python. We studied 
the impact of demand, care setting and employee utilization rate on wait-times.

Results: We found the mean ratio of time spent per service for a consult was 2.4, and 1.1 for geriatric pharmacy, and geriatric clinical pharmacology, respectively, with geriatric psychiatry as reference. An on-call team comprised of 6 geriatric pharmacists, one geriatric psychiatrist; and one geriatrician or clinical pharmacologist was necessary to support the whole province while fulfilling the established five business day wait time.

Discussion: Simulation results showed that increasing the human resources bring about improvement in waiting times. However, increasing the number of pharmacists only may deteriorate the situation because a "bottleneck" is developed between the pharmacists and the psychiatrist.

Conclusion: Simulation models are efficient data-driven tools for justifying budget proposals of a growing complex geriatric telemedicine model of care for research grants or government funding.

\section{Frailty in Middle-Aged and Older Canadians}

O. Theou, M. U. Pérez-Zepeda, K. Rockwood. Dalhousie University.

Background: Frailty is a health care priority for aging countries, including Canada. The purpose of this project is to describe levels of frailty in Canadians $\geq 45$ years old, provide national normative data, and compare frailty levels across the Canadian provinces and levels of social vulnerability.

Methods: This is secondary analysis of the Canadian Longitudinal Study on Aging. After screening all available variables in the dataset, we constructed a 52-item Frailty Index (FI) and a 46-item Social Vulnerability Index (SVI) based on the deficit accumulation approach.

Results: We included data from 51,338 Canadians aged 45-85 years (mean age 60.3 years, $51.5 \%$ female). The mean FI score was $0.07 \pm 0.06$ and the mean SVI was $0.32 \pm 0.11$. The proportion of males with $\mathrm{FI} \leq 0.1,0.11-0.2,0.21-0.3$ and $\geq 0.31$ were $75.5 \%, 21.5 \%, 2.4 \%$ and $0.6 \%$, respectively. The proportion of females with FI $\leq 0.1,0.11-0.2,0.21-0.3$ and $\geq 0.31$ were $67.7 \%, 27.2 \%, 4.4 \%, 0.7 \%$, respectively. National normative data were identified for each year of age for males and females. New Brunswick had the highest FI (0.08 95\%CI 0.08-0.08), while British Columbia and Ontario (0.07 95\% CI 0.07-0.07) had the lowest. SVI was associated with lower FI scores.

Discussion: Levels of frailty are high even among middleaged Canadians. British Columbia and Ontario are the provinces with the lowest frailty burden and New Brunswick has the highest frailty burden. Social vulnerability could impact levels of frailty.

Conclusion: Examining frailty in the Canadian Longitudinal Study on Aging can inform discussions about frailty in Canada and set a new bar in the field.

\section{Examining the Relationship Between Antioxidant Brain Glutathione and White Matter Hyperintensities in Early Mild Vascular Cognitive Impairment}

M. T. Thiyagarajah, J. J. Chen, N. Herrmann, D. Gallagher, S. E. Black, F. Gao, J. Ramirez, S. Graham, P-S. Wei, P. I. Oh, A. C. Andreazza, A. Kiss, W. Swardfager, K. L. Lanctôt. University of Toronto.

Background: White matter changes are a hallmark of vascular cognitive impairment (VCI) and white matter hyperintensities $(\mathrm{WMH})$, as seen on magnetic resonance imaging, have been associated with cognitive decline. Oxidative stress markers have been associated with white matter lesions, with a proposed mechanism of higher inflammation and hypoperfusion leading to oxidative stress damaging DNA and lipids, which causes cell death and impairs renewal of glial cells. However, antioxidant mechanisms in $\mathrm{VCI}$ are unclear. We investigated the association between the endogenous antioxidant glutathione (GSH) in the brain and $\mathrm{WMH}$ in mild $\mathrm{VCI}(\mathrm{mVCI})$, an early stage of VCI.

Methods: Patients with mVCI aged 55 to 85 have been recruited from a cardiac rehabilitation program. The Fazekas scale was used to classify the extent of white matter lesion burden seen on neuroimaging. Brain GSH was quantified using proton magnetic resonance spectroscopy (MRS), specifically the Mescher-Garwood Point Resolved Spectroscopy (MEGA-PRESS) pulse sequence. The occipital lobe (OL) and anterior cingulate cortex (ACC) were selected as regions of interest due to their MRS signal quality and importance to mVCI relevant cognitive domains.

Results: To date, 10 patients ( $80 \%$ male, mean $\pm \mathrm{SD}$ age $=65 \pm 8$, Montreal Cognitive Assessment $=21 \pm 4$ ) have been enrolled. Preliminary results found that patients with Fazekas score 2 $(n=4)$ had higher levels of GSH in the OL than patients with Fazekas score $1(\mathrm{n}=6)$ as determined by one-way ANOVA $(\mathrm{F}(1,8)=6.240, \mathrm{p}=0.037)$. GSH in the ACC did not differ significantly between patients with Fazekas score 1 and 2 $(\mathrm{F}(1,8)=1.013, \mathrm{p}=0.345)$.

Discussion: Patients with greater extent of white matter damage may have higher levels of GSH, suggesting increases to compensate for OS in $\mathrm{mVCI}$.

Conclusion: Understanding the GSH system in mVCI may provide insight into potential therapeutic interventions.

\section{Exploring the Quality of Life of Patients with Shingles: A Qualitative Research Study}

D. Van Oorschot ${ }^{1}$, A. McGirr ${ }^{2}$, P. Goulet ${ }^{1}$, R. Chen ${ }^{3}$, R. Pratiwadi ${ }^{3}$, S. Shah ${ }^{3}$, S. Clifford ${ }^{3}$, D. Curran ${ }^{1} .{ }^{1} G S K$ Wavre; ${ }^{2}$ GSK Canada; ${ }^{3}$ ICON plc.

Background: Herpes Zoster (HZ) is caused by the reactivation of the varicella zoster virus, which causes chickenpox. HZ 
is characterized by a painful unilateral rash. About $10-30 \%$ of patients develop post-herpetic neuralgia ( $\mathrm{PHN})$, and approximately $10 \%$ experience ocular, cutaneous or neurological complications. Although the zoster brief pain inventory is frequently used to quantify pain and the impact of $\mathrm{HZ}$ on activities of daily living, few qualitative studies have been performed to better understand the disease and its impact from the patient's perspective. This qualitative study collects patient experiences aiming to describe the $\mathrm{HZ}$ burden of disease.

Methods: A hypothetical conceptual model was developed based on a targeted literature review to define concepts that are relevant for $\mathrm{HZ}$ patients. This model served as input for a semi-structured interview guide. Acute $\mathrm{HZ}$ patients (7-30 days after diagnosis), subacute $\mathrm{HZ}$ patients (31-60 days after diagnosis) and PHN patients (90-365 days after HZ diagnosis) were interviewed and transcripts were analyzed using an induction-abduction thematic analysis.

Results: Saturation of symptoms disclosed during interviews was reached after 12 subjects, with pain, rash, and fatigue as common symptoms. Impact on quality of life reached saturation after 8 interviews with emotional functioning, activities of daily living, sleep, hobbies, and physical functioning being the most commonly reported impacts. Pain and/or pain management was reported most frequently as the most challenging aspect of the disease. Excerpts describing the patient experience, stratified by HZ and PHN, will be presented.

Discussion: Understanding the patient journey can help healthcare professionals, decision-making bodies and patients' relatives understand the extent to which a patient's quality of life can be affected by HZ.

Conclusion: HZ can have a profound impact on a patient's quality of life.

\section{Canadian Older Adults' Perceptions and Behaviours Regarding Influenza Vaccines and Equity of Provincial Immunization Programs}

N. Waite ${ }^{1}$, M. Andrew ${ }^{2}$, V. Gilca ${ }^{3}$, S. Houle ${ }^{1}$, J. Pereira ${ }^{4}$.

${ }^{1}$ University of Waterloo; ${ }^{2}$ Dalhousie University; ${ }^{3}$ Laval

University; ${ }^{4}$ JRL Research \& Consulting Inc.

Background: Influenza has important health consequences in older adults. However, immunization rates are sub-optimal in Canadians $\geq 50$ years. We explored vaccine-related preferences and perceptions of access equity in this population.

Methods: In August 2019, we conducted an online survey of Canadian adults aged $\geq 50$ via a market research panel platform. Respondents aged 50-64 were asked about their satisfaction with the current influenza vaccine, and need for a more effective formulation. Those $65+$ were asked about the currently available regular-dose influenza vaccine and high-dose (HD) influenza vaccine, as the latter was publicly funded in a limited number of provinces during the 2018/19 influenza season.
Results: Of 1,006 respondents aged 50-64 years (mean age $=$ $57.4 ; 50.1 \%$ male), $488(48.5 \%)$ were satisfied with the current influenza vaccines, though 502 (49.9\%) would choose a more effective vaccine if free or $302(30 \%)$ if at a cost. If a more effective vaccine was free in other provinces but not in their own, $72.8 \%$ would be bothered; this includes $68.3 \%$ of the 559 respondents who were not vaccinated during the 2018/19 season. Of 3,548 adults $\geq 65$ (mean age $=71.4 ; 47.9 \%$ male), $621(17.5 \%)$ would opt for regular-dose influenza vaccine while $1,245(35.1 \%)$ selected HD vaccine if free. If HD was not free, $741(20.9 \%)$ would choose it while $871(24.5 \%)$ would opt for regular-dose vaccine, and 2,204 (62.2\%) would be bothered if it was free in other provinces.

Discussion: Satisfaction with current influenza vaccines is relatively low. Provincial inequity in access to free effective vaccine was bothersome to most respondents, regardless of their recent vaccination status.

Conclusion: Making effective influenza vaccines available through publicly funded provincial programs may positively impact immunization rates.

\section{Impact of Influenza on Canadian Adults Aged $\geq 50$ Years During the 2018/2019 Season}

\section{N. Waite ${ }^{1}$, M. Andrew ${ }^{2}$, V. Gilca ${ }^{3}$, S. Houle ${ }^{1}$, J. Pereira ${ }^{4}$. ${ }^{1}$ University of Waterloo; ${ }^{2}$ Dalhousie University; ${ }^{3}$ Laval University; ${ }^{4}$ JRL Research \& Consulting Inc.}

Background: Influenza has been associated with a decline in functional abilities and exacerbation of comorbidities among Canadian older adults, although its impact has not been fully examined.

Methods: In August 2019, we conducted a survey of Canadian adults aged $\geq 50$ through an online market research panel platform. The survey included questions about influenza experiences during the 2018/19 season, and the impact of diagnosed influenza or self-reported influenza-like-illness (ILI) on healthcare resource utilization, and ability to work, volunteer, and provide care to others.

Results: We surveyed 1006 adults aged 50-64 years (mean age $=57.4 ; 50.1 \%$ male; $48.1 \%$ with chronic conditions) and 3548 adults $\geq 65$ (mean age $=71.4 ; 47.9 \%$ male; $63.1 \%$ with chronic conditions). Of those 50-64 years, 230 (22.9\%) reported influenza/ILI, and $73(31.3 \%)$ took longer than 2 weeks to recover. During illness, 94 (40.9\%) and 57 (24.8\%) visited their pharmacy and family physician, respectively. Of the 143 respondents in the workforce, $74(51.7 \%)$ were absent due to influenza/ILI (mean $=4.2$ days); additionally, $79(55.2 \%)$ reported working at reduced capacity. Of the 97 individuals who were caregivers, $40(41.2 \%)$ were unable to do so while ill (mean $=5.3$ days). In the $\geq 65$ year age group, 501 (14.1\%) had influenza/ILI, of whom 187 (37.3\%) had illness lasting over 2 weeks, 188 (37.5\%) visited a pharmacy and 151 (30.1\%) saw their family physician. Of the $164(32.7 \%)$ 
individuals who volunteered, $80(48.8 \%)$ were unable to while ill (mean $=14.4$ hours $)$.

Discussion: Influenza and ILI among older Canadians have notable impacts on healthcare resource utilization, and ability to work, volunteer, and provide care to others.

Conclusion: Influenza prevention optimization in the older adult population would yield important societal benefits.

\section{Familial Association Between Attention-Deficit/ Hyperactivity Disorder and Neurocognitive Disorders \\ H. Weerawardhena, J. Milner, B. Callahan. University of Calgary.}

Background: Relative to healthy controls, individuals with attention-deficit/hyperactivity disorder (ADHD) are more likely to be diagnosed with Lewy body dementia (DLB) in old age, and persons with DLB are more likely to report childhood features of ADHD. Certain genetic variants have also been linked to both disorders, suggesting a shared disease process which may lead them to occur together in families at higher-than-chance rates. We hypothesize that individuals with ADHD will report more frequent occurrences of DLB in their families relative to other neurocognitive disorders and relative to controls without $\mathrm{ADHD}$.

Methods: Participants recruited through an online platform completed an online survey about the presence and onset of different neurocognitive disorders in all biological parents and grandparents. The Adult ADHD Self-Report Scale (ASRS) Part A was also used to determine the presence and severity of participants' own ADHD symptoms ( $\geq 14$ is considered clinically significant).

Results: The questionnaire was completed by 526 participants aged 17-66 years (22.2\% male). Thirty-six $(6.8 \%)$ self-reported a formal diagnosis of ADHD and obtained an average ASRS score of $16.11(\mathrm{SD}=3.77 ; \mathrm{t}=-8.736, \mathrm{p}<.001$ relative to non-ADHD participants, $\mathrm{M}=8.85, \mathrm{SD}=4.22)$. The ADHD and non-ADHD groups reported similar frequencies of Alzheimer's disease (19.4\% and $13.9 \%$, respectively; $\chi^{2}=2.25$, $\mathrm{p}=.325$ ), DLB (both $0.0 \% ; \chi^{2}=0.22, \mathrm{p}=.638$ ) and Parkinson's disease $\left(5.5 \%\right.$ and $\left.8.3 \% ; \chi^{2}=0.66, p=.719\right)$ in family members. The ADHD group reported higher frequencies of family members with mild cognitive impairment (MCI;13.9\%) than controls $\left(3.9 \% ; \chi^{2}=10.05, \mathrm{p}=.007\right)$.

Discussion: Contrary to the hypothesis, ADHD participants reported more frequent occurrences of MCI, but not DLB, in their families relative to other neurocognitive disorders and relative to non-ADHD controls.

Conclusion: Given the heritability of ADHD, this finding could be due to manifestation of MCI-like ADHD symptoms in older adults.
GERIOSCOPE. GERiatric cOhort aSsessment in Intensive Care Unit: OPportunities and fEasibility

T. Wissanji ${ }^{1}$, D. Beaudin ${ }^{2}$, M-F. Forget ${ }^{3}$, C. Faubert ${ }^{4}$, H. T. Wang 4 . ${ }^{1}$ Hôpital Sacré-Coeur de Montréal; ${ }^{2}$ Centre Hospitalier de l'Université de Montréal; ${ }^{3}$ Université de Montréal; ${ }^{4}$ Hôpital Maisonneuve-Rosemont.

Background: Geriatric speciality and Comprehensive Geriatric Assessment (CGA) have improved older adult outcomes in emergency, perioperative and oncologic settings. Critically ill older adults might also benefit from it. But foreseeable obstacles need to be addressed to envisioning geriatric assessment in the critical care setting. Our aim was to study feasibility of geriatric consultation in an intensive care unit (ICU).

Methods: We performed a retrospective observational study of adults aged 70 years and above admitted in our ICU between July 29, 2019, and August 16, 2019. Patients underwent a geriatric consultation from a fellow in geriatrics, supervised by a geriatrician and an intensivist. Geriatric consultations were systematic for every older adult aged 70 and above or upon ICU team request. Admissions for elective surgery were excluded. Data on CGA, extracted from the geriatric assessment, and geriatric recommendations were found in charts. Results were reported with descriptive statistics.

Results: From the ICU admissions, 13 patients were evaluated by the dedicated geriatric team (11 eligible patients, 2 patients with geriatric profiles on treating team request). Median age was 79 (73-83, range 69-95). The CGA was performed within 7 days of ICU admission for 11 (85\%) patients (median 2.45 (0-3)). On average, $71 \%$ of the items were completed. An average of 3.8 (1-5) suggestions were emitted per patients. The most frequent recommendation concerned advance care preference $(10,75 \%)$. Almost all emitted recommendations $(43 / 49,90 \%)$ were accepted by the treating physician.

Discussion: Because of the critical nature of the patients' condition, completing the CGA was challenging. Geriatric consultation was complementary in prognostication and proposed interventions.

Conclusion: Our study suggests that CGA can be performed in critical care and leads to concrete recommendations that were broadly accepted by the treating physician.

\section{Online Medication Management Educational Program for Caregivers of People Living with Dementia}

S. Yau, S. Chernin, L. Sokoloff, R. Sham, S. Moy, A. Santiago, K. Nemethy, A. Shnall. Baycrest.

Background: Persons with dementia (PWD) often need help managing their medications and require support from their caregivers. Lack of proper medication management may result in early admission to long-term care facilities. To 


\section{BACSU: FAMILY PHYSICIANS AND DEMENTIA CARE}

address these concerns, we developed a caregiver-focused online educational program. The purpose of this study was to investigate the impact of the online educational module about medication management for PWD.

Methods: In this pilot study, caregivers $(\mathrm{n}=12)$ completed an online educational module that was informed by an initial needs assessment. The module included topics regarding managing medications for PWD. Caregivers completed questionnaires regarding satisfaction and self-efficacy at three time points: a) before; b) immediately after module completion; and c) three weeks post module completion.

Results: The majority of caregivers were female (67\%), completed post-secondary education/higher $(75 \%)$, and were employed (67\%). Most caregivers were spouses $(42 \%)$ of the PWD, did not $(75 \%)$ have specific training in caring for PWD, and on average, have been providing care for 3.7 years $(\mathrm{SD}=3.0)$.

Discussion: After completing the online education module, caregivers reported: a) improvement in preparedness in finding and using information and services to help manage medications, b) increased awareness of medication side effects, and c) improvement in self-reported confidence in their ability to manage medications for PWD. Participant logs $(\mathrm{n}=10)$ indicated that caregivers spent between 1.5 to 3 hours on the online module and accessed $72 \%$ of the module, on average. Participants reported that the online module was "easy to access", "easy to navigate", and "easy to understand". Over $80 \%$ of caregivers would recommend the online module to PWD caregivers.

Conclusion: The online medication management education program for caregivers of PWD is a valuable tool for offering education for caregivers to build confidence in managing medications.

\section{Revised Canadian Geriatrics Society Medical Student Core Competencies in the Care of Older Persons 2020: Delphi Survey 1-Validating the Building Blocks}

T. Yogaparan ${ }^{1}, K . \mathrm{Ng}^{2}$, E. MacDonald ${ }^{3}$, M. Moran ${ }^{4}$, C. A. Sadowski ${ }^{4}$, J. Thain ${ }^{5}$, D. Mangat ${ }^{6}$, C. Talbot-Hamon ${ }^{7}$,
L. Khoury ${ }^{8}$, J. Smallbone ${ }^{9}$, C. Grief ${ }^{1}$, A. Burrell, S. Feldman, T. Bach, S. Lustgarten, S. Rai, A. Vickneswaran. ${ }^{1}$ Baycrest; ${ }^{2}$ Saini Health System; ${ }^{3}$ HorizonNB; ${ }^{4}$ Alberta Health; ${ }^{5}$ LHSC; ${ }^{6}$ University of Manitoba; ${ }^{7} \mathrm{Mc}$ Gill.ca; ${ }^{8} \mathrm{TOH} ;{ }^{9}$ University of Toronto.

Background: As the older adult population continues to increase dramatically, most physicians should be trained to meet the unique needs of older adults. In 2009, the Canadian Geriatrics Society (CGS) published their Core Competencies in the Care of Older Persons for Canadian Medical Students, listing 20 core competencies under 9 major headings. Considering the new CGS Geriatric 5M framework and gaps identified in the 2009 competencies, in 2019 the CGS Education Committee initiated a revision of the existing competencies.

Methods: A 14-member steering group was formed, and a literature search was conducted. The steering committee members identified gaps in the existing CGS medical student competencies. A modified Delphi process was initiated to reach consensus on the final competencies. For the first round of the modified Delphi process, existing competencies were broken down and listed along with identified gaps under the categories of skills, knowledge and attitudes, using the $5 \mathrm{M}$ framework. Participants were asked to rate the importance of, and expected skill or knowledge level for each component on a Likert scale.

Results: There were 64 participants nationwide. Sixty-seven percent of respondents were geriatricians, and 70 percent were in Ontario. There was overwhelming agreement on the relative importance of proposed components. Advocacy and communication skills were emphasized in the qualitative comments. In terms of knowledge level, most respondents expected students to at least understand the proposed component, although there was considerable variation in the expected skill level.

Discussion: As there was overwhelming agreement on the importance of the components of the competencies, we will proceed to the second round of the modified Delphi process.

Conclusion: Where participants will be asked to rate their agreement with specific proposed competencies. 